Modelling, Analysis and Simulation

MAS Numerical aspects of special functions

N.M. Temme

RePORt MAS-E0711 JuLY 2007 
Centrum voor Wiskunde en Informatica (CWI) is the national research institute for Mathematics and Computer Science. It is sponsored by the Netherlands Organisation for Scientific Research (NWO).

CWI is a founding member of ERCIM, the European Research Consortium for Informatics and Mathematics.

CWI's research has a theme-oriented structure and is grouped into four clusters. Listed below are the names of the clusters and in parentheses their acronyms.

Probability, Networks and Algorithms (PNA)

Software Engineering (SEN)

\section{Modelling, Analysis and Simulation (MAS)}

Information Systems (INS)

Copyright (C) 2007, Stichting Centrum voor Wiskunde en Informatica

P.O. Box 94079, 1090 GB Amsterdam (NL)

Kruislaan 413, 1098 SJ Amsterdam (NL)

Telephone +31205929333

Telefax +31205924199

ISSN 1386-3703 


\title{
Numerical aspects of special functions
}

\begin{abstract}
This paper describes methods that are important for the numerical evaluation of certain functions that frequently occur in applied mathematics, physics and mathematical statistics. This includes what we consider to be the basic methods, such as recurrence relations, series expansions (both convergent and asymptotic), and numerical quadrature. Several other methods are available and some of these will be discussed in less detail. Examples will be given on the use of special functions in certain problems from mathematical physics and mathematical statistics (integrals and series with special functions).
\end{abstract}

2000 Mathematics Subject Classification: 65D20

Keywords and Phrases: Computation of special functions

Note: Acta Numerica (2007), pp. 379--478. 



\title{
Numerical aspects of special functions
}

\author{
Nico M. Temme \\ Centrum voor Wiskunde en Informatica (CWI) \\ Kruislaan 413, NL-1098 SJ Amsterdam, \\ The Netherlands \\ E-mail: Nico.Temme@cwi.nl
}

\begin{abstract}
This paper describes methods that are important for the numerical evaluation of certain functions that frequently occur in applied mathematics, physics and mathematical statistics. This includes what we consider to be the basic methods, such as recurrence relations, series expansions (both convergent and asymptotic), and numerical quadrature. Several other methods are available and some of these will be discussed in less detail. Examples will be given on the use of special functions in certain problems from mathematical physics and mathematical statistics (integrals and series with special functions).
\end{abstract}

\section{CONTENTS}

$\begin{array}{llr}1 & \text { Introduction } & 1 \\ 2 & \text { Series expansions } & 3 \\ 3 & \text { Numerical quadrature } & 15 \\ 4 & \text { Recurrence relations } & 44 \\ 5 & \text { Uniform asymptotic expansions } & 58 \\ 6 & \text { The inversion of cumulative distribution functions } & 78 \\ 7 & \text { How to handle series with special functions } & 89 \\ 8 & \text { Software for computing special functions } & 96 \\ 9 & \text { Concluding remarks } & 97 \\ \text { References } & 97\end{array}$

\section{Introduction}

Special functions arise in various branches of applied mathematics, mathematical statistics, physics, and engineering in the form of integrals, as solutions of differential or difference equations, as integrands of integrals, as 
terms of infinite series, and so on. In which form they arise is not very important for their numerical evaluation, because for all the common special functions many analytic representations exist.

In this paper we discuss elements of a selection of methods that we consider to be most important for designing algorithms for special functions. Series expansions (convergent or asymptotic) are important, but usually the parameter domain cannot be completely covered by of convergent or asymptotic series. For the intermediate area we consider numerical quadrature and recurrence relations to be the most useful tools.

We start the paper with hypergeometric series, mainly for notational purposes, in particular the Gauss hypergeometric function ${ }_{2} F_{1}(a, b ; c ; z)$. We give information on the use of the several power series representations of this function in the complex $z$-plane, and give an alternative power series for domains that cannot be reached when using power series for the Gauss function.

The second basic method is numerical quadrature and we give an overview of the simplest, but also most efficient, quadrature rule for special functions, namely the trapezoidal rule. We consider this rule for both finite and infinite intervals, and present the remainders of this rule in several forms. Because the functions representing the integrands are always analytic in this area, we can apply the powerful results of complex analysis. The effectiveness of the rule and the elegant form of the remainders are mainly based on Cauchy's integral for analytic functions,

$$
f(z)=\frac{1}{2 \pi \mathrm{i}} \int_{\mathcal{C}} \frac{f(\zeta)}{\zeta-z} \mathrm{~d} \zeta
$$

where $\mathcal{C}$ is a circle around the point $z$ inside the domain where $f$ is analytic. Viewing this from the perspective of numerical quadrature, with some imagination, we can say that Cauchy's integral gives the relation between an integral and just one function value, and this rule is exact.

Recurrence relations form the third important tool. We give the theory that is of practical use for computing special functions, for example, the Miller algorithm. We give details for Bessel functions, Legendre functions, and Gauss hypergeometric functions.

In the remaining sections we give information on how to deal with socalled uniform asymptotic expansions, in which the coefficients are usually difficult to handle numerically. We use uniform asymptotic expansions for the incomplete gamma function for the asymptotic and numerical inversion of these functions for large parameters. In this way we describe a method for the inversion of cumulative distribution functions, which is an important topic in mathematical statistics.

In a final section we discuss two problems in which special functions play 
a role in series expansions; a distribution function and the solution of a singular perturbation problem.

Our approach in almost all discussions is to keep an eye out for the largeparameter case. Many published algorithms work fine for small- or mediumsized parameters. There are big challenges in extending existing algorithms, or developing completely new algorithms, for large real or complex parameters for rather common special functions. A second aspect of our approach is to consider the stability of the numerical method as the main issue. In particular, when several real or complex parameters occur, a strict error analysis is far beyond the daily practice of efficient numerical algorithms.

Almost all functions considered in this paper are defined in the Handbook of Mathematical functions, (Abramowitz and Stegun 1964), which has now been updated to form the Digital Library of Mathematical Functions, of which the book and web version will be published in the very near future; see also http://dlmf.nist.gov/.

For further properties of special functions we refer to (Olver 1997), where, just as in (Wong 2001), information on asymptotic analysis can be found. For an introduction to special functions, and for details of some examples considered in this paper, we refer to (Temme 1996). For a web link with many definitions and descriptions of special functions, as well as for generalized functions such as the Fox H-function, the Meijer G-function, the Kampé de Fériet function, the MacRobert E-Function, and the Appell functions, we refer to http://mathworld.wolfram.com/ .

The topics mentioned in this paper, and several other topics, will be discussed extensively, with examples of software, in a new book entitled Numerical Methods for Special Functions, written by Amparo Gil, Javier Segura, and the present author. This book will be published by SIAM in 2007.

\section{Series expansions}

Many special functions can be defined by power series that are of hypergeometric type. That is, they can be defined by power series of the form

$$
f(z)=\sum_{n=0}^{\infty} c_{n} z^{n},
$$

where $c_{n+1} / c_{n}$ is a rational function of $n$. Examples are

$$
e^{z}=\sum_{n=0}^{\infty} \frac{z^{n}}{n !}, \quad(1+z)^{a}=\sum_{n=0}^{\infty}\left(\begin{array}{l}
a \\
n
\end{array}\right) z^{n} .
$$


A useful framework for working with these functions is the class of generalized hypergeometric functions. We define

$$
{ }_{p} F_{q}\left(\begin{array}{c}
a_{1}, \cdots, a_{p} \\
b_{1}, \cdots, b_{q}
\end{array} z\right)=\sum_{n=0}^{\infty} \frac{\left(a_{1}\right)_{n} \cdots\left(a_{p}\right)_{n}}{\left(b_{1}\right)_{n} \cdots\left(b_{q}\right)_{n}} \frac{z^{n}}{n !},
$$

where $(a)_{n}$ is the Pochhammer symbol, also called the shifted factorial, defined by

$$
(a)_{0}=1, \quad(a)_{n}=a(a+1) \cdots(a+n-1) \quad(n \geq 1) .
$$

In terms of the gamma function we have

$$
(a)_{n}=\frac{\Gamma(a+n)}{\Gamma(a)}, \quad n=0,1,2, \ldots .
$$

The series in (2.3) defines an entire function in $z$ if $p \leq q$.

In the case $p=q+1$ the infinite series converges if $|z|<1$, and defines an analytic function in this disk. This function can be continued analytically outside the disk, with a branch cut from 1 to $+\infty$. Let

$$
\gamma_{q}=\left(b_{1}+\cdots+b_{q}\right)-\left(a_{1}+\cdots+a_{q+1}\right) .
$$

Then on the circle $|z|=1$ the series (2.3) is absolutely convergent if $\operatorname{Re} \gamma_{q}>$ 0 , convergent except at $z=1$ if $-1<\operatorname{Re} \gamma_{q} \leq 0$, and divergent if $\operatorname{Re} \gamma_{q} \leq-1$.

The binomial coefficient in (2.2) can be written in several forms,

$$
\left(\begin{array}{l}
a \\
n
\end{array}\right)=\frac{\Gamma(a+1)}{n ! \Gamma(a+1-n)}=(-1)^{n} \frac{\Gamma(n-a)}{n ! \Gamma(-a)}=(-1)^{n} \frac{(-a)_{n}}{n !},
$$

and we find that the series in (2.2) can be written as

$$
e^{z}={ }_{0} F_{0}\left(\begin{array}{l}
- \\
-
\end{array} z\right), \quad(1+z)^{a}={ }_{2} F_{1}\left(\begin{array}{c}
-a,- \\
-
\end{array}-z\right)={ }_{1} F_{0}\left(\begin{array}{l}
-a \\
-
\end{array} ;-z\right) .
$$

The second relation holds for $|z|<1$. When $a=m$, a non-negative integer, the binomial function in (2.2) becomes Newton's binomial formula, with only $m+1$ terms. Also from (2.7) we see that $(-m)_{n}$ equals 0 when $n \geq m+1$. In general, the power series in (2.3) terminates when one of the $a_{j}$ equals a non-positive integer. In that case $p$ and $q$ can be any non-negative integer.

On the other hand, the ${ }_{p} F_{q}$-function of (2.3) is not defined if one of the $b_{j}$ equals a non-positive integer, except in the following typical case. Let $a_{j}=-m$ and $b_{j}=-m-\ell$, with $\ell, m$ nonnegative integers. Then we have (cf. (2.7))

$$
\frac{\left(a_{j}\right)_{n}}{\left(b_{j}\right)_{n}}=\frac{(-m)_{n}}{(-m-\ell)_{n}}= \begin{cases}\frac{m !}{(m-n) !} \frac{(m+\ell-n) !}{(m+\ell) !}, & m \geq n \\ 0, & m<n\end{cases}
$$


Other examples of hypergeometric functions are the Bessel functions, with the special case

$$
\Gamma(\nu+1)\left(\frac{1}{2} z\right)^{-\nu} J_{\nu}(z)={ }_{0} F_{1}\left(\begin{array}{c}
- \\
\nu+1
\end{array} ;-\frac{1}{4} z^{2}\right)=e^{-\mathrm{i} z}{ }_{1} F_{1}\left(\begin{array}{c}
\nu+\frac{1}{2} \\
2 \nu+1
\end{array} ; 2 \mathrm{i} z\right),
$$

where $J_{\nu}$ denotes the ordinary Bessel function of the first kind.

The ${ }_{1} F_{1}$-function is also denoted by

$$
M(a, c, z)={ }_{1} F_{1}\left(\begin{array}{l}
a \\
c
\end{array} ; z\right),
$$

and $M$ is also called the confluent hypergeometric function. This entire function is a solution of the Kummer differential equation

$$
z w^{\prime \prime}+(c-z) w^{\prime}-a w=0 .
$$

A second solution of this equation is denoted by $U(a ; c ; z)$ and can be defined by

$$
U(a ; c ; z)=\frac{1}{\Gamma(a)} \int_{0}^{\infty} e^{-z t} t^{a-1}(1+t)^{c-a-1} \mathrm{~d} t .
$$

This function cannot be written as a convergent ${ }_{p} F_{q}$-function. However, see (2.18).

The Airy function $\operatorname{Ai}(z)$, a solution of the differential equation $w^{\prime \prime}-z w=$ 0 , is an entire function with power series representation

$$
\begin{aligned}
& \operatorname{Ai}(z)=c_{1} f(z)-c_{2} g(z), \\
& f(z)=1+\frac{1}{3 !} z^{3}+\frac{1 \cdot 4}{6 !} z^{6}+\frac{1 \cdot 4 \cdot 7}{9 !} z^{9}+\cdots, \\
& g(z)=z+\frac{2}{4 !} z^{4}+\frac{2 \cdot 5}{7 !} z^{7}+\frac{2 \cdot 5 \cdot 8}{10 !} z^{10}+\cdots, \\
& c_{1}=3^{-\frac{2}{3}} \Gamma\left(\frac{2}{3}\right), \quad c_{2}=3^{-\frac{1}{3}} \Gamma\left(\frac{1}{3}\right) .
\end{aligned}
$$

The functions $f$ and $g$ can be written as

$$
f(z)={ }_{0} F_{1}\left({ }_{\frac{2}{3}}^{-} ; \frac{1}{9} z^{3}\right), \quad g(z)=z_{0} F_{1}\left(\frac{-}{3} ; \frac{1}{9} z^{3}\right) .
$$

Remark 2.1. For large complex values with $|\mathrm{ph} z|<\pi$, the Airy function behaves as follows:

$$
\operatorname{Ai}(z) \sim \frac{1}{2} \pi^{-\frac{1}{2}} z^{-\frac{1}{4}} e^{-\frac{2}{3} z^{\frac{3}{2}}} .
$$

We have $\operatorname{Ai}(5)=0.000108344 \ldots$. This value cannot be obtained by using the representation of $\operatorname{Ai}(z)$ in the first line of (2.14) and the power series 
for $f$ and $g$ working to 10 digits. This is a nice example in which the power series, although convergent for all $z \in \mathbb{C}$, have a limited range of validity in numerical algorithms.

\section{Notation for asymptotic expansions}

When $\gamma_{q}$ of (2.6) satisfies $\gamma_{q} \leq-1$ the definition in (2.3) has no meaning because the series diverges. However, the notation may be used in an asymptotic sense. For example, the confluent hypergeometric function $U(a ; c ; z)$ defined in (2.13) has the large $z$ asymptotic expansion

$$
U(a ; c ; z) \sim z^{-a} \sum_{n=0}^{\infty} \frac{(a)_{n}(1+a-c)_{n}}{n !}(-z)^{-n}, \quad|\operatorname{ph} z|<\frac{3}{2} \pi,
$$

which we can write as

$$
U(a ; c ; z) \sim z^{-a}{ }_{2} F_{0}\left(\begin{array}{c}
a, 1+a-c \\
-
\end{array}-\frac{1}{z}\right) .
$$

This cannot be interpreted as an identity.

Remark 2.2. It is confusing that Maple 9.5 identifies the hypergeometric function in (2.18) with the Kummer $U$-function. For example, the Maple function Kummer $U$ gives the same numerical output as an evaluation based on the hypergeometric function shown on the right-hand side of (2.18), even when $z$ is small.

\subsection{The Gauss hypergeometric function}

The Gauss hypergeometric function is the case $p=2, q=1$, that is,

$$
{ }_{2} F_{1}\left(\begin{array}{c}
a, b \\
c
\end{array} ; z\right)=\sum_{n=0}^{\infty} \frac{(a)_{n}(b)_{n}}{(c)_{n} n !} z^{n}=1+\frac{a b}{c 1 !} z+\frac{a(a+1) b(b+1)}{c(c+1) 2 !} z^{2}+\cdots,
$$

where $c \neq 0,-1,-2, \ldots$ and $|z|<1$. It is a solution of the hypergeometric differential equation

$$
z(1-z) w^{\prime \prime}+[c-(a+b+1) z] w^{\prime}-a b w=0 .
$$

It is not difficult to verify that

$$
z^{1-c_{2} F_{1}}\left(\begin{array}{c}
a-c+1, b-c+1 \\
2-c
\end{array} ; z\right)
$$

is a second solution of $(2.20)$. 
If $\operatorname{Re}(c-a-b)>0$, the value of the Gauss hypergeometric function at $z=1$ is given by

$$
{ }_{2} F_{1}\left(\begin{array}{c}
a, b \\
c
\end{array} ; 1\right)=\frac{\Gamma(c) \Gamma(c-a-b)}{\Gamma(c-a) \Gamma(c-b)} .
$$

The power series for the Gauss hypergeometric function provides a simple and efficient means for the computation of this function, when $z$ is properly inside the unit disk. The terms can easily be computed by the recursion in the representation

$$
{ }_{2} F_{1}\left(\begin{array}{c}
a, b \\
c
\end{array} ; z\right)=\sum_{n=0}^{\infty} T_{n}, \quad T_{n+1}=\frac{(a+n)(b+n)}{(c+n)(n+1)} T_{n}, \quad n \geq 0, \quad T_{0}=1 .
$$

Other power series for the Gauss hypergeometric function

The power series in (2.19) converges inside the unit disk and for numerical computations we can use only the disk $|z| \leq \rho<1$. Other power series are available, however, to extend this domain.

The ${ }_{2} F_{1}$-function with argument $z$ can be written in terms of one or two other ${ }_{2} F_{1}$-functions with argument

$$
\frac{1}{z}, \quad 1-z, \quad \frac{1}{1-z}, \quad \frac{z}{z-1}, \quad \frac{z-1}{z} .
$$

For a useful set of relations we refer to (Abramowitz and Stegun 1964, p. 559) and (Temme 1996, pp. 110 and 113).

When we restrict the absolute values of the quantities in (2.24) to the bound $\rho$, with again $0<\rho<1$, we find, writing $z=x+i y$,

$$
\begin{array}{lll}
|z| \leq \rho, & \Longrightarrow & x^{2}+y^{2} \leq \rho^{2} ; \\
\left|\frac{1}{z}\right| \leq \rho, & \Longrightarrow & x^{2}+y^{2} \geq \frac{1}{\rho^{2}} ; \\
|1-z| \leq \rho, & \Longrightarrow & (x-1)^{2}+y^{2} \leq \rho^{2} ; \\
\frac{1}{|1-z|} \leq \rho, & \Longrightarrow & (x-1)^{2}+y^{2} \geq \frac{1}{\rho^{2}} ; \\
\left|\frac{z}{1-z}\right| \leq \rho, & \Longrightarrow & \left(x-\frac{\rho^{2}}{1-\rho^{2}}\right)^{2}+y^{2} \leq \frac{\rho^{2}}{\left(1-\rho^{2}\right)^{2}} ; \\
\left|\frac{z}{1-z}\right| \geq \frac{1}{\rho}, & \Longrightarrow & \left(x-\frac{1}{1-\rho^{2}}\right)^{2}+y^{2} \geq \frac{\rho^{2}}{\left(1-\rho^{2}\right)^{2}} .
\end{array}
$$

The domains defined by these inequalities do not cover the entire $z$-plane. The points $z=e^{ \pm \pi i / 3}$ do not satisfy these six conditions, for any $\rho \in(0,1)$. 

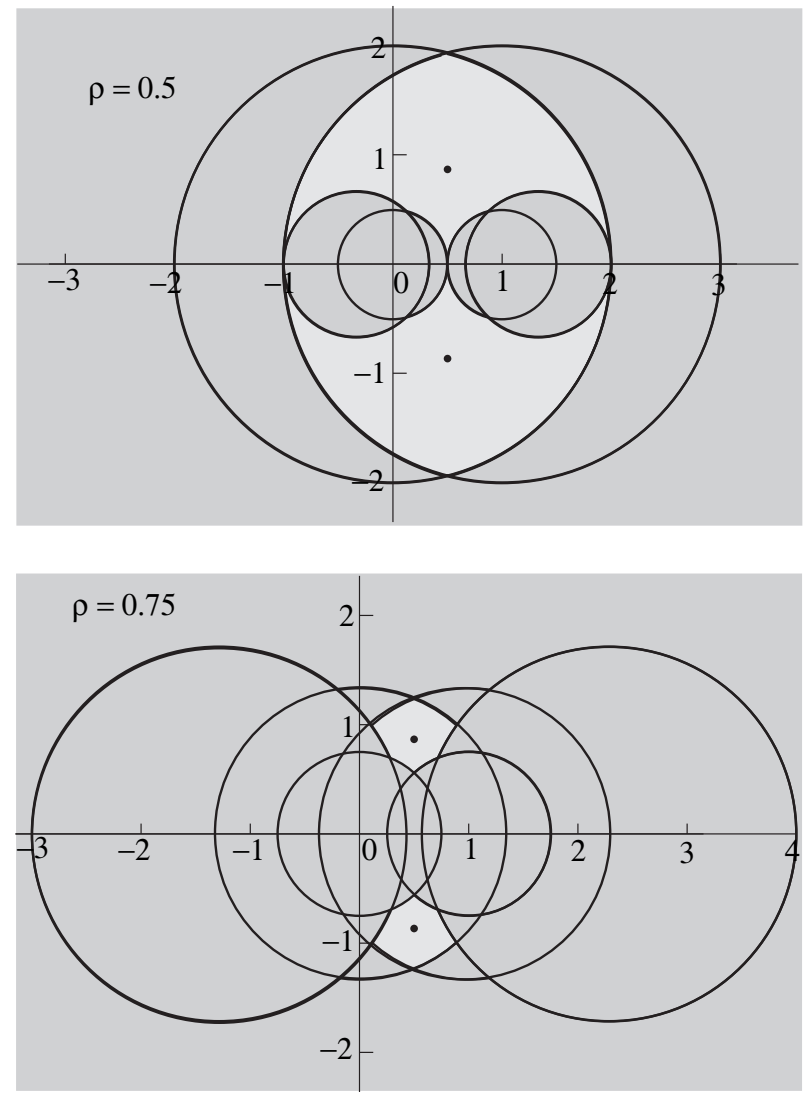

Figure 2.1. In the light domains none of the inequalities of (2.25) are satisfied. For $\rho \rightarrow 1$ these domains shrink to the points $e^{ \pm \pi i / 3}$, which are indicated by black dots.

When $\rho \rightarrow 1$, the domain of points not satisfying the six conditions shrinks to the exceptional points $z=e^{ \pm \pi i / 3}$. See Figure 2.1, where these points are indicated with black dots, for the cases $\rho=\frac{1}{2}$ and $\rho=\frac{3}{4}$. In the light area none of the inequalities of $(2.25)$ hold.

To compute the ${ }_{2} F_{1}$-functions in a neighbourhood of the points $z=e^{ \pm \pi i / 3}$ many other methods are available. One very useful method is discussed now.

\section{Bühring's analytic continuation formula}

I(Bühring 1987) derived power series expansions of the Gauss function, which enable computations near these special points. Bühring's expansion reads as follows. If $b-a$ is not an integer, we have for $\left|\operatorname{ph}\left(z_{0}-z\right)\right|<\pi$ the 
continuation formula

$$
\begin{aligned}
& { }_{2} F_{1}\left(\begin{array}{c}
a, b \\
c
\end{array} ; z\right)=\frac{\Gamma(c) \Gamma(b-a)}{\Gamma(b) \Gamma(c-a)}\left(z_{0}-z\right)^{-a} \sum_{n=0}^{\infty} d_{n}\left(a, z_{0}\right)\left(z-z_{0}\right)^{-n}+ \\
& \frac{\Gamma(c) \Gamma(a-b)}{\Gamma(a) \Gamma(c-b)}\left(z_{0}-z\right)^{-b} \sum_{n=0}^{\infty} d_{n}\left(b, z_{0}\right)\left(z-z_{0}\right)^{-n},
\end{aligned}
$$

where both series converge outside the circle $\left|z-z_{0}\right|=\max \left(\left|z_{0}\right|,\left|z_{0}-1\right|\right)$ and the coefficients are given by the three-term recurrence relation

$$
d_{n}\left(s, z_{0}\right)=\frac{n+s-1}{n(n+2 s-a-b)}\left[P_{n} d_{n-1}\left(s, z_{0}\right)+Q_{n} d_{n-2}\left(s, z_{0}\right)\right],
$$

where $n=1,2,3, \ldots$ and

$$
P_{n}=(n+s)\left(1-2 z_{0}\right)+(a+b+1) z_{0}-c, \quad Q_{n}=z_{0}\left(1-z_{0}\right)(n+s-2),
$$

with starting values

$$
d_{-1}\left(s, z_{0}\right)=0, \quad d_{0}\left(s, z_{0}\right)=1 .
$$

For the case that $b-a$ is an integer, a limiting process is needed (as in the next section). Details of the case $a=b$ are given in (Bühring 1987), where different representations of the coefficients in the series of (2.26) are also given.

When we take $z_{0}=\frac{1}{2}$ then the series in (2.26) converge outside the circle $\left|z-\frac{1}{2}\right|=\frac{1}{2}$, and both points $z=e^{ \pm \pi i / 3}$ discussed earlier are inside the domain of convergence.

\section{Removable singularities}

As mentioned earlier, there are several connection formulas that enable computation of the ${ }_{2} F_{1}$ - outside the unit disk. One example is

$$
\begin{aligned}
& { }_{2} F_{1}\left(\begin{array}{c}
a, b \\
c
\end{array} ; z\right)=\frac{\Gamma(c) \Gamma(c-a-b)}{\Gamma(c-a) \Gamma(c-b)}{ }_{2} F_{1}\left(\begin{array}{c}
a, b \\
a+b-c+1
\end{array} ; 1-z\right) \\
& \quad+\frac{\Gamma(c) \Gamma(a+b-c)}{\Gamma(a) \Gamma(b)}(1-z)^{c-a-b}{ }_{2} F_{1}\left(\begin{array}{c}
c-a, c-b \\
c-a-b+1
\end{array} ; 1-z\right) .
\end{aligned}
$$

For some combinations of the parameters $a, b$ and $c$ the relation in (2.30) cannot be used straightforwardly. For example, when $c=a+b$, the gamma functions $\Gamma(c-a-b)$ and $\Gamma(a+b-c)$ are not defined, and the two Gauss functions become the same. From an analytical point of view the limiting form of (2.30) exists when $c \rightarrow a+b$, but from a numerical point of view instabilities arise when the relation in (2.30) is used in that case. 
To see what happens in the limit $c \rightarrow a+b$, we write $c=a+b+\varepsilon$. We expand the Gauss functions in (2.30) in powers of $1-z$ and obtain for the $n$th term

$$
\frac{\Gamma(a+b+\varepsilon) \Gamma(1+\varepsilon) \Gamma(1-\varepsilon)(1-z)^{n}}{\Gamma(a) \Gamma(b) \Gamma(a+\varepsilon) \Gamma(b+\varepsilon) \Gamma(1-\varepsilon+n) \Gamma(1+\varepsilon+n) n !} f(\varepsilon),
$$

where

$$
\begin{aligned}
f(\varepsilon)= & \frac{1}{\varepsilon}(\Gamma(a+n) \Gamma(b+n) \Gamma(1+\varepsilon+n) \\
& \left.-(1-z)^{\varepsilon} \Gamma(a+\varepsilon+n) \Gamma(b+\varepsilon+n) \Gamma(1-\varepsilon+n)\right) .
\end{aligned}
$$

Taking the limit $\varepsilon \rightarrow 0$ in $f(\varepsilon)$, we find

$$
\begin{aligned}
& { }_{2} F_{1}\left(\begin{array}{c}
a, b \\
a+b
\end{array} ; z\right)=\frac{\Gamma(a+b)}{\Gamma(a) \Gamma(b)} \times \\
& \sum_{n=0}^{\infty} \frac{(a)_{n}(b)_{n}}{n ! n !}(2 \psi(n+1)-\psi(a+n)-\psi(b+n)-\ln (1-z))(1-z)^{n},
\end{aligned}
$$

where $\psi(z)$ is the logarithmic derivative of the gamma function:

$$
\psi(z)=\frac{\Gamma^{\prime}(z)}{\Gamma(z)} .
$$

This expansion holds for $|z-1|<1$ with $|\operatorname{ph}(1-z)|<\pi$. Thus there is, as usual, a branch cut from $z=1$ to $z=+\infty$, and $z$ is not on this cut. The logarithm $\ln (1-z)$ assumes its principal branch, which is real for $z<1$.

Gauss hypergeometric functions: Concluding remarks

We conclude with the following observations.

- The power series (2.19) of the Gauss hypergeometric function is a very important representation for numerical evaluations of this function.

- Non-trivial problems may arise, even for real values of the parameters and argument.

- With the transformation formulas that write the function in terms of functions with arguments shown in (2.24), we cannot reach all points in the complex $z$-plane.

- These formulas may cause numerical difficulties for certain combinations of the parameters $a, b$ and $c$ because of removable singularities in the formulas.

- In (Forrey 1997) many details are discussed for the numerical use of the transformation formulas, and details of a Fortran program are given.

- Other instabilities will arise in the power series evaluation when $a$ and/or $b$ and/or $c$ assume large (complex) values. 
- Some of the problems with these large parameters can be resolved by using recurrence relations for these functions. See Section 4 for more details, in particular Section 4.3.

\subsection{Chebyshev expansions of special functions}

A function $f$ that is continuous and of finite variation in $[-1,1]$ can be expanded in terms of Chebyshev polynomials, and the expansion given by

$$
f(x)=\frac{1}{2} c_{0}+\sum_{k=1}^{\infty} c_{k} T_{k}(x), \quad-1 \leq x \leq 1 .
$$

By using the orthogonality of $T_{n}(x)$, the coefficients of the expansion can be given as integrals in the form

$$
\begin{aligned}
c_{k} & =\frac{2}{\pi} \int_{-1}^{1} f(x) T_{k}(x)\left(1-x^{2}\right)^{-1 / 2} \mathrm{~d} x \\
& =\frac{2}{\pi} \int_{0}^{\pi} f(\cos \theta) \cos k \theta \mathrm{d} \theta \approx \frac{2}{n} \sum_{j=0}^{n} \prime \prime f\left(\cos \frac{\pi j}{n}\right) \cos \frac{\pi k j}{n},
\end{aligned}
$$

where the approximation is for sufficiently large $n$. The double prime over the summation indicates that the first and last terms are to be multiplied by $\frac{1}{2}$. Chebyshev coefficients are thus Fourier cosine transform coefficients of the function evaluated at non-uniformly spaced points.

As Clenshaw (Clenshaw 1957) explained, the coefficients in a Chebyshev expansion can also be obtained from recurrence relations when the function satisfies a linear differential equation with polynomial coefficients; see also (Gil, Segura and Temme 2007). All special functions of hypergeometric type satisfy such a differential equation. However, for many special functions we can obtain expansions in which the coefficients can be expressed in terms of known special functions. As an example, we have (see (Luke 1969a, p. 37))

$$
\begin{aligned}
& J_{0}(a x)=\sum_{n=0}^{\infty} \varepsilon_{n}(-1)^{n} J_{n}^{2}(a / 2) T_{2 n}(x), \\
& J_{1}(a x)=2 \sum_{n=0}^{\infty}(-1)^{n} J_{n}(a / 2) J_{n+1}(a / 2) T_{2 n+1}(x),
\end{aligned}
$$

where $-1 \leq x \leq 1$ and $\varepsilon_{0}=1, \varepsilon_{n}=2$ if $n>0$. The parameter $a$ can be any complex number. Similar expansions are available for $J$-Bessel functions of any complex order, in which the coefficients are ${ }_{1} F_{2}$-hypergeometric functions, and explicit recurrence relations are available for computing the coefficients. For integer orders, the coefficients are a product of two $J$-Bessel functions. Again, see (Luke 1969a). 
Remark 2.3. The complexity of computing the coefficients of the expansions in (2.37) seems to be greater than the computation of the function that has been expanded. In some sense this is true, but as we will see in the next section, the coefficients in (2.37), and those of many other examples for special functions, satisfy linear recurrence relations, and the coefficients satisfying such relations can usually be computed very efficiently by the socalled backward recursion algorithm. Details for the second-order recurrence relation will be given in the next section.

Another example is the expansion for the error function:

$$
e^{a^{2} x^{2}} \operatorname{erf}(a x)=\sqrt{\pi} e^{\frac{1}{2} a^{2}} \sum_{n=0}^{\infty} I_{n+\frac{1}{2}}\left(\frac{1}{2} a^{2}\right) T_{2 n+1}(x), \quad-1 \leq x \leq 1,
$$

in which the modified Bessel function is used. Again, $a$ can be any complex number.

The expansions in (2.37) and (2.38) can be viewed as expansions near the origin. Other expansions are available that can be viewed as expansions at infinity, and these may be considered as alternatives for asymptotic expansions of special functions. For example, for the confluent hypergeometric $U$-function defined in (2.13) we have the convergent expansion in terms of shifted Chebyshev polynomials $T_{n}^{*}(x)=T_{n}(2 x-1)$ :

$$
(\omega z)^{a} U(a ; c ; \omega z)=\sum_{n=0}^{\infty} C_{n}(z) T_{n}^{*}(1 / \omega), \quad z \neq 0, \quad|\operatorname{ph} z|<\frac{3}{2} \pi, \quad 1 \leq \omega \leq \infty .
$$

Furthermore, $a, 1+a-c \neq 0,-1,-2, \ldots$. When equalities hold for these values of $a$ and $c$ the Kummer function reduces to a Laguerre polynomial. This follows from

$$
U(a ; c ; z)=z^{1-c} U(1+a-c ; 2-c ; z)
$$

and

$$
U(-n ; \alpha+1 ; z)=(-1)^{n} n ! L_{n}^{\alpha}(z), \quad n=0,1,2, \ldots
$$

The expansion (2.39) is given in (Luke 1969a, p. 25). The coefficients can be represented in terms of generalized hypergeometric functions, in fact, Meijer $G$-functions, and they can be computed from the recurrence relation

$$
\frac{2 C_{n}(z)}{\varepsilon_{n}}=2(n+1) A_{1} C_{n+1}(z)+A_{2} C_{n+2}(z)+A_{3} C_{n+3}(z),
$$


where $b=a+1-c, \varepsilon_{0}=\frac{1}{2}, \varepsilon_{n}=1(n \geq 1)$, and

$$
\begin{aligned}
& A_{1}=1-\frac{(2 n+3)(n+a+1)(n+b+1)}{2(n+2)(n+a)(n+b)}-\frac{2 z}{(n+a)(n+b)}, \\
& A_{2}=1-\frac{2(n+1)(2 n+3-z)}{(n+a)(n+b)} \\
& A_{3}=-\frac{(n+1)(n+3-a)(n+3-b)}{(n+2)(n+a)(n+b)} .
\end{aligned}
$$

Again, the coefficients satisfying this third-order recurrence relation can be computed by a backward recursion algorithm. For applying the backward recursion it is important to know that we have the normalization relation

$$
\sum_{n=0}^{\infty}(-1)^{n} C_{n}(z)=1, \quad|\operatorname{ph} z|<\frac{3}{2} \pi .
$$

This follows from

$$
\lim _{\omega \rightarrow \infty}(\omega z)^{a} U(a ; c ; \omega z)=1, \quad \text { and } \quad T_{n}^{*}(0)=(-1)^{n},
$$

and from the asymptotic expansion given in (2.18). The standard backward recursion scheme for computing the coefficients $C_{n}(z)$ only works for $|\mathrm{ph} z|<$ $\pi$. For $\mathrm{ph} z= \pm \pi$ a modification seems to be possible; see (Luke 1969a, p. 26).

Although the expansion in (2.39) converges for all $z \neq 0$ in the indicated sector, it is better to avoid small values of the argument of the $U$-function. Luke gives an estimate of the coefficients $C_{n}(z)$ of which the dominant factor that determines the speed of convergence is given by

$$
C_{n}(z)=\mathcal{O}\left(n^{2(2 a-c-1) / 3} e^{-3 n^{\frac{2}{3}} z^{\frac{1}{3}}}\right), \quad n \rightarrow \infty,
$$

and we see that large values of $\operatorname{Re} z^{\frac{1}{3}}$ improve the convergence.

The expansion in (2.39) can be used for all special cases of the Kummer $U$-function, that is, for Bessel functions (Hankel functions and $K$-modified Bessel functions), for the incomplete gamma function $\Gamma(a, z)$, with as special cases the complementary error function and exponential integrals.

Example 2.4. (Airy function) For the Airy function $\operatorname{Ai}(x)$ we have the relations

$$
\begin{aligned}
& \xi^{\frac{1}{6}} U\left(\frac{1}{6} ; \frac{1}{3} ; \xi\right)=2 \sqrt{\pi} x^{\frac{1}{4}} e^{\frac{1}{2} \xi} \mathrm{Ai}(x), \\
& \xi^{-\frac{1}{6}} U\left(-\frac{1}{6} ;-\frac{1}{3} ; \xi\right)=-2 \sqrt{\pi} x^{-\frac{1}{4}} e^{\frac{1}{2} \xi} \operatorname{Ai}^{\prime}(x),
\end{aligned}
$$

where $\xi=\frac{4}{3} x^{\frac{3}{2}}$. We take $\omega=(x / 7)^{3 / 2}$ and $z=\frac{4}{3} 7^{3 / 2}=24.69 \ldots$

To generate the coefficients with this value of $z$ we determine the smallest 
Table 2.1. Coefficients of the Chebyshev expansion (2.51)

\begin{tabular}{|c|c|c|}
\hline$n$ & $C_{n}(z)$ & $D_{n}(z)$ \\
\hline 0 & 0.997273395501425 & 1.003835579657251 \\
\hline 1 & -0.002698958707030 & 0.003802737406686 \\
\hline 2 & 0.000027127484648 & -0.000032259878104 \\
\hline 3 & -0.000000504354523 & 0.000000567125559 \\
\hline 4 & 0.000000013468935 & -0.000000014727362 \\
\hline 5 & -0.000000000463150 & 0.000000000497977 \\
\hline 6 & 0.000000000019298 & -0.000000000020517 \\
\hline 7 & -0.000000000000938 & 0.000000000000989 \\
\hline 8 & 0.000000000000052 & -0.000000000000054 \\
\hline 9 & -0.000000000000003 & 0.000000000000003 \\
\hline
\end{tabular}

value of $n$ for which the exponential factor in (2.46) is smaller than $10^{-15}$. This gives $n=8$. Next we generate, for both $U$ - functions in (2.47), the coefficients $C_{n}(z)$ by using (2.42) in the backward direction. Details of this method for second-order recurrence relations are given in Section 4.

We take starting values

$$
\widetilde{C}_{19}(z)=1, \quad \widetilde{C}_{20}(z)=0, \quad \widetilde{C}_{21}(z)=0 .
$$

We also compute for normalization

$$
S=\sum_{n=0}^{18}(-1)^{n} \widetilde{C}_{n}(z)=-0.902363242772764_{10}{ }^{25},
$$

where the numerical value is for the Ai-case. Finally we compute

$$
C_{n}(z)=\widetilde{C}_{n}(z) / S, \quad n=0,1,2, \ldots, 10 .
$$

Using this scheme for the expansions (2.39) of both $U$-functions in (2.47), we obtain the coefficients $C_{n}(z)$ and $D_{n}(z)$ of the expansions

$$
\begin{aligned}
& 2 \sqrt{\pi} x^{\frac{1}{4}} e^{\frac{2}{3} x^{3 / 2}} \operatorname{Ai}(x) \approx \sum_{n=0}^{9} C_{n} T_{n}^{*}\left((7 / x)^{3 / 2}\right), \\
& -2 \sqrt{\pi} x^{\frac{1}{4}} e^{\frac{2}{3} x^{3 / 2}} \operatorname{Ai}^{\prime}(x) \approx \sum_{n=0}^{9} D_{n} T_{n}^{*}\left((7 / x)^{3 / 2}\right),
\end{aligned}
$$

for $x \geq 7$, for which numerical values are given in Table 2.1. 


\section{Numerical quadrature}

The many special functions that we meet in applications can usually be defined by integral representations. For straightforward use these integrals may not be the optimal choice for numerical quadrature. In many cases we like to transform the integral into a different one with a better condition for numerical computations. The condition can be improved by considering two important points.

- The original integral may behave badly because of strong oscillations, and the new representation may be free of strong oscillations.

- The integral may be very large or very small (because of large parameters) and with the transformation a dominant factor has been placed in front of the new integral.

For an example of the first point, consider the ordinary Bessel function of the first kind with integer order, which can be defined as (Abramowitz and Stegun 1964, Equation 9.1.21)

$$
J_{n}(z)=\frac{1}{2 \pi} \int_{-\pi}^{\pi} \cos (z \sin \theta-n \theta) \mathrm{d} \theta, \quad n \in \mathbb{Z}, \quad z \in \mathbb{C} .
$$

This function has for fixed $z$ the asymptotic behaviour (Abramowitz and Stegun 1964, 9.3.1)

$$
J_{n}(z) \sim \frac{1}{\sqrt{2 \pi n}}\left(\frac{e z}{2 n}\right)^{n}, \quad n \rightarrow \infty,
$$

and, for obtaining a high relative precision, the integral in (3.1) becomes useless when $n$ is large. There is no simple transformation possible for this integral to extract the dominant asymptotic factor, and a different method should be used, based on contour integrals in the complex plane, to obtain an integral with a better condition.

In the case of the gamma function

$$
\Gamma(z)=\int_{0}^{\infty} t^{z-1} e^{-t} \mathrm{~d} t, \quad \operatorname{Re} z>0,
$$

a simple transformation $t=z s$ (first assuming that $z>0$ ) gives

$$
\Gamma(z)=z^{z} e^{-z} \int_{0}^{\infty} e^{-z \phi(s)} \frac{\mathrm{d} s}{s}, \quad \phi(s)=s-\log s-1,
$$

and the integral is now $\mathcal{O}(1 / \sqrt{z})$ as $z \rightarrow \infty$.

Another important decision when using numerical quadrature for the computation of special functions is the choice of the quadrature rule or method. Because the integrals for special functions are always given in terms of integrands that are analytic functions (except possibly at the endpoints, as in 
the case of (3.3) and (3.4) at the origin) the conditions for the remainders are usually quite favourable.

In addition, we like to use an adaptive rule that can easily be applied with any number of nodes and weights, without calculating these quantities a priori. Of all known quadrature rules the trapezoidal rule is one of the simplest to apply and it has the property that no nodes and weights have to be computed. Moreover, when halving the stepsize for the nodes, earlier function values can be used in the finer rule.

\subsection{The finite interval}

We recall two forms of the $n$-point extended trapezoidal rule (Davis and Rabinowitz 1984). Let $n=1,2,3, \ldots$ and

$$
h=\frac{b-a}{n} .
$$

Then the standard rule reads

$$
\int_{a}^{b} f(t) \mathrm{d} t=\frac{1}{2} h[f(a)+f(b)]+h \sum_{j=1}^{n-1} f(j h)+R_{n},
$$

and the modified or shifted rule is given by

$$
\int_{a}^{b} f(t) \mathrm{d} t=h \sum_{j=0}^{n-1} f(a+d+j h)+R_{n}^{d}, \quad 0<d<h .
$$

In both cases the standard theory gives that the error terms have the form

$$
R_{n}, R_{n}^{d}=-\frac{n h^{3}}{12} f^{\prime \prime}(\xi),
$$

for some point $\xi \in(a, b)$, and for functions with continuous second derivative on $[a, b]$.

Because of this form of the remainder $R_{n}$ the standard trapezoidal rule is not a high precision rule, but under certain conditions the rule is extremely accurate. We will describe these conditions and will give several examples to show the efficiency of the rule.

We apply the trapezoidal rule to functions belonging to two classes, one for finite and one for infinite intervals. For finite integrals the class is defined as follows.

Definition 3.1. Let $C_{p}^{\infty}([a, b])$ denote the class of $C^{\infty}$-functions

$$
f:[a, b] \mapsto \mathbb{C}, \quad-\infty<a<b<\infty,
$$

with equal derivatives of all orders at $a$ and $b$ :

$$
f^{(n)}(a)=f^{(n)}(b), \quad n=0,1,2, \ldots .
$$


Table 3.2. Values of the remainders in the trapezoidal rule for the Bessel function $J_{0}(x)$ by using (3.11) and (3.12). The rightmost column gives the upper bound $\left|R_{n}\right|$ based on (3.13).

\begin{tabular}{||c|c|c|c}
\hline$n$ & $R_{n}$ & $R_{n}^{h / 2}$ & bound of $\left|R_{n}\right|$ \\
\hline 4 & $-0.12_{10}(-000)$ & $0.12_{0}(-000)$ & $0.92_{10}(-000)$ \\
8 & $-0.48_{10}(-006)$ & $0.48_{0}(-006)$ & $0.27_{10}(-005)$ \\
16 & $-0.11_{10}(-021)$ & $0.11_{10}(-021)$ & $0.59_{10}(-021)$ \\
32 & $-0.13_{10}(-062)$ & $0.13_{10}(-062)$ & $0.56_{10}(-062)$ \\
64 & $-0.13_{10}(-163)$ & $0.13_{10}(-163)$ & $0.54_{10}(-163)$ \\
128 & $-0.533_{10}(-404)$ & $0.53_{10}(-404)$ & $0.21_{10}(-403)$ \\
\hline
\end{tabular}

Remark 3.2. The functions of $C_{p}^{\infty}([a, b])$ met in this paper can often be continued analytically so as to be single-valued and regular in a region $D \subset \mathbb{C}$ containing $(a, b)$. They can be continued outside the interval $[a, b]$ to become $C^{\infty}$-periodic functions on $\mathbb{R}$ with period $b-a$.

As we will show later, the trapezoidal rule on $[a, b]$ is extremely efficient for functions belonging to $C_{p}^{\infty}([a, b])$. The integrand $\cos (z \sin \theta-n \theta)$ of $(3.1)$ belongs to $C_{p}^{\infty}([-\pi, \pi])$. For moderate values of $n$ and $z$ the trapezoidal is indeed very efficient, as follows from the following example.

Example 3.3. (The Bessel function $\boldsymbol{J}_{\mathbf{0}}(\boldsymbol{x})$ ) We take (3.1) over the interval $[0, \pi]$. Using (3.6) and (3.7) with $d=\frac{1}{2} h$, we obtain

$$
\begin{gathered}
\pi J_{0}(x)=\int_{0}^{\pi} \cos (x \sin t) \mathrm{d} t=h+h \sum_{j=1}^{n-1} \cos [x \sin (j h)]+R_{n}, \\
\pi J_{0}(x)=\int_{0}^{\pi} \cos (x \sin t) \mathrm{d} t=h \sum_{j=0}^{n-1} \cos \left[x \sin \left(\frac{1}{2} h+j h\right)\right]+R_{n}^{h / 2},
\end{gathered}
$$

where $h=\pi / n$. We take $x=5$ and have the results as shown in Table 3.2.

We observe that the error terms $R_{n}$ and $R_{n}^{h / 2}$ are much smaller than the upper bound that can be obtained from (3.8). Also, comparison of the 
two remainders (and considering more digits) shows that the differences in accuracy is negligible. In addition, $R_{n}$ and $R_{n}^{h / 2}$ have opposite signs. This phenomenon often occurs and will be explained later.

For more details we refer to (Davis and Rabinowitz 1984). (Luke 1969b, p. 218) considered this Bessel function integral in detail, and from this reference and (Krumhaar 1965) we derive an upper bound for $R_{n}$,

$$
\left|R_{n}\right| \leq 2 e^{x / 2} \frac{(x / 2)^{2 n}}{(2 n) !}
$$

which is quite realistic for the value of $x$ we have chosen. For a different representation of the remainder see Example 3.8.

\section{Remainder estimate with higher derivatives}

Other representations of the remainders which are completely different from those in (3.8) are related to Euler's summation formula; see (Temme 1996, Section 1.1.3).

First we introduce a function $\widetilde{B}_{m}(x)$ that is related with the generalized Bernoulli polynomial $B_{m}(x)$, which is defined by the generating function relation

$$
\frac{z e^{x z}}{e^{z}-1}=\sum_{n=0}^{\infty} \frac{B_{m}(x)}{m !} z^{m}, \quad|z|<2 \pi .
$$

Explicit representations of $B_{m}(x)$ are (see Abramowitz and Stegun (1964, Equations 23.1.17-23.1.18))

$$
B_{2 m}(x)=\frac{2(-1)^{m+1}(2 m) !}{(2 \pi)^{2 m}} \sum_{k=1}^{\infty} \frac{\cos (2 \pi k x)}{k^{2 m}}, \quad 0 \leq x \leq 1, \quad m \geq 1
$$

and

$$
B_{2 m+1}(x)=\frac{2(-1)^{m+1}(2 m+1) !}{(2 \pi)^{2 m+1}} \sum_{k=1}^{\infty} \frac{\sin (2 \pi k x)}{k^{2 m+1}}, \quad 0 \leq x \leq 1, \quad m \geq 0 .
$$

The expansion for $B_{1}(x)$ holds for $0<x<1$.

The functions $\widetilde{B}_{m}(x)$ are defined as the periodic continuations of $B_{m}(x)$ outside the basic interval $[0,1]$. That is,

$$
\widetilde{B}_{m}(x)= \begin{cases}B_{m}(x) & \text { if } 0 \leq x \leq 1 \\ \widetilde{B}_{m}(x-1) & \text { if } x \in \mathbb{R}\end{cases}
$$

In other words, $\widetilde{B}_{m}(x), m \geq 2$, can also be defined by (3.15) and (3.16), with $x \in \mathbb{R}$. 
Theorem 3.4. Suppose that $f$ is differentiable in $[a, b]$ up to and including order $2 m+1$. Then we have

$$
R_{n}=-\sum_{k=1}^{m} \frac{h^{2 k} B_{2 k}}{(2 k) !}\left[f^{(2 k-1)}(b)-f^{(2 k-1)}(a)\right]+R_{n, m},
$$

where

$$
R_{n, m}=-\frac{h^{2 m+1}}{(2 m+1) !} \int_{a}^{b} f^{(2 m+1)}(t) \widetilde{B}_{2 m+1}\left(\frac{s-a}{h}\right) \mathrm{d} s
$$

Proof. See (Temme 1996, Exercise 1.7, p. 23).

This result corresponds to Euler's summation formula on finite intervals, in which a finite sum is expressed in terms of an integral plus terms and remainder of the form just given.

Corollary 3.5. (Euler's summation formula) Suppose that $f$ is differentiable in $[0, n]$ up to and including order $2 m+1, n \geq 1$. Then

$$
\begin{aligned}
\sum_{j=0}^{n} f(j)= & \int_{0}^{n} f(x) \mathrm{d} x+\frac{1}{2}[f(0)+f(n)]+ \\
& \sum_{j=1}^{m} \frac{B_{2 j}}{(2 j) !}\left[f^{(2 j-1)}(n)-f^{(2 j-1)}(0)\right]+R_{n, m},
\end{aligned}
$$

where

$$
R_{n, m}=\frac{1}{(2 m+1) !} \int_{0}^{n} f^{(2 m+1)}(x) \widetilde{B}_{2 m+1}(x) \mathrm{d} x .
$$

For the modified rule (3.7) we have the following result

Theorem 3.6. Suppose that $f$ is differentiable in $[a, b]$ up to and including order $2 m+1$. Let $d=\frac{1}{2} h$. Then

$$
R_{n}^{h / 2}=-\sum_{k=1}^{m} \frac{h^{2 k} B_{2 k}\left(\frac{1}{2}\right)}{(2 k) !}\left[f^{(2 k-1)}(b)-f^{(2 k-1)}(a)\right]+R_{n, m}^{h / 2},
$$

where

$$
R_{n, m}^{h / 2}=-\frac{h^{2 m+1}}{(2 m+1) !} \int_{a}^{b} f^{(2 m+1)}(t) \widetilde{B}_{2 m+1}\left(\frac{s-a}{h}+\frac{1}{2}\right) \mathrm{d} s .
$$

Proof. The proof is as for Theorem 3.4 and also follows from (Luke 1969b, pp. 218-219) (with modifications and a few corrections).

Now, for $f \in C_{p}^{\infty}[a, b]$ (see Definition 3.1), we see that all terms in the 
sums of (3.18) and (3.22) vanish, and from (3.16) we infer

$$
\left|R_{n, m}\right|,\left|R_{n, m}^{h / 2}\right| \leq 2\left(\frac{h}{2 \pi}\right)^{2 m+1} \int_{a}^{b}\left|f^{(2 m+1)}(t)\right| \mathrm{d} t, \quad m=1,2,3, \ldots .
$$

This shows that for $f \in C_{p}^{\infty}[a, b]$ the trapezoidal rule has order $h^{2 m+1}$ for any $m \geq 1$. Because (3.16) implies that

$$
\widetilde{B}_{2 m+1}\left(x+\frac{1}{2}\right)=\frac{2(-1)^{m+1}(2 m+1) !}{(2 \pi)^{2 m+1}} \sum_{k=1}^{\infty}(-1)^{k} \frac{\sin (2 \pi k x)}{k^{2 m+1}}, \quad x \in \mathbb{R},
$$

we have also explained why the remainders $R_{n, m}$ and $R_{n, m}^{h / 2}$ are almost equal, and may have opposite signs.

Adding the two remainders we have

$$
\begin{aligned}
& R_{n, m}+R_{n, m}^{h / 2}= \\
& \quad-\frac{h^{2 m+1}}{(2 m+1) !} \int_{a}^{b} f^{(2 m+1)}(t)\left[\widetilde{B}_{2 m+1}\left(\frac{s-a}{h}\right)+\widetilde{B}_{2 m+1}\left(\frac{s-a}{h}+\frac{1}{2}\right)\right] \mathrm{d} s,
\end{aligned}
$$

and using (3.16) and (3.25) we see that

$$
\widetilde{B}_{2 m+1}(x)+\widetilde{B}_{2 m+1}\left(x+\frac{1}{2}\right)=\frac{1}{2^{2 m}} \frac{2(-1)^{m+1}(2 m+1) !}{(2 \pi)^{2 m+1}} \sum_{k=1}^{\infty} \frac{\sin (4 \pi k x)}{k^{2 m+1}} .
$$

It is obvious that when we add the standard trapezoidal rule (3.6) and the modified rule (3.7) with $d=\frac{1}{2} h$, we obtain the standard rule with $n$ replaced by $2 n$ (and with $h$ replaced by $h / 2$ ). From (3.26) and (3.27) we see that the sum of the remainders of both rules follows the same pattern. From Table 3.2 we see that doubling the number of terms initially triples the number of correct decimal digits.

Representation of the remainder based on Fourier series

In the next theorem we express the remainder of the trapezoidal rule in terms of the Fourier series of $f$.

Theorem 3.7. Assume that $f$ is continuous on $[a, b]$ with an absolutely and uniformly convergent Fourier series

$$
f(x)=\sum_{k=-\infty}^{\infty} a_{k} e^{\mathrm{i} \omega k x}, \quad a_{k}=\frac{\omega}{2 \pi} \int_{a}^{b} f(x) e^{-\mathrm{i} \omega k x} \mathrm{~d} x,
$$

where $\omega=2 \pi /(b-a)$, then $R_{n}$ of (3.6) is given by

$$
R_{n}=-(b-a) \sum_{\substack{m=-\infty \\ m \neq 0}}^{\infty} e^{\mathrm{i} \omega a m n} a_{m n}
$$


For $R_{n}^{d}$ of (3.7) we have

$$
R_{n}^{d}=-(b-a) \sum_{\substack{m=-\infty \\ m \neq 0}}^{\infty} e^{\mathrm{i} \omega m n(a+d-h)} a_{m n} .
$$

Proof. First consider $R_{n}$ for a single Fourier term. That is, we consider (3.6) with $f_{k}(x)=e^{\mathrm{i} \omega k x}$. The integral vanishes, and consequently

$$
R_{h}=\frac{1}{2} h\left[f_{k}(b)-f_{k}(a)\right]-h e^{\mathrm{i} \omega a k} \sum_{j=1}^{n} e^{\mathrm{i} \omega h j k},
$$

where $f_{k}(a)=f_{k}(b)$. From the well-known result

$$
\sum_{j=1}^{n} e^{\mathrm{i} j \theta}=e^{\frac{1}{2} \mathrm{i}(n+1) \theta} \frac{\sin (n \theta / 2}{\sin (\theta / 2)},
$$

we easily find, with $\theta=\omega h k=2 \pi k / n$ for $m= \pm 1, \pm 2, \ldots$,

$$
R_{n}= \begin{cases}0 & \text { if } k \neq m n, \\ -(b-a) e^{\mathrm{i} \omega a m n} & \text { if } k=m n,\end{cases}
$$

Using this result for $f_{k}(x)=e^{\mathrm{i} \omega k x}$ for all terms in the Fourier series of (3.28), we find the claim in (3.29). The result (3.30) for $R_{n}^{d}$ follows easily.

When $d=\frac{1}{2} h$ in (3.30), we have

$$
R_{n}^{d}=-(b-a) \sum_{\substack{m=-\infty \\ m \neq 0}}^{\infty}(-1)^{m} e^{\mathrm{i} \omega m n a} a_{m n} .
$$

and when the Fourier expansion converges fast and we consider the first term approximations $m= \pm 1$ of (3.29) and (3.30), we have

$$
R_{n} \sim-(b-a)\left[e^{\mathrm{i} \omega a n} a_{n}+e^{-\mathrm{i} \omega a n} a_{-n}\right], \quad R_{n}^{h / 2} \sim-R_{n} .
$$

Example 3.8. ([The Bessel function $\boldsymbol{J}_{\mathbf{0}}(\boldsymbol{x})$ ) Consider the Fourier series (Abramowitz and Stegun 1964, Equation 9.1.42)

$$
\cos (z \sin x)=\sum_{k=-\infty}^{\infty} J_{2 k}(z) \cos (2 k x) .
$$

We have $a=0, b=\pi, \omega=2, a_{k}=J_{2 k}(z)$, and $J_{-k}(z)=(-1)^{k} J_{k}(z)$. The integral of the left-hand side equals $\pi J_{0}(z)$ (see (3.12)). For the remainder we have

$$
R_{n}=-2 \pi \sum_{m=1}^{\infty} J_{2 m n}(z) \text {. }
$$


This gives an exact form of the remainder $R_{n}$ of (3.11).

Contour integrals for the remainders

Representations of the remainders in (3.6) and (3.7) can be given in the form of contour integrals in the complex plane in which $f$ but no derivatives of $f$ are used. For details we refer to (Takahasi and Mori 1970, Mori 1974).

\subsection{The infinite interval}

For the trapezoidal rule on $\mathbb{R}$, we consider for a certain class of $f$,

$$
\int_{-\infty}^{\infty} f(t) \mathrm{d} t=\sum_{j=-\infty}^{\infty} f(d+j h)+R_{d}(h), \quad 0 \leq d<h .
$$

This class is described as follows.

Definition 3.9. Let

$$
G_{a}=\{z=x+\mathrm{i} y|x \in \mathbb{R},| y \mid<a\}
$$

be the strip in the complex domain of width $2 a>0$. Let $H_{a}$ denote the class of bounded holomorphic functions $f: G_{a} \mapsto \mathbb{C}$ such that $\int_{-\infty}^{\infty} f(x+\mathrm{i} y) \mathrm{d} x$ converges, uniformly with respect to $y \in[-a, a]$, with $\lim _{x \rightarrow \pm \infty} f(x+\mathrm{i} y)=$ 0 , uniformly with respect to $y \in[-a, a]$, and

$$
M_{ \pm a}(f)=\int_{-\infty}^{\infty}|f(x \pm \mathrm{i} a)| \mathrm{d} x=\lim _{b \uparrow a} \int_{-\infty}^{\infty}|f(x \pm \mathrm{i} b)| \mathrm{d} x<\infty .
$$

For the remainder $R_{d}(h)$ in (3.38) we have the following result.

Theorem 3.10. Let $f \in H_{a}$ for some $a>0$. Let $h>0$ and $0 \leq d<h$. Then

$$
R_{d}(h)=\int_{-\infty}^{\infty} \frac{f(x+\mathrm{i} y)}{1-e^{-2 \pi \mathrm{i}(x+\mathrm{i} y-d) / h}} \mathrm{~d} x+\int_{-\infty}^{\infty} \frac{f(x-\mathrm{i} y)}{1-e^{2 \pi \mathrm{i}(x-\mathrm{i} y-d) / h}} \mathrm{~d} x,
$$

for any $y \in(0, a)$.

Proof. The known proof is based on residue calculus for evaluating integrals. Observe that

$$
\frac{1}{2 \pi \mathrm{i}} \int_{\partial G_{y}} f(z) \cot (\pi(z-d) / h) \mathrm{d} z=h \sum_{j=-\infty}^{\infty} f(d+j h),
$$

where $\partial G_{y}$ is the boundary of $G_{y}$ (see (3.39)), and the integration in (3.42) is in the positive direction. Furthermore,

$$
\int_{-\infty}^{\infty} f(x) \mathrm{d} x+\int_{\infty}^{-\infty} f(x+\mathrm{i} y) \mathrm{d} x=0
$$


and

$$
\int_{-\infty}^{\infty} f(x) \mathrm{d} x+\int_{\infty}^{-\infty} f(x-\mathrm{i} y) \mathrm{d} x=0 .
$$

Combining these results we arrive at (3.41).

Corollary 3.11. Let $f \in H_{a}$ for some $a>0$ and let $f$ be even (which is not a restriction for integrals over $\mathbb{R}$ ). Then $R_{d}(h)$ in (3.38) can be bounded in the following way:

$$
\left|R_{d}(h)\right| \leq \frac{e^{-\pi a / h}}{\sinh (\pi a / h)} M_{a}(f),
$$

where $M_{a}(f)$ is given in (3.40).

From (3.45) it follows that the error in the trapezoidal rule for small $h$ is $\mathcal{O}(\exp (-2 \pi a / h))$. Also, large values of $a$ result in small errors, but the quantity $M_{a}(f)$ may influence the error if $a$ is large. Often, it is advisable to choose $h$ and $a$ such that the right-hand side of (3.45) is minimized.

For example, when $f$ has the form

$$
f(x)=e^{-\omega x^{2}} g(x), \quad \omega>0, \quad g \in H_{a},
$$

for some $a>0$ and $g$ an even function, (3.45) can be written as

$$
\left|R_{d}(h)\right| \leq \frac{e^{\omega a^{2}-\pi a / h}}{\sinh (\pi a / h)} M_{a}(g) .
$$

If $g$ allows, we may choose a value of $a$ that makes the exponential function very large. However, the function $-2 \pi a / h+\omega a^{2}$, considered as a function of $a$, is minimal for

$$
a=\frac{\pi}{\omega h} .
$$

When we can take this value (again, if $g$ allows), and we suppose that $M_{a}(g)=\mathcal{O}(1)$, we conclude that the error satisfies

$$
\left|R_{d}(h)\right| \leq C e^{-\pi^{2} /\left(\omega h^{2}\right)}, \text { for some } C>0 .
$$

Remark 3.12. In applications we may be interested in large values of $\omega$. If we apply the bound in (3.49) to make $\left|R_{d}(h)\right|<\varepsilon$, we see that $h$ should satisfy $h \sim \pi / \sqrt{\omega \log (1 / \varepsilon)}$, which may be quite small, when $\omega$ is large. On the other hand, we need to consider the rate of convergence of the series in (3.38) with this value of $h$ and $\omega$ for the function in (3.46). We see that $f(j h)=\exp \left(-\omega j^{2} h^{2}\right) g(j h)$ and that $\omega j^{2} h^{2}=\pi^{2} j^{2} / \log (1 / \varepsilon)$. So, the value of $j$ that should be taken to neglect terms in the infinite series is not dependent on $\omega$, when we take $h$ as mentioned earlier. It is of some value to know the stepsize $h$, but for efficiency the number of terms is the most important issue. Of course, we can also take a new variable of integration 
$x=t / \sqrt{\omega}$ when $f$ has the form of (3.46). In that case the number of terms in the series may again become independent of $\omega$, and the singularities of $g$ are moving off the real axis as $\omega$ increases.

Another representation of the remainder

Another representation for $R_{d}(h)$ can be obtained by using Poisson's summation formula, which we write in the general form

$$
h \sum_{j=-\infty}^{\infty} e^{\mathrm{i} j \alpha} f(d+j h)=\sum_{k=-\infty}^{\infty} F\left(\frac{2 \pi k+\alpha}{h}\right) e^{-\mathrm{i} d(2 \pi k+\alpha) / h},
$$

where $F$ is the Fourier transform of $f$ :

$$
F(y)=\int_{-\infty}^{\infty} f(x) e^{\mathrm{i} x y} \mathrm{~d} x .
$$

Details on the Poisson summation formula and condition of validity can be found in (Andrews, Askey and Roy 1999, Section D.4).

For $\alpha=0$ we obtain a representation for $R_{d}(h)$ of $(3.38)$

$$
R_{d}(h)=\sum_{k \neq 0} F\left(\frac{2 \pi k}{h}\right) e^{-2 \pi \mathrm{i} k d / h} .
$$

For even functions this becomes

$$
R_{d}(h)=2 \sum_{k=1}^{\infty} F\left(\frac{2 \pi k}{h}\right) \cos (2 \pi k d / h) .
$$

For functions $f \in H_{a}$ this result can also be obtained by expanding in (3.41) the integrands in geometric series with exponential functions.

Remark 3.13. Observe that, when the series converges fast, and the first term gives a good estimate of the error, the remainders $R_{0}(h)$ and $R_{h / 2}(h)$ are almost equal in modulus and have opposite sign.

\section{Including series with derivatives}

An interesting generalization of (3.38) is based on the idea of Hermite interpolation. The result is summarized in the following theorem.

Theorem 3.14. Let $f \in H_{a}$, for some $a>0$, be an even function. For any positive integer $p$, let numbers $a_{q, p}$ be determined by the identity

$$
c_{0, p}+c_{2, p} z^{2}+\cdots+c_{p, p} z^{p}=\prod_{q=1}^{\frac{1}{2} p}\left((1+(z / q))^{2}\right) .
$$

Then, for $h>0$,

$$
\int_{-\infty}^{\infty} f(x) \mathrm{d} x=T_{p, h}(f)+R_{p, h}(f)
$$


where

$$
T_{p, h}(f)=h \sum_{\substack{q=0 \\ q \text { even }}}^{p} c_{q, p}\left(\frac{h}{2 \pi}\right)^{q} \sum_{j=-\infty}^{\infty} f^{(q)}(j h),
$$

and $R_{p, h}(f)$ is bounded as follows:

$$
\left|R_{p, h}(f)\right| \leq \frac{e^{-\pi a / h}}{\sinh ^{p+1}(\pi a / h)} M_{a}(f) .
$$

Proof. For a proof we refer to (Kreß 1972).

We give the rules with derivatives for $p=0,2,4$ :

$$
\begin{aligned}
& T_{0, h}(f)=h \sum_{j=-\infty}^{\infty} f(j h), \\
& T_{2, h}(f)=T_{0, h}(f)+\frac{h^{3}}{4 \pi^{2}} \sum_{j=-\infty}^{\infty} f^{\prime \prime}(j h), \\
& T_{4, h}(f)=T_{0, h}(f)+\frac{5 h^{3}}{16 \pi^{2}} \sum_{j=-\infty}^{\infty} f^{\prime \prime}(j h)+\frac{h^{5}}{64 \pi^{4}} \sum_{j=-\infty}^{\infty} f^{\prime \prime \prime \prime}(j h) .
\end{aligned}
$$

The coefficients $c_{q, p}$, of which the first few appear in (3.58), can easily be obtained as follows. By differentiating (3.38) with respect to $d$ and by using (3.52) for $R_{d}(h)$, we obtain (by taking $d=0$ afterwards) for even functions $f$ :

$$
\begin{aligned}
\int_{-\infty}^{\infty} f(x) \mathrm{d} x & =h \sum_{j=-\infty}^{\infty} f(j h) \\
0 & =h \sum_{j=-\infty}^{\infty} f^{\prime \prime}(j h)+\frac{8 \pi^{2}}{h^{2}} \sum_{k=1}^{\infty} k^{2} F\left(\frac{2 \pi k}{h}\right), \\
0 & =h \sum_{j=-\infty}^{\infty} f^{\prime \prime \prime \prime}(j h)-\frac{32 \pi^{4}}{h^{4}} \sum_{k=1}^{\infty} k^{4} F\left(\frac{2 \pi k}{h}\right),
\end{aligned}
$$

and so on. By taking linear combinations of these equations we can eliminate $F(2 \pi / h), F(4 \pi / h), \ldots$, and the coefficients for these linear combinations are the numbers $c_{q, p}$. This procedure reminds us of the Romberg method, where terms in the error representation are eliminated by taking linear combinations of the trapezoidal rule with $h, h / 2, h / 4, \ldots$.

Example 3.15. ( $\left.\boldsymbol{f}(\boldsymbol{x})=e^{-\boldsymbol{x}^{2}}\right) \quad$ The remainder $R_{d}(h)$ follows from (3.52) 
and is given by

$$
R_{d}(h)=-2 \sqrt{\pi} \sum_{k=1}^{\infty} e^{-\pi^{2} k^{2} / h^{2}} \cos (2 \pi k d / h) .
$$

The first term can be compared with the estimate in (3.49) when $\omega=1$. For an accuracy of $10^{-10}, R_{d}(h)$ is negligible for $h$ smaller than (approximately) $\pi / \sqrt{10 \log (10)}=0.65 \cdots$. With this value we can estimate the number of terms in the infinite sum in (3.38). The terms are negligible for $j$ larger than $\sqrt{10 \log (10} / h=7.3 \cdots$.

To see the effect of including the series with the second derivative, we derive

$$
R_{p, h}(f)=2 \sqrt{\pi} \sum_{k=2}^{\infty}\left(k^{2}-1\right) e^{-\pi^{2} k^{2} / h^{2}} .
$$

To obtain the same accuracy as for (3.60) we can take $h=1.31$ and neglect terms in the infinite series for $j$ larger than 3.66. Hence, by using the second derivative the number of terms in the infinite series can be halved in this example.

Example 3.16. $\left(f(x)=1 /\left(1+x^{2}\right)\right)$ This case is only of theoretical interest, because the series in (3.38) does not converge fast enough. In any case, we have $f \in H_{a}, a \in(0,1)$, and we can evaluate

$$
R_{d}(h)=-2 \pi \sum_{k=1}^{\infty} e^{-2 \pi k / h} \cos (2 \pi k d / h)=-\frac{2 \pi}{e^{2 \pi / h}-1},
$$

where the last result holds when $d=0$. So, although the rule is useless, the error bound is perfect.

Slowly convergent integrals can become more rapidly convergent by using simple transformations. In the present case we use $x=\sinh t$ and obtain

$$
\int_{-\infty}^{\infty} \frac{\mathrm{d} x}{1+x^{2}}=\int_{-\infty}^{\infty} \frac{\mathrm{d} t}{\cosh t}
$$

The new integrand belongs to $H_{a}, a \in\left(0, \frac{1}{2} \pi\right)$, and

$$
R_{d}(h)=-2 \pi \sum_{k=1}^{\infty} \frac{\cos (2 \pi k d / h)}{\cos \left(\pi^{2} k / h\right)} .
$$

Taking into account a pole singularity

(Kress and Martensen 1970) consider the trapezoidal rule for Cauchy principal value integrals of the form

$$
\int_{-\infty}^{\infty} \frac{f(x)}{x-\xi} \mathrm{d} x, \quad \xi \in \mathbb{R}
$$


with $f \in H_{a}$ for some $a>0$. Even more interesting is a generalization of this taking $\xi$ to be a complex number inside the strip $G_{a}$. The integral is no longer interpreted as a Cauchy principal value. The pole at $x=\xi$ gives an extra residue term in the right-hand side of (3.38).

A standard integral for this case is the Hilbert transform of the Gaussian (in physics, the plasma dispersion function)

$$
w(z)=\frac{1}{\pi i} \int_{-\infty}^{\infty} \frac{e^{-t^{2}}}{t-z} \mathrm{~d} t, \quad \operatorname{Im} z>0,
$$

which is an entire function after analytic continuation and is related to the complementary error function. We have (see also (5.12))

$$
w(z)=e^{-z^{2}} \operatorname{erfc}(-i z), \quad z \in \mathbb{C} .
$$

(Matta and Reichel 1971) give several interesting examples for the complementary error function and related functions, such as the Fresnel integral and the Voigt functions. For the plasma dispersion function in (3.66) the trapezoidal rule becomes

$$
w(z)=\frac{h}{\pi i} \sum_{j=-\infty}^{\infty} \frac{e^{-(j h)^{2}}}{j h-z}+\theta \frac{2 e^{-z^{2}}}{1-e^{-2 \pi \mathrm{i} z / h}}+R(h),
$$

where $R(h)=\mathcal{O}\left(\exp \left(-\pi^{2} / h^{2}\right)(c f .(3.49))\right.$ and

$$
\theta=\left\{\begin{array}{ccc}
1 \quad \text { if } & \operatorname{Im} z<\frac{\pi}{h} \\
\frac{1}{2} & \text { if } & \operatorname{Im} z=\frac{\pi}{h} \\
0 & \text { if } & \operatorname{Im} z>\frac{\pi}{h}
\end{array}\right.
$$

To avoid numerical instability for the case that $z$ is close to $j h$ for some $j$, we can change $h$ or use a shifted rule. However, when we combine the particular $j$-term with the extra term in (3.68), the limit exists if $z \rightarrow j h$. For example, when $z \rightarrow 0$, we have

$$
\lim _{z \rightarrow 0}\left[-\frac{h}{\pi \mathrm{i} z}+\frac{2 e^{-z^{2}}}{1-e^{-2 \pi \mathrm{i} z / h}}\right]=1,
$$

which corresponds to $w(0)=1$ (this value follows from (3.67)).

For $\operatorname{Im} z<0$ we can use

$$
w(-z)=2 e^{-z^{2}}-w(z),
$$

although (3.68) also holds when $\operatorname{Im} z \leq 0$.

By using (3.68) and by taking into account the combination of singular 
terms or a proper choice of $h$, a uniform numerical algorithm for the function $w(z)$ and related functions can be constructed.

The function $w(z)$ is important in uniform asymptotic expansions of certain integrals when a pole and saddle point are close together; see Section 5.2, where the complementary error function is used in asymptotic approximations of the incomplete gamma functions.

\subsection{Contour integrals}

We discuss examples in which the starting point is a contour integral in the complex plane. We describe how simple transformations, inspired by the saddle point method for contour integrals, produce new integrals that are suitable for applying the trapezoidal rule on finite or infinite intervals.

First we compare two simple integrals

$$
F(\lambda)=\int_{-\infty}^{\infty} e^{-t^{2}+2 \mathrm{i} \lambda \sqrt{t^{2}+1}} \mathrm{~d} t
$$

and

$$
G(\lambda)=\int_{-\infty}^{\infty} e^{-t^{2}+2 i \lambda t} \mathrm{~d} t .
$$

When we compute $F(\lambda)$ by using Maple 9.5 with Digits $=10$, we obtain

$$
F(10)=-0.1837516481+0.5305342893 \mathrm{i} .
$$

With Digits $=40$, the answer is

$$
\begin{aligned}
F(10)= & -0.1837516480532069664418890663053408790017+ \\
& 0.5305342892550606876095028928250448740020 \mathrm{i} .
\end{aligned}
$$

So, the first answer seems to be correct in all shown digits.

The integral in (3.73) is slightly different and we repeat the computations for $G(\lambda)$. Maple 9.5, Digits $=10$, for $\lambda=10$, gives

$$
G(10)=-0.1257674520_{10}{ }^{-15} .
$$

With Digits $=40$, the answer is

$$
G(10)=0.16_{10}{ }^{-43} .
$$

The correct answer is

$$
G(\lambda)=\sqrt{\pi} e^{-\lambda^{2}},
$$

and for $\lambda=10$ we have

$$
G(10)=0.6593662989_{10}{ }^{-43} .
$$

Maple can evaluate the exact answer, but we forced Maple to use numerical quadrature to produce (3.76) and (3.77). 
We also used Mathematica to compute $G(10)$ and we received the message

NIntegrate failed to converge to prescribed accuracy after 7 recursive bisections in $t$ near $t=2.9384615384615387$.

Obtaining no result is better than obtaining a completely wrong result.

The lesson is: One should have some feeling about the computed result when dealing with oscillatory integrals, otherwise a completely incorrect answer can be accepted. And $\lambda=10$ is not really large.

From some points of view the integrals $F(\lambda)$ and $G(\lambda)$ are quite different, but an occasional user may not see this difference. The fact that $G \lambda$ ) is so small compared with $F(\lambda)$ can be explained by observing that the "phase function" of $F(\lambda)$, that is, $-t^{2}+2 \mathrm{i} \lambda \sqrt{t^{2}+1}$ has a stationary point (saddle point) inside the domain of integration (at $t=0$ ), whereas the saddle point of $G(\lambda)$ (at $t=\mathrm{i} \lambda$ ) is outside the domain.

By shifting the path upwards in the complex plane, through the saddle point at $t=\mathrm{i} \lambda$, or by just substituting $t=s+\mathrm{i} \lambda$, we obtain

$$
G(\lambda)=e^{-\lambda^{2}} \int_{-\infty}^{\infty} e^{-s^{2}} \mathrm{~d} s .
$$

Now we have the dominant factor in front of the integral, and the integral (in this case we know its answer) can be calculated easily by numerical quadrature, say, by using the trapezoidal rule.

This procedure will be carried out in several less trivial cases in which a given integral is transformed by selecting a new contour in the complex plane such that, for large values of parameters, a dominant factor is taken in front of the new integral. We use the standard methods of asymptotic analysis (saddle point methods) of which details can be found in (Wong 2001).

The evaluation of contour integrals is also important in the numerical inversion of Laplace transforms, when the initial vertical line of integration can be deformed into the left half-plane (Talbot 1979, Murli and Rizzardi 1990, Rizzardi 1995, Trefethen, Weideman and Schmelzer 2005, Weideman 2005).

Example 3.17. (The reciprocal gamma functions)

The Maclaurin series of the exponential function, $e^{w}=\sum_{n=0}^{\infty} w^{n} / n$ ! implies the Cauchy integral

$$
\frac{1}{n !}=\frac{1}{2 \pi \mathrm{i}} \oint \frac{e^{s}}{s^{n+1}} \mathrm{~d} s,
$$

where the integral can be taken over a circle in the complex plane. A more general form of this integral is Hankel's loop integral for the reciprocal gamma function:

$$
\frac{1}{\Gamma(z)}=\frac{1}{2 \pi \mathrm{i}} \int_{\mathcal{L}} \frac{e^{s}}{s^{z}} \mathrm{~d} s,
$$




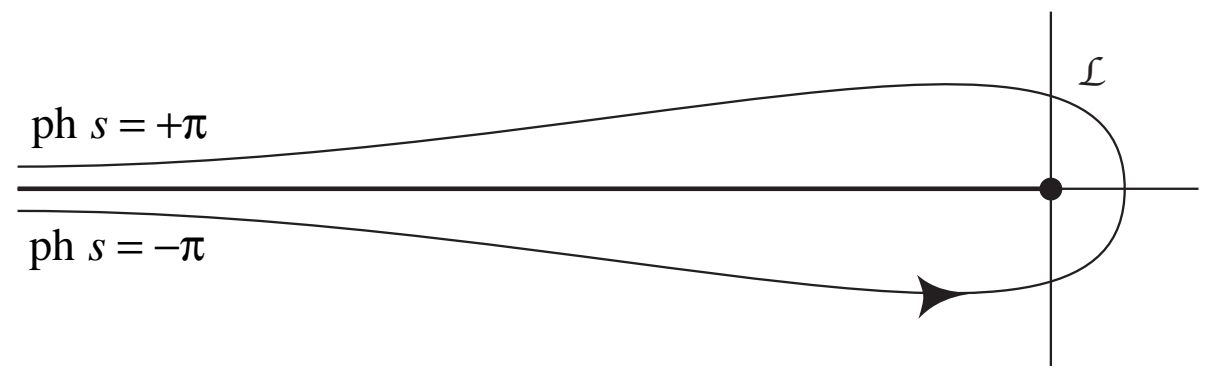

Figure 3.2. Hankel contour for the integral in (3.82).

where $\mathcal{L}$ is a contour as shown in Figure 3.2. It runs from $-\infty$ (with ph $s=$ $-\pi$ ), encircles the origin in the positive direction, and terminates at $s-\infty$, now with ph $s=+\pi$. The many-valued function $s^{-z}$ is assumed to be equal to 1 at $s=1$.

We consider $z>0$ and substitute $s=z t$ and obtain

$$
\frac{1}{\Gamma(z)}=\frac{e^{z} z^{1-z}}{2 \pi \mathrm{i}} \int_{\mathcal{L}} e^{z \phi(t)} \mathrm{d} t, \quad \phi(t)=t-1-\log t,
$$

where the contour is the same as in Figure 3.2. Compare this with (3.3). We choose a special contour on which the imaginary part of $\phi(t)=C$, where $C$ is a constant. In addition, we take this contour through the saddle point of the integrand at $t=1$, where $\phi^{\prime}(t)=0$. Since $\phi(1)=0$ we have $C=0$. Writing $t=r e^{\mathrm{i} \theta}$, we find that the equation $\operatorname{Im} \phi(t)=r \sin \theta-\theta=0$ is satisfied when the polar coordinates of $t$ satisfy

$$
r=\frac{\theta}{\sin \theta}, \quad-\pi<\theta<\pi .
$$

On this path, the steepest descent path, the the function $\phi(t)$ of $(3.83)$ is real and negative. The standard way to deal with integrals of this form in asymptotics is the transformation

$$
\phi(t)=-\frac{1}{2} w^{2},
$$

in which the lower branch of the path corresponds to $w<0$ and the upper part to $w>0$. Locally at $t=1$ we have

$$
w=\mathrm{i}(t-1)\left[1-\frac{1}{3}(t-1)+\cdots\right] .
$$

The transformation (3.85) gives

$$
\frac{1}{\Gamma(z)}=\frac{e^{z} z^{1-z}}{2 \pi} \int_{-\infty}^{\infty} e^{-\frac{1}{2} z w^{2}} g(w) \mathrm{d} w
$$


where

$$
g(w)=\frac{1}{\mathrm{i}} \frac{\mathrm{d} t}{\mathrm{~d} w}=\frac{t w}{\mathrm{i}(1-t)}=1+\frac{2}{3} \mathrm{i} w-\frac{1}{12} w^{2}+\cdots .
$$

The integrand in (3.87) is of the type (3.46), and we like to know the singularities of $g$ in order to determine the strip $G_{a}$ of Definition 3.9 around the real axis. The conformal mapping defined in (3.85) is regular at $t=1$, but $t=\exp (2 \pi \mathrm{i} n), n= \pm 1, \pm 2, \ldots$, which are important because of the manyvalued logarithm in $\phi(t)$, give singular points. These points correspond to $w$-values of which those with $n= \pm 1$ are closest to the real $w$-axis. The singularities that define the strip $G_{a}$ satisfy $\frac{1}{2} w^{2}= \pm 2 \pi \mathrm{i}$, which have imaginary parts equal to $\sqrt{2 \pi} \doteq 2.51$. We also see that the series in (3.88) has radius of convergence $2 \sqrt{\pi} \doteq 3.54$

In Table 3.3 we give details on the computation of $1 / \Gamma(z)$ by using (3.87) and (3.38) with $d=0$ and $d=\frac{1}{2} h$. We have chosen (see Remark 3.12) $h=\pi / \sqrt{\omega \log (1 / \varepsilon)}, \omega=\frac{1}{2} z$ with $\varepsilon=10^{-10}$, and sum the series for $j=$ $-j_{0},-j_{0}+1, \ldots, j_{0}-1, j_{0}$, where $j_{0}$ follows from the smallest $j$ that satisfies $\exp \left(-\omega j^{2} h^{2}\right)<\varepsilon$. For all $z$-values, $j_{0}=8$ (as explained in Remark 3.12, $j_{0}$ will not depend on $z$ ). We observe that the values of the relative errors $R_{0}(h) \Gamma(z)-1$ and $R_{h / 2}(h) \Gamma(z)-1$ are rather uniform for the values of $z$ shown, and that they have opposite signs. The sum of these errors shows that they are nearly equal in modulus. Computations are done in Maple with Digits $=15$.

Remark 3.18. The representation in (3.87) can also be used when $z$ is complex. When $|\mathrm{ph} z|<\frac{1}{2} \pi$ the integral remains convergent, and the exponential function $\exp \left(-\frac{1}{2} z w^{2}\right)$ is no longer real on the path. By turning the path of integration over an angle less than $\frac{1}{4} \pi$ we can repair this. We can even control convergence for $|\mathrm{ph} z|<\pi$. However, when we turn the path of integration, the singularities of $g$ mentioned earlier approach the real axis, and this makes the trapezoidal rule less efficient.

Because $t$, as function of $w$, is not explicitly known in terms of standard functions, the function $g$ of (3.88) needs an inversion procedure to compute the complex number $t$, when $w \in \mathbb{R}$ is given. In terms of the Lambert $W$-function, which is the inverse function of $W e^{W}$, we can write $t(w)=$ $-W\left(-\exp \left(\frac{1}{2} w^{2}-1\right)\right)$.

It may be of interest to use a different representation, in which such inversion is not needed. This can be found as follows.

When we use this parametrization of the contour and integrate with respect to $\theta$, we have

$$
\mathrm{d} t=\frac{\mathrm{d}\left(r e^{\mathrm{i} \theta}\right)}{\mathrm{d} \theta} \mathrm{d} \theta=[\mathrm{i}+h(\theta)] \mathrm{d} \theta
$$


Table 3.3. Values of $h$ and the remainders in the trapezoidal rule for the reciprocal gamma function $1 / \Gamma(z)$ by using (3.87) and (3.38). The last column gives the sum of the relative errors to show that the remainders are almost equal with opposite sign.

\begin{tabular}{||r|r|c|c|c}
\hline$z$ & $h$ & rel. error, $d=0$ & rel. error, $d=\frac{1}{2} h$ & sum of rel. errors \\
\hline 1 & 0.926 & $-0.41_{10}(-7)$ & $0.41_{10}(-7)$ & $0.23_{10}(-13)$ \\
2 & 0.656 & $0.46_{10}(-9)$ & $-0.46_{10}(-9)$ & $-0.39_{0}(-14)$ \\
3 & 0.536 & $0.44_{10}(-9)$ & $-0.44_{10}(-9)$ & $0.69_{0}(-14)$ \\
4 & 0.466 & $0.39_{10}(-9)$ & $-0.39_{0}(-9)$ & $0.29_{0}(-14)$ \\
5 & 0.415 & $0.36_{10}(-9)$ & $-0.36_{10}(-9)$ & $0.18_{0}(-13)$ \\
6 & 0.376 & $0.33_{10}(-9)$ & $-0.33_{10}(-9)$ & $0.11_{10}(-13)$ \\
7 & 0.350 & $0.31_{10}(-9)$ & $-0.31_{10}(-9)$ & $-0.25_{10}(-13)$ \\
8 & 0.327 & $0.39_{0}(-9)$ & $-0.39_{0}(-9)$ & $0.19_{0}(-13)$ \\
9 & 0.309 & $0.29_{10}(-9)$ & $-0.29_{0}(-9)$ & $-0.18_{10}(-13)$ \\
10 & 0.293 & $0.28_{10}(-9)$ & $-0.28_{0}(-9)$ & $-0.55_{10}(-13)$ \\
\hline
\end{tabular}

where $h(\theta)=(\cos \theta \sin \theta-\theta) / \sin ^{2} \theta$ is an odd function of $\theta$. It follows that

$$
\frac{1}{\Gamma(z)}=\frac{e^{z} z^{1-z}}{2 \pi} \int_{-\pi}^{\pi} e^{-z \Phi(\theta)} \mathrm{d} \theta
$$

where

$$
\Phi(\theta)=-\operatorname{Re} \phi(t)=1-\theta \cot \theta+\log \frac{\theta}{\sin \theta} .
$$

To evaluate $\Phi(\theta)$ for small values of $\theta$ we have

$$
\Phi(\theta)=\frac{1}{2} \theta^{2}+\frac{1}{36} \theta^{4}+\frac{1}{405} \theta^{6}+\frac{1}{4200} \theta^{8}+\frac{1}{42525} \theta^{10}+\cdots .
$$

All terms in the series are positive, as follows from the general form

$$
\Phi(\theta)=\sum_{n=1}^{\infty}(-1)^{n+1} \frac{2 n+1}{2 n} \frac{2^{2 n} B_{2 n}}{(2 n) !} \theta^{2 n}, \quad|\theta|<\pi,
$$

where $B_{2 n}$ are the Bernoulli numbers, and $(-1)^{n+1} B_{2 n}>0$.

The integrand belongs to $C_{p}^{\infty}([-\pi, \pi])$ (see Definition 3.1), with vanishing derivatives of all orders at the end points $\pm \pi$. 


\section{Example 3.19. (Bessel functions $J_{\nu}(x)$ )}

In Example 3.3 we have shown that for the Bessel function $J_{0}(x)$ a very simple integral can be used to apply the trapezoidal rule. For general integer values we can use the more general integral in (3.1), but when $n$ is large the many oscillations make the representations very unstable. In the present example we consider an integral representation that can be used to compute $J_{\nu}(x)$ for $\nu \geq x$, and large values of the parameters cause no problems.

We use the contour integral

$$
J_{\nu}(x)=\frac{1}{2 \pi \mathrm{i}} \int_{\mathcal{C}} e^{x \sinh t-\nu t} \mathrm{~d} t,
$$

where $\mathcal{C}$ starts at $\infty-\mathrm{i} \pi$ and terminates at $\infty+\mathrm{i} \pi$; see (Temme 1996, p. 222 ). On this contour oscillations will occur, but we will select a special contour that is free of oscillations for the case $x \leq \nu$.

We write

$$
\nu=x \cosh \mu, \quad \mu \geq 0 .
$$

The saddle point of

$$
x \sinh t-\nu t=x(\sinh t-t \cosh \mu)
$$

occurs at $t=\mu$, and at this saddle point the imaginary part of $x \sinh t-\nu t$ equals zero.

A path free of oscillations (a steepest descent path through the saddle point) can be described by the equation

$$
\operatorname{Im}(x \sinh t-\nu t)=0 .
$$

Writing $t=\sigma+\mathrm{i} \tau$ we obtain for the path the equation

$$
\cosh \sigma=\cosh \mu \frac{\tau}{\sin \tau}, \quad-\pi<\tau<\pi,
$$

and on this path we have

$$
\operatorname{Re}(x \sinh t-\nu t)=x(\sinh \sigma \cos \tau-\sigma \cosh \mu) .
$$

Integrating with respect to $\tau$, using $\mathrm{d} t / \mathrm{d} \tau=(\mathrm{d} \sigma / \mathrm{d} \tau+\mathrm{i}$ ) (where $\mathrm{d} \sigma / \mathrm{d} \tau$ is an odd function of $\tau$ ), we obtain

$$
J_{\nu}(x)=\frac{1}{2 \pi} \int_{-\pi}^{\pi} e^{x(\sinh \sigma \cos \tau-\sigma \cosh \mu)} \mathrm{d} \tau, \quad 0<x \leq \nu .
$$

The integrand is analytic and vanishes with all its derivatives at the end points of the interval $[-\pi, \pi]$.

When $\nu \gg x$ the Bessel function becomes very small and we can put the value of the integrand at $\tau=0$ as the dominant part in front of the integral. 
This gives the representation

$$
J_{\nu}(x)=\frac{e^{x(\sinh \mu-\mu \cosh \mu)}}{2 \pi} \int_{-\pi}^{\pi} e^{-x \psi(\tau)} \mathrm{d} \tau,
$$

where

$$
\psi(\tau)=\sinh \mu-\sinh \sigma \cos \tau+(\sigma-\mu) \cosh \mu .
$$

For small values of $\tau$ we have the expansion

$$
\psi(\tau)=\frac{1}{2} \sinh \mu \tau^{2}+\frac{2 \cosh ^{2} \mu+3}{72 \sinh \mu} \tau^{4}+\mathcal{O}\left(\tau^{6}\right) .
$$

We see that this breaks down when $\mu \rightarrow 0$, that is, $x \uparrow \nu$. This happens because the the phase function $x \sinh t-\nu t=x(\sinh t-t)$ (when $x=\nu$ ) has a cubic character at $t=0$. When $\mu=0$ the expansion of $\psi(\tau)$ reads

$$
\psi(\tau)=\frac{4 \sqrt{3}}{27} \tau^{3}+\frac{8 \sqrt{3}}{14175} \tau^{7}+\mathcal{O}\left(\tau^{9}\right) .
$$

In fact, the representation in (3.101) should not be used when $x$ and $\nu$ are nearly equal. For all $\mu>0$ the contour defined by (3.98) cuts the real $t$-axis under a right angle: when $\mu=0$ this angle is $\frac{1}{3} \pi$.

Remark 3.20. The change of behaviour of the integrand when $\nu \sim x$ is related to the turning point character of $J_{\nu}(x)$ when argument and order are equal. The function $\sqrt{z} J_{\nu}(\nu z)$ satisfies the differential equation

$$
w^{\prime \prime}+\left[\nu^{2}\left(\frac{z^{2}-1}{z^{2}}\right)-\frac{1}{4 z^{2}}\right] w=0 .
$$

In particular, when $\nu$ is large the solutions of this equation change behaviour from $z<1$ (monotonic character) to $z>1$ (oscillatory character). The Airy function is the main approximant in the large $\nu$ asymptotic approximation that holds in a neighbourhood of $z=1$. See (Olver 1997, Chapter 11).

To avoid this type of discontinuous behaviour of the contour we may choose a different path, which should run through the saddle point and end in the valleys when $t \rightarrow \infty \pm \mathrm{i} \pi$, but of which the parametrization does not become discontinuous when $\mu \downarrow 0$. For example, we can take

$$
\sigma=\mu+\frac{\tau^{2}}{\pi^{2}-\tau^{2}}, \quad-\pi<\tau<\pi .
$$

When using this path the imaginary part of the phase function $x \sinh t-\nu t$ will not vanish identically, but the oscillations of the integrand will have only a minor influence on the stability of the representation. That is, the function $\psi(\tau)$ in (3.101) will become different, but the dominant factor in front of the integral can still be extracted from the integrand. 
When $x>\nu$ (the oscillatory case), the Bessel function can be represented in a similar way, now by using two integrals (coming from the Hankel functions). First we consider

$$
H_{\nu}^{(1)}(x)=\frac{1}{\pi \mathrm{i}} \int_{-\infty}^{\infty+\pi \mathrm{i}} e^{x \sinh t-\nu t} \mathrm{~d} t
$$

now with $\nu=x \cos \mu, 0<\mu<\frac{1}{2} \pi$. The saddle points are now complex, and the relevant saddle point is $\mathrm{i} \mu$. The path of steepest descent follows from the equation

$$
\operatorname{Im}[\sinh (\sigma+\mathrm{i} \tau)-\cos \mu(\sigma+\mathrm{i} \tau)]=\sin \mu-\mu \cos \mu,
$$

which gives the equation

$$
\cosh \sigma=\frac{\tau \cos \mu+\sin \mu-\mu \cos \mu}{\sin \tau}, \quad 0<\tau<\pi,
$$

where $\sigma \leq 0$ when $\tau \leq \mu$ and $\sigma \geq 0$ when $\tau \geq \mu$. Using this path we obtain

$$
H_{\nu}^{(1)}(x)=\frac{e^{\mathrm{i} x(\sin \mu-\mu \cos \mu)}}{\pi \mathrm{i}} \int_{0}^{\pi} e^{-x \psi(\tau)} g(\tau) \mathrm{d} \tau,
$$

where

$$
\psi(\tau)=\sigma \cos \mu-\sinh \sigma \cos \tau, \quad g(\tau)=\frac{\mathrm{d} t}{\mathrm{~d} \tau}=\frac{\mathrm{d} \sigma}{\mathrm{d} \tau}+\mathrm{i} .
$$

After computing the Hankel function $H_{\nu}^{(1)}(x)$, the result for $J_{\nu}(x)$ follows from $J_{\nu}(x)=\operatorname{Re}\left[H_{\nu}^{(1)}(x)\right]$ (which holds when $x>0$ and $\nu \in \mathbb{R}$ ).

Example 3.21. (Modified Bessel functions $K_{\nu}(x)$ )

For the modified Bessel functions the representations are quite simple when we consider real parameters. In that case there is no turning point.

First consider the function (we take $x>0$ and $\nu \in \mathbb{R}$ )

$$
K_{\nu}(x)=\int_{0}^{\infty} e^{-x \cosh t} \cosh \nu t \mathrm{~d} t=\frac{1}{2} \int_{-\infty}^{\infty} e^{-x \cosh t+\nu t} \mathrm{~d} t .
$$

This integral has no oscillations and we can apply the trapezoidal rule if we wish without further steps. When the parameters are large it may be convenient to scale by extracting the dominant factor; we also like to have the peak at the origin, as in previous examples. We write

$$
\nu=x \sinh \mu,
$$

and see that the integrand of the second integral in (3.112) has a saddle point at $t=\mu$. We put $t=\mu+s$ and obtain

$$
K_{\nu}(x)=\frac{1}{2} e^{-x(\cosh \mu-\mu \sinh \mu)} \int_{-\infty}^{\infty} e^{-x \psi(s)} \mathrm{d} s
$$


where

$$
\psi(s)=\cosh \mu(\cosh s-1)+\sinh \mu(\sinh s-s) .
$$

By evaluating the Fourier integrals in (3.53), we obtain, for the remainder in

$$
K_{\nu}(x)=\frac{1}{2} h e^{-x(\cosh \mu-\mu \sinh \mu)} \sum_{j=-\infty}^{\infty} e^{-x \psi(j h)}+R_{0}(h),
$$

the series

$$
R_{0}(h)=-\sum_{\substack{m=-\infty \\ m \neq 0}}^{\infty} K_{\nu+2 \pi \mathrm{i} m / h}(x) .
$$

Example 3.22. (Modified Bessel functions $I_{\nu}(x)$ )

For the modified Bessel function $I_{\nu}(z)$ the starting point is

$$
I_{\nu}(x)=\frac{1}{2 \pi \mathrm{i}} \int_{\mathcal{C}} e^{x \cosh t-\nu t} \mathrm{~d} t,
$$

where the contour $\mathcal{C}$ starts at $\infty-\mathrm{i} \pi$ and ends at $\infty+\mathrm{i} \pi$. The relevant saddle point is $t=\mu$, where $\mu$ is as in (3.113). We write $t=\sigma+\mathrm{i} \tau$. The path of steepest descent through $t=\mu$ is given by $\operatorname{Im}[\cosh (\sigma+\mathrm{i} \tau)-\mu(\sigma+\mathrm{i} \tau)]=0$, which gives the parametrization

$$
\sinh \sigma=\frac{\mu \tau}{\sin \tau}, \quad-\pi<\tau<\pi
$$

We put the dominant exponential factor in front of the integral and obtain

$$
I_{\nu}(x)=\frac{e^{x(\cosh \mu-\mu \sinh \mu)}}{2 \pi} \int_{-\pi}^{\pi} e^{-x \psi(\tau)} \mathrm{d} \tau
$$

where

$$
\psi(\tau)=\cosh \mu-\cosh \sigma \cos \tau+(\sigma-\mu) \sinh \mu .
$$

For small values of $\tau$ we have

$$
\psi(\tau)=\frac{2 \mu^{2}+3+\mu \sinh \mu}{6 \cosh \mu} \tau^{2}+\mathcal{O}\left(\tau^{4}\right) .
$$

For $\nu=0$, that is, $\mu=0$, this representation reduces to

$$
I_{0}(x)=\frac{1}{2 \pi} \int_{-\pi}^{\pi} e^{x \cos \tau} \mathrm{d} \tau,
$$

which is a standard integral for this function (Abramowitz and Stegun 1964, Equation 9.6.16). 


\subsection{Some numerical aspects}

We discuss the main aspects in error handling of the trapezoidal rule (3.7) and (3.38) for the finite and infinite interval.

The size of the remainders $R_{n}^{d}$ and $R_{d}(h)$

In many cases where special functions are involved we can estimate these remainders. In the simple Examples 3.15 and 3.16, the series clearly show what happens. Also, in other cases we can compute or estimate the remainders, for example by evaluating the Fourier transforms needed in the series (3.52) or (3.53); see Example 3.21 where the remainder is given in (3.117) as an infinite sum of $K$-Bessel functions with complex order. For these functions asymptotic estimates are available.

The truncation error when taking the relevant terms of the series

This point is very relevant for infinite series. The smallest integer number $j_{0}$, such that in

$$
I(f)=\int_{-\infty}^{\infty} f(x) \mathrm{d} x=h\left[f(0)+2 \sum_{j=1}^{j_{0}} f(d+j h)\right]+S_{j_{0}},
$$

assuming that $f$ is even, $\left|S_{j_{0}}\right|$ or $\left|S_{j_{0}} / I(f)\right|$ is smaller than the desired accuracy $\varepsilon$, is called the number of relevant function evaluations corresponding to $h$ and $\varepsilon$.

In the finite case, as in Example 3.3, all terms in the sum (3.6) or (3.7) may be relevant, but sometimes the integrand in the finite integral becomes exponentially small at the end points (see (3.90)), and in that case the number of relevant function evaluations is important, and there is no need to compute all the terms in the finite sum.

How to choose h?

Another point is a stopping criterion when testing whether the number $h$ is small enough. When it is difficult to obtain useful and realistic analytic bounds for the remainders it may be necessary during computations to verify if the number $h$ is small enough. Let us denote the series in (3.38) by

$$
T_{d}(h)=h \sum_{j=-\infty}^{\infty} f(d+j h) .
$$

We can compare $T_{0}(h)$ and $T_{h / 2}(h)$ for a given $h$. When they agree within our desired accuracy, we are finished, and can take one of the two as our final result. However, as follows from Example 3.17 (see also Table 3.3), it 
is better to add both results, because

$$
T_{0}\left(\frac{1}{2} h\right)=\frac{1}{2}\left[T_{0}(h)+T_{h / 2}(h)\right],
$$

and the error in this result may be much smaller than the desired accuracy.

So, because of the fast convergence of the trapezoidal rule we may compute too many terms, and to control efficiency a different stopping criterion may be useful.

In the case of a finite interval the same fast convergence may occur, and to control both accuracy and efficiency a simple stopping criterion is a matter of trial and error for a certain problem.

In many problems that we have tested, in which, say, $N$ correct decimal digits had to be obtained, we have verified whether $h$ is sufficiently small to ensure that

$$
\left|T_{0}(h)-T_{h / 2}(h)\right|<\varepsilon, \quad \varepsilon=10^{-\frac{3}{4} N},
$$

and used (3.126) for the final result. In many cases this stopping criterion gave the results we wanted.

\subsection{Other aspects of numerical quadrature}

We mention other quadrature methods and conclude with further observations.

\section{Gauss quadrature}

Gauss quadrature is an efficient method for evaluating integrals. It is based on choosing the zeros of a class of orthogonal polynomials as the interpolation nodes. Usually, these zeros have to be precomputed, as well as the weights, which are also associated with the polynomials. This makes the method less attractive in adaptive algorithms, where we like to increase the number of nodes as often as we please. On the other hand, Gauss quadrature has proven to have its merits for certain types of integrals, and the underlying theory is very elegant. In a recent book, Gautschi (2004) the aspects of computation and approximation of orthogonal polynomials, and in particular Gauss quadrature rules, are discussed in great detail.

Filon's method for oscillatory integrals

In previous examples we have discussed how to deal with integrals on contours in the complex plane and how, particularly when the parameters are large, strong oscillations can be handled by choosing appropriate contours through saddle points.

In this section we discuss another method of how to deal with oscillatory integrals. For further discussions we refer to (Blakemore, Evans and Hyslop 1976), where a number of related methods for the evaluation of oscillatory integrals over infinite ranges are compared. 
Oscillatory integrals of the form

$$
I(f ; p)=\int_{a}^{b} f(x) e^{\mathrm{i} p x} \mathrm{~d} x
$$

can be evaluated using Filon's method (Filon 1928). The method, especially useful when $p$ is large, is based on the piecewise approximation of $f(x)$ on the interval of integration by low-degree polynomials. We give the following details.

The interval of integration $[a, b]$ is divided into $2 N$ equally spaced subintervals

$$
a=x_{0}<x_{1}<\cdots<x_{2 N}=b, \quad x_{k}=a+h k, \quad k=\frac{b-a}{2 N} .
$$

On each subinterval $\left[x_{2 k-2}, x_{2 k}\right]$ the function $f(x)$ is locally approximated by a polynomial $P_{k}(x)$ of degree at most 2 , and the corresponding integral on that interval

$$
\int_{2 k-2}^{2 k} P_{k}(x) e^{\mathrm{i} p x} \mathrm{~d} x
$$

is evaluated exactly. This gives

$$
I(f ; p) \approx h\left\{\mathrm{i} \alpha\left(e^{\mathrm{i} p a} f(a)-e^{\mathrm{i} p b} f(b)\right)+\beta E_{2 N}+\gamma E_{2 N-1}\right\},
$$

with

$$
\begin{aligned}
\theta^{3} \alpha & =\theta^{2}+\theta \sin \theta \cos \theta-2 \sin ^{2} \theta, \\
\theta^{3} \beta & =2\left[\theta\left(1+\cos ^{2} \theta\right)-2 \sin \theta \cos \theta\right], \\
\theta^{3} \gamma & =4[\sin \theta-\theta \cos \theta],
\end{aligned}
$$

and

$$
E_{2 N}=\sum_{k=0}^{N} \prime \prime f\left(x_{2 k}\right) e^{\mathrm{i} w x_{2 k}}, \quad E_{2 N-1}=\sum_{k=1}^{N} f\left(x_{2 k-1}\right) e^{\mathrm{i} w x_{2 k-1}} .
$$

The double prime over the first summation indicates that the first and last terms are to be multiplied by $\frac{1}{2}$.

The quantities $\alpha, \beta$, and $\gamma$ defined in (3.132) need to be recomputed when we change $h$, or for different $p$. When $\theta$ is small the right-hand sides in (3.132) should be expanded in powers of $\theta$ to preserve accuracy. We have

$$
\begin{aligned}
& \alpha=\frac{2}{45} \theta^{3}-\frac{2}{315} \theta^{5}+\frac{2}{4725} \theta^{7}+\cdots, \\
& \beta=\frac{2}{3}+\frac{2}{15} \theta^{2}-\frac{4}{105} \theta^{4}+\frac{2}{567} \theta^{6}+\cdots, \\
& \gamma=\frac{4}{3}-\frac{2}{15} \theta^{2}+\frac{1}{210} \theta^{4}-\frac{1}{11340} \theta^{6}+\cdots .
\end{aligned}
$$


It can be easily verified that for $p=0$, that is, $\theta=0$, Filon's method becomes Simpson's extended rule. For this quadrature rule we refer to (Abramowitz and Stegun 1964, Equation 25.4.6).

For recent investigations of Filon-type quadrature in connection with highly oscillatory integrals with extensive numerical and asymptotic analysis, see (Iserles 2004, Iserles 2005, Iserles and Nørsett 2005).

\section{Asymptotic expansion}

We consider integrals of the form

$$
I(f ; p)=\int_{0}^{\infty} f(x) e^{\mathrm{i} p x} \mathrm{~d} x,
$$

in which $p$ may be a complex parameter, and $f$ is a function sufficiently smooth on $(0, \infty)$ and with sufficient decay at $\infty$ to ensure convergence of the integral.

First we make some observations concerning integrals of the type (3.135). Earlier we gave the details of Filon's method, which can be used on a finite interval.

When derivatives of $f$ are available and when $p$ is large we can first integrate by parts to obtain the main contribution to the integral. In this way,

$$
I(f ; p)=\frac{\mathrm{i}}{p} f(0)-\frac{1}{p^{2}} f^{\prime}(0)-\frac{1}{p^{2}} \int_{0}^{\infty} f^{\prime \prime}(x) e^{\mathrm{i} p x} \mathrm{~d} x,
$$

which may be continued in order to obtain higher approximations, as far as the smoothness and growth conditions of the derivatives of $f$ allow.

\section{Odd or even functions}

When $f$ is an even function, all its odd derivatives at the origin vanish; hence, all terms with even powers of $p^{-1}$ vanish when we continue the expansion in (3.136). For example, when we take $f(x)=1 /\left(1+x^{2}\right)$, we have, when $p>0$,

$$
I(f ; p)=\int_{0}^{\infty} \frac{e^{\mathrm{i} p x}}{1+x^{2}} \mathrm{~d} x=\frac{1}{2} \pi e^{-p}+\mathrm{i} \int_{0}^{\infty} \frac{\sin (p x)}{1+x^{2}} \mathrm{~d} x,
$$

in which the real part of $I(f ; p)$ is exponentially small and the imaginary part is $\mathcal{O}\left(p^{-1}\right)$ when $p$ is large. So, computing $I(f ; p)$ of $(3.135)$ by using a quadrature rule when $f$ is a real even function may give a large relative error (but a small absolute error) in the real part of $I(f ; p)$, and similarly when $f$ is an odd function. 


\section{Analytic functions}

When $f$ is slowly decreasing at $\infty$, convergence of a quadrature rule may be rather poor. When $f$ is analytic in the right half -plane, and $p$ is positive, we may investigate if turning the path of integration up into the complex plane is possible. In that case convergence of the integral and of the quadrature rule may be improved.

\section{Orthogonal polynomials}

For integrals of the form

$$
I(f ; p)=\int_{-1}^{1} e^{\mathrm{i} p x} f(x) \mathrm{d} x,
$$

we can try to expand $f(x)$ in terms of Legendre polynomials, that is,

$$
f(x)=\sum_{n=0}^{\infty} c_{n} P_{n}(x),
$$

and obtain

$$
I(f ; p)=\sqrt{\frac{2 \pi}{p}} \sum_{n=0}^{\infty}(-\mathrm{i})^{n} c_{n} J_{n+\frac{1}{2}}(p),
$$

in terms of spherical Bessel functions; see (Temme 1996, Equation (6.64)).

In the same manner we can expand the function $f(x)$ in

$$
I(f ; p)=\int_{-1}^{1} e^{\mathrm{i} p x} \frac{f(x)}{\sqrt{1-x^{2}}} \mathrm{~d} x
$$

in terms of Chebyshev polynomials, $f(x)=\sum_{n=0}^{\infty} c_{n} T_{n}(x)$, and obtain

$$
I(f ; p)=\pi \sum_{n=0}^{\infty} \mathrm{i}^{n} c_{n} J_{n}(p),
$$

in terms of ordinary Bessel functions, which follows from (Temme 1996, Equation (9.20)).

For further examples on the use of orthogonal polynomials (also on infinite intervals), see (Patterson 1976/77).

\section{More general forms}

Oscillatory integrals also occur in the form

$$
I(f ; p)=\int_{0}^{\infty} f(x) \Phi(x p) \mathrm{d} x,
$$

where $\Phi$ is an oscillatory function. For example, in the case of Bessel functions we have the class of Hankel transforms 


$$
I_{\mu, \nu}(f ; p)=\int_{0}^{\infty} x^{\mu-1} f(x) J_{\nu}(p x) \mathrm{d} x,
$$

which play an important role in applied mathematics. In (Wong 1982) it is explained how quadrature rules of Gauss type can be constructed for these integrals and also for integrals of the type (3.135). In the latter case Wong gives a modification of the Gauss-Laguerre rule, and the method works for functions $f$ that are analytic in the right half-plane.

\section{Reducing the interval}

Because the exponential function in (3.135) is periodic, with interval of periodicity $[0,2 \pi / p]$, we can write $I(f ; p)$ in the form

$$
I(f ; p)=\frac{1}{p} \int_{0}^{2 \pi} e^{\mathrm{i} t} S_{p}(t) \mathrm{d} t, \quad S_{p}(t)=\sum_{k=0}^{\infty} f\left(\frac{2 \pi k+t}{p}\right) .
$$

\section{Another summation method}

When in (3.135) the exponential function is written in terms of the sine and cosine function, the resulting integrals can written as alternating series of positive and negative subintegrals that are computed individually (for example, when $f$ is positive). A similar method can also be used for (3.143) and (3.144) by using subintervals with endpoints the zeros of $\Phi(x)$ or $J_{\nu}(p x)$. See (Longman 1956).

Convergence acceleration schemes, for example Levin's or Weniger's transformations (see (Weniger 1989)), can be used when evaluating the series. For further information see (Clendenin 1966, Lyness 1985, Lucas and Stone 1995).

\subsection{Other curious exercises with integrals}

Needless to say, it will be evident that Maple and Mathematica are great tools when working with special functions, and in other areas of pure and applied mathematics. Numerical quadrature with these computer algebra packages can be very efficient, although, as mentioned at the beginning of Section 3.3, the user may not always get what he or she wants. We consider another example, a simple integral, where Maple and Mathematica give answers that are confusing and/or wrong (perhaps different results might have been obtained using later versions of Maple and Mathematica).

Consider

$$
F(u)=\int_{0}^{\infty} e^{u \mathrm{i} t} \frac{\mathrm{d} t}{t-1-\mathrm{i}}, \quad u>0 .
$$

Numerical quadrature gives $F(2)=-0.934349-0.70922$ i. 
Mathematica 4.1 gives for $u=2$, in terms of the Meijer G-function,

$$
F(2)=\pi G_{2,3}^{2,1}\left(\begin{array}{c}
0, \frac{1}{2} \\
0,0, \frac{1}{2}
\end{array} ; 2-2 \mathrm{i}\right) .
$$

For workers in special functions this may be a useful answer, but for those not familiar with this rather generalized hypergeometric function some confusion may arise. However, Mathematica can evaluate this answer numerically, and gives the result $F(2)=-0.547745-0.532287 \mathrm{i}$, which is not the same as that obtained earlier by using numerical quadrature.

Next, ask Mathematica to evaluate $F(u)$, that is, with a general argument. Surprisingly, the answer is much simpler:

$$
F(u)=e^{\mathrm{i} u-u} \Gamma(0, \mathrm{i} u-u),
$$

in terms of the incomplete gamma function. Again, asking Mathematica to evaluate numerically this result for $u=2$, we obtain $F(2)=-0.16114-$ $0.355355 \mathrm{i}$.

So, we have three numerical results:

$$
\begin{aligned}
& F_{1}=-0.934349-0.70922 \mathrm{i} \\
& F_{2}=-0.547745-0.532287 \mathrm{i}, \\
& F_{3}=-0.16114-0.355355 \mathrm{i} .
\end{aligned}
$$

Observe that $F_{2}=\left(F_{1}+F_{3}\right) / 2$. So, in some sense these answer have something in common. It turns out that $F_{1}$ is correct.

Turning to Maple 9.5, we obtain

$$
F(u)=e^{\mathrm{i} u-u} \operatorname{Ei}(1, \mathrm{i} u-u),
$$

in terms of the exponential integral. We can write this in terms of the incomplete gamma functions ${ }^{1}$ and obtain the same result (3.148) as Mathematica. This is the wrong answer.

Next, Maple 9.5, with $u=2$ :

$$
F(2)=e^{2 \mathrm{i}-2} \operatorname{Ei}(1,2 \mathrm{i}-2)+2 \pi \mathrm{i} e^{2 \mathrm{i}-2} .
$$

We see that the answer has an extra term now, and in Maple numerical evaluation gives

$$
F(2)=-0.9343493872-0.7092195102 \mathrm{i},
$$

which is the correct answer.

\footnotetext{
${ }^{1}$ Maple's convert function gives convert $(F(u), G A M M A)=e^{\mathrm{i} u-u} \Gamma(0, \mathrm{i} u-u)$; the notation $\operatorname{Ei}(a, z)$ is not widely used in the standard works for special functions. A better notation is $E_{a}(z)$, the generalized exponential integral.
} 
The extra term in (3.151) equals $2 \pi \mathrm{i}$ times the residue of the integrand of (3.146) at the pole $t=1+\mathrm{i}$. This residue arises when we turn the path of integration in (3.146) to the positive imaginary axis. The residue is missing in the answer $F_{3}$ of (3.149) and in $F_{2}$ it is taken into account incorrectly (only $\pi \mathrm{i}$ times the residue is added, which is why $F_{2}=\left(F_{1}+F_{3}\right) / 2$ ).

The confusing part is that both Maple and Mathematica also give different symbolic answers for $F(u)$ and $F(2)$.

\subsection{Numerical quadrature: Concluding remarks}

The first paper that mentioned the superiority of the trapezoidal rule for the integration of analytic functions on an infinite interval seems to be (Goodwin 1949). Many papers worked out this idea, of which we mention important contributions from the Japanese school: (Takahasi and Mori 1970, Takahasi and Mori 1971, Takahasi and Mori 1973/74) and Mori (1974). In these papers several transformations are discussed and error terms for a number of quadrature rules are represented as derivative-free contour integrals in the complex plane.

The trapezoidal rule may give the exact value of the integral when $h$ is less than some fixed value. For example (Rice 1973), if $m$ and $n$ are positive integers such that $m-n=0$, or $2,4, \ldots$, the integral

$$
\int_{-\infty}^{\infty} \frac{\sin ^{m} x}{x^{n}} \mathrm{~d} x
$$

is exactly integrated to the trapezoidal rule sum when $h<2 \pi / m$ ( $h$ can equal $2 \pi / m$ when $n \geq 2$ ). The proof follows from the fact that all of the terms in the series (3.53) for the remainder vanish. Rice gives several other interesting examples.

For further papers on the use of the trapezoidal rule on finite and infinite intervals, also with special transformations and interesting examples, we mention (Elliott 1998/99, Eggert and Lund 1989, Haber 1977, Squire 1976a, Squire 1976b, Weideman 2002).

\section{Recurrence relations}

Consider the recurrence relation

$$
y_{n+1}+a_{n} y_{n}+b_{n} y_{n-1}=0, \quad n=1,2,3, \ldots,
$$

where $a_{n}$ and $b_{n}$ are given, with $b_{n} \neq 0$. Many special functions of mathematical physics satisfy such a relation. Equation (4.1) is also called a linear homogeneous difference equation of the second order.

In analogy with the theory of differential equations, two linearly independent solutions $f_{n}, g_{n}$ exist in general, with the property that any solution $y_{n}$ 
of (4.1) can be written in the form

$$
y_{n}=A f_{n}+B g_{n},
$$

where $A$ and $B$ do not depend on $n$. We are interested in the special case that the pair $\left\{f_{n}, g_{n}\right\}$ satisfies

$$
\lim _{n \rightarrow \infty} \frac{f_{n}}{g_{n}}=0 .
$$

Then, for any solution (4.2) with $B \neq 0$, we have $f_{n} / y_{n} \rightarrow 0$ as $n \rightarrow \infty$. When $B=0$ in (4.2), we call $y_{n}$ a minimal solution; when $B \neq 0$, we call $y_{n}$ a dominant solution. When we have two initial values $y_{0}, y_{1}$, assuming that $f_{0}, f_{1}, g_{0}, g_{1}$ are known as well, then we can compute $A$ and $B$. That is,

$$
A=\frac{g_{1} y_{0}-g_{0} y_{1}}{f_{0} g_{1}-f_{1} g_{0}}, \quad B=\frac{y_{0} f_{1}-y_{1} f_{0}}{g_{0} f_{1}-g_{1} f_{0}} .
$$

The denominators are different from 0 when the solutions $f_{n}, g_{n}$ are linearly independent.

When we assume that the initial values $y_{0}, y_{1}$ are to be used for generating a dominant solution, then $A$ may, or may not, vanish; $B$ should not vanish: $y_{0} f_{1} \neq y_{1} f_{0}$. When, however, the initial values are to be used for the computation of a minimal solution, then the much stronger condition $y_{0} f_{1}=$ $y_{1} f_{0}$ should hold. It follows that, in this case, one and only one initial value can be prescribed; the other one follows from the relation $y_{0} f_{1}=y_{1} f_{0}$. In the numerical approach this leads to well-known instability phenomena for the computation of minimal solutions. The fact is that, when our initial values $y_{0}, y_{1}$ are not specified to an infinite precision, and consequently $B$ does not vanish exactly, the computed solution (4.2) always contains a fraction of $g_{n}$, the dominant solution. Hence, in the long run, our solution $y_{n}$ does not behave as a minimal solution, although we assumed that we were computing a minimal solution. This happens even if all further computations are done exactly.

In applications it is important to know whether a given equation (4.1) has dominant and minimal solutions. Often this can be easily concluded from the asymptotic behaviour of the coefficients $a_{n}$ and $b_{n}$. The following useful theorem is due to Perron and taken from (Gautschi 1967), which paper contains a wealth of information.

Theorem 4.1. (Perron) Assume that for large values of $n$ the coefficients $a_{n}, b_{n}$ behave as follows:

$$
a_{n} \sim a n^{\alpha}, \quad b_{n} \sim b n^{\beta}, \quad a b \neq 0,
$$

with $\alpha$ and $\beta$ real; assume that $t_{1}, t_{2}$ are the zeros of the characteristic polynomial $\Phi(t)=t^{2}+a t+b$ with $\left|t_{1}\right| \geq\left|t_{2}\right|$. 
[1] If $\alpha>\frac{1}{2} \beta$ then the difference equation (4.1) has two linearly independent solutions $y_{n, 1}$ and $y_{n, 2}$, with the property

$$
\frac{y_{n+1,1}}{y_{n, 1}} \sim-a n^{\alpha}, \quad \frac{y_{n+1,2}}{y_{n, 2}} \sim-\frac{b}{a n}^{\beta-\alpha}, \quad n \rightarrow \infty .
$$

[2] If $\alpha=\frac{1}{2} \beta$ then the difference equation (4.1) has two linearly independent solutions $y_{n, 1}$ and $y_{n, 2}$, with the property

$$
\frac{y_{n+1,1}}{y_{n, 1}} \sim t_{1} n^{\alpha}, \quad \frac{y_{n+1,2}}{y_{n, 2}} \sim t_{2} n^{\alpha}, \quad n \rightarrow \infty,
$$

assuming that $\left|t_{1}\right|>\left|t_{2}\right|$. If $\left|t_{1}\right|=\left|t_{2}\right|$ then we have

$$
\limsup _{n \rightarrow \infty}\left[\left|y_{n}\right|(n !)^{-\alpha}\right]^{\frac{1}{n}}=\left|t_{1}\right|
$$

for each non-trivial solution of (4.1).

[3] If $\alpha<\frac{1}{2} \beta$ then

$$
\limsup _{n \rightarrow \infty}\left[\left|y_{n}\right|(n !)^{-\beta / 2}\right]^{\frac{1}{n}}=\sqrt{|b|}
$$

for each non-trivial solution of (4.1).

Proof. For a proof we refer to the cited literature in (Gautschi 1967) or to (Elaydi 2005).

In case [1] and the first part of case [2] $f_{n}=y_{n, 2}$ is a minimal solution of (4.1). In addition, in the first part of [2],

$$
\lim _{n \rightarrow \infty} \frac{y_{n+1}}{n^{\alpha} y_{n}}=t_{r}, \quad r=1 \quad \text { or } \quad r=2,
$$

where $r=2$ holds for the minimal solution and $r=1$ for any other solution. To verify this, we derive from [1]:

$$
\frac{y_{n+1,2}}{y_{n+1,1}} / \frac{y_{n, 2}}{y_{n, 1}} \sim \frac{b}{a^{2}} n^{\beta-2 \alpha}, \quad n \rightarrow \infty .
$$

The right-hand side converges to 0 , since $\beta-2 \alpha<0$. It follows that $y_{n, 2} / y_{n, 1}$ converges to 0 . In the first part of [2] we have

$$
\frac{y_{n+1,2}}{y_{n+1,1}} / \frac{y_{n, 2}}{y_{n, 1}} \sim \frac{t_{2}}{t_{1}}, \quad n \rightarrow \infty .
$$

Since $\left|t_{1}\right|>\left|t_{2}\right|$ we again conclude that $y_{n, 2} / y_{n, 1}$ converges to 0 .

The second part of case [2] and case [3] of the theorem do not give information on the minimal and dominant solutions. As can be seen from the examples below we then need extra asymptotic information about the 
solutions of the difference equation (4.1). For the general asymptotic theory we refer to (Wong and Li 1992b) and (Wong and Li 1992a).

\section{Example 4.2. (Bessel and Legendre functions)}

We give the details of important recurrence relations for special functions and the stability aspects including the maximal and minimal solutions of the particular relation. The quantities $f_{n}, g_{n}$ denote the minimal and maximal solutions, respectively. For recent computer programs for Legendre functions and toroidal harmonics (a subclass of the Legendre functions) we refer to (Gil and Segura 1997, Gil and Segura 2000).

\section{(1) Bessel functions}

Recurrence relation:

$$
y_{\nu+1}-\frac{2 \nu}{z} y_{\nu}+y_{\nu-1}=0, \quad z \neq 0 .
$$

Solutions:

$$
f_{\nu}=J_{\nu}(z), \quad g_{\nu}=Y_{\nu}(z)
$$

This is covered by case [1] of the theorem, with

$$
a=-\frac{2}{z}, \quad \alpha=1, \quad b=1, \quad \beta=0 .
$$

Claim of the theorem:

$$
\frac{f_{\nu+1}}{f_{\nu}} \sim \frac{z}{2 \nu}, \quad \frac{g_{\nu+1}}{g_{\nu}} \sim \frac{2 \nu}{z} .
$$

Known asymptotic behaviour:

$$
f_{\nu} \sim \frac{1}{\sqrt{2 \pi \nu}}\left(\frac{e z}{2 \nu}\right)^{\nu}, \quad g_{\nu} \sim-\sqrt{\frac{2}{\pi \nu}}\left(\frac{e z}{2 \nu}\right)^{-\nu}, \quad \nu \rightarrow \infty .
$$

Similar results hold for the recurrence relation for the modified Bessel functions

$$
y_{\nu+1}+\frac{2 \nu}{z} y_{\nu}-y_{\nu-1}=0, \quad z \neq 0,
$$

with solutions $I_{\nu}(z)$ (minimal) and $e^{\pi \mathrm{i} \nu} K_{\nu}(z)$ (dominant).

(2) Legendre functions, recursion with respect to the order. Recurrence relation:

$$
y_{m+1}+\frac{2 m z}{\sqrt{z^{2}-1}} y_{m}+(m+\nu)(m-\nu-1) y_{m-1}=0 .
$$

Solutions:

$$
f_{m}=P_{\nu}^{m}(z), \quad g_{m}=Q_{\nu}^{m}(z)
$$

where

$$
\operatorname{Re} z>0, \quad \nu \in \mathbb{C} \quad \nu \neq-1,-2, \ldots, \quad z \notin(0,1] .
$$


This is covered by case [2] of the theorem, with

$$
\begin{gathered}
a=\frac{2 z}{\sqrt{z^{2}-1}}, \quad \alpha=1, \quad b=1, \quad \beta=2, \\
t_{1}=-\sqrt{\frac{z+1}{z-1}}, \quad t_{2}=\frac{1}{t_{1}}, \quad\left|t_{1}\right|>1>\left|t_{2}\right| .
\end{gathered}
$$

Claim of the theorem:

$$
\lim _{m \rightarrow \infty} \frac{f_{m+1}}{m f_{m}}=t_{2}, \quad \lim _{m \rightarrow \infty} \frac{g_{m+1}}{m g_{m}}=t_{1} .
$$

(3) Legendre functions, recursion with respect to the degree.

Recurrence relation:

$$
y_{n+1}-z \frac{2 n+2 \nu+1}{n+\nu-\mu+1} y_{n}+\frac{n+\nu+\mu}{n+\nu-\mu+1} y_{n-1}=0 .
$$

Solutions:

$$
f_{n}=Q_{\nu+n}^{\mu}(z), \quad g_{n}=P_{\nu+n}^{\mu}(z), \quad \operatorname{Re} z>0 .
$$

This is covered by case [2] of the theorem, with

$$
\begin{gathered}
a=-2 z, \quad \alpha=0, \quad b=1, \quad \beta=0, \\
t_{1}=z+\sqrt{z^{2}-1}, \quad t_{2}=\frac{1}{t_{1}}, \quad\left|t_{1}\right|>1>\left|t_{2}\right| .
\end{gathered}
$$

Claim of the theorem:

$$
\lim _{n \rightarrow \infty} \frac{f_{n+1}}{f_{n}}=t_{2}, \quad \lim _{n \rightarrow \infty} \frac{g_{n+1}}{g_{n}}=t_{1} .
$$

\subsection{Miller's algorithm}

From the previous discussion, it appears that the numerical computation of the minimal solution of a recurrence relation (4.1) with initial values $f_{0}$ and $f_{1}$ is quite problematic. One has to accept that the results are completely wrong after a few recursion steps. Of course, it depends on the required absolute or relative accuracy as to how much risk can be incurred, but in general one should be very careful.

From the asymptotic behaviour of the minimal and a dominant solution, one can usually conclude whether recursion for the minimal solution is dangerous.

We give details of an algorithm for computing a sequence of values

$$
f_{0}, f_{1}, \ldots, f_{N}
$$

of a minimal solution; $N$ is a non-negative integer. Obviously, we can apply 
(4.1) in the backward direction; in that case $f_{n}$ becomes a dominant solution and $g_{n}$ the minimal solution. Then we need two initial values $f_{N}$ and $f_{N-1}$. Miller's algorithm does not need these values, and uses a smart idea for the computation of the required sequence (4.30). The algorithm works for many interesting cases and gives an efficient method for computing the sequence (4.30).

Assume we have a relation of the form

$$
\sum_{n=0}^{\infty} \lambda_{n} f_{n}=s, \quad s \neq 0 .
$$

The series should be convergent and $\lambda_{n}$ and $s$ should be known. The series in (4.31) plays a role in normalizing the required minimal solution. The series may be finite; we only require that at least one coefficient $\lambda_{n}$ is different from zero. When just one coefficient, say $\lambda_{j}$, is different from zero, we assume that the value $f_{j}$ is available.

In Miller's algorithm a starting value $\nu$ is chosen, $\nu>N$, and a solution $\left\{y_{n}^{(\nu)}\right\}$ of (4.1) is computed with the false initial values

$$
y_{\nu+1}^{(\nu)}=0, \quad y_{\nu}^{(\nu)}=1 .
$$

The right-hand sides may be replaced by other values; at least one value should be different from zero. In some cases a judicious choice of these values may improve the convergence of the algorithm. The computed solution, with (4.32) as initial values, is a linear combination of the solutions $f_{n}$ and $g_{n}$ introduced earlier. A simple computation gives

$$
y_{n}^{(\nu)}=\frac{g_{\nu+1} f_{n}-f_{\nu+1} g_{n}}{g_{\nu+1} f_{\nu}-f_{\nu+1} g_{\nu}}, \quad n=0,1, \ldots, \nu+1 .
$$

This can be verified by checking the relations in (4.32). We write this in the form

$$
y_{n}^{(\nu)}=p_{\nu} f_{n}+q_{\nu} g_{n} .
$$

We observe that $y_{n}^{(\nu)} / p_{\nu}=f_{n}-\left[f_{\nu+1} / g_{\nu+1}\right] g_{n}$ and from (4.3) it follows that

$$
\lim _{\nu \rightarrow \infty} \frac{y_{n}^{(\nu)}}{p_{\nu}}=f_{n}, \quad 0 \leq n \leq N
$$

Apparently, when $\nu$ is large enough, an approximation of $f_{n}$ can be obtained from the quantities $y_{n}^{(\nu)}$ and $p_{\nu}$. However, in general, $p_{\nu}$ is not known. At this moment the normalizing relation (4.31) becomes of interest. We compute

$$
s^{(\nu)}=\sum_{n=0}^{\nu} \lambda_{n} y_{n}^{(\nu)}, \quad f_{n}^{(\nu)}=\frac{s}{s^{(\nu)}} y_{n}^{(\nu)} .
$$

Replacing $y_{n}^{(\nu)}$ in the series with $p_{\nu} f_{n}$, on account of (4.35), we then obtain 
$p_{\nu} \sim s^{(\nu)} / s$. It follows that we can consider $f_{n}^{(\nu)}$ as an approximation to $f_{n}$, if $\nu$ is large enough. That is, we assume that the circumstances are favourable, and that we can conclude that

$$
f_{n}=\lim _{\nu \rightarrow \infty} f_{n}^{(\nu)}, \quad n=0,1, \ldots, N .
$$

This claim will be founded by introducing extra conditions.

From (4.36) we obtain for the relative error (when $f_{n} \neq 0$ )

$$
\varepsilon_{n}^{(\nu)}=\frac{f_{n}^{(\nu)}-f_{n}}{f_{n}}=\frac{s / s^{(\nu)} y_{n}^{(\nu)}-f_{n}}{f_{n}}=\frac{s\left(p_{\nu}+q_{\nu} g_{n} / f_{n}\right)-s^{(\nu)}}{s^{(\nu)}} .
$$

We rewrite this in the form

$$
\varepsilon_{n}^{(\nu)}=\frac{\sigma_{\nu}-\rho_{\nu+1} / \rho_{n}+\tau_{\nu}}{1-\sigma_{\nu}-\tau_{\nu}}
$$

with

$$
\sigma_{\nu}=\frac{1}{s} \sum_{m=\nu+1}^{\infty} \lambda_{m} f_{m}, \quad \rho_{n}=\frac{f_{n}}{g_{n}}, \quad \tau_{\nu}=\frac{\rho_{\nu+1}}{s} \sum_{m=0}^{\nu} \lambda_{m} g_{m} .
$$

When introducing (4.31) we assumed that the series converges. Hence, $\sigma_{\nu} \rightarrow$ 0 as $\nu \rightarrow \infty$. Also (see (4.3)) we assumed that $\rho_{\nu} \rightarrow 0$. From this we infer that the relative error $\varepsilon_{n}^{(\nu)}$ of (4.39) converges to zero (as $\nu \rightarrow \infty$ ), if and only if $\tau_{\nu}$ converges to zero. Under this final condition, the limit in (4.37) holds.

For the numerical part of the method it is important to obtain an estimate of $\varepsilon_{n}^{(\nu)}$ for large values of $\nu$. In many cases it is not easy to obtain a strict estimate; usually some terms in (4.40) can be approximated by replacing the series with their dominant terms. Taking in the first series only the first term, and in the second series the final term, we obtain

$$
\sigma_{\nu} \simeq \frac{1}{s} \lambda_{\nu+1} f_{\nu+1}, \quad \tau_{\nu} \simeq \frac{1}{s} \rho_{\nu+1} \lambda_{\nu} g_{\nu}
$$

With these approximations (4.39) reads

$$
\begin{aligned}
\varepsilon_{n}^{(\nu)} & \simeq \frac{1}{s} \lambda_{\nu+1} f_{\nu+1}+\frac{f_{\nu+1}}{g_{\nu+1}} \frac{\lambda_{\nu} g_{\nu}}{s}-\frac{f_{\nu+1}}{g_{\nu+1}} \frac{g_{n}}{f_{n}} \\
& \simeq \frac{1}{s} \lambda_{\nu+1} f_{\nu+1}-\frac{f_{\nu+1}}{g_{\nu+1}} \frac{g_{n}}{f_{n}},
\end{aligned}
$$

since the second term on the first right-hand side is usually less important than the first term. A further step is to replace in this estimate $n$ by $N$, because, when the $N$-th element in the sequence in (4.30) is accurate, the situation will only improve for the remaining values. Reasoning in this way, 
we finally arrive at

$$
\varepsilon_{n}^{(\nu)} \simeq \frac{1}{s} \lambda_{\nu+1} f_{\nu+1}-\frac{f_{\nu+1}}{g_{\nu+1}} \frac{g_{N}}{f_{N}} .
$$

By using asymptotic estimates of the dominant and minimal solutions, the estimation of $\nu$ can be executed, perhaps numerically. The estimate of the error in (4.43) reflects two aspects of the algorithm for favourable convergence. The first term on the right-hand side of (4.43) indicates that the series in (4.31) should converge quickly. The second term indicates that the extent of dominance of $g_{n}$ with respect to $f_{n}$ is very significant.

In (Gautschi 1967) this algorithm is discussed in great detail (in a slightly different form). Gautschi estimates the starting point of the backward recursion by using asymptotic estimates of the special functions involved. In (Olver 1967) a direct numerical approach is used for obtaining a good starting point $\nu$. Olver also considers inhomogeneous recurrence equations. An excellent monograph for the numerical aspects of recurrence relations, including Miller's algorithm, is (Wimp 1984).

Example 4.3. (Computing $\boldsymbol{I}_{\boldsymbol{n}}(\boldsymbol{x})$ ) In (Bickley, Comrie, Miller, Sadler and Thompson 1952) the above method was introduced for computing the modified Bessel functions $I_{n}(x)$. The recurrence relation for these functions reads

$$
I_{n+1}(x)+\frac{2 n}{x} I_{n}(x)-I_{n-1}(x)=0 .
$$

A normalizing condition (4.31) is

$$
e^{x}=I_{0}(x)+2 I_{1}(x)+2 I_{2}(x)+2 I_{3}(x) \cdots .
$$

That is, $s=e^{x}, \lambda_{0}=1, \lambda_{n}=2(n \geq 1)$. We take $x=1$ and initial values (4.32) with $\nu=9$ and obtain the results given in Table 4.4.

The rightmost column in Table 4.4 is obtained by dividing the results of the middle column by (see (4.35) and (4.36))

$$
p_{9} \simeq \sum_{n=0}^{9} \lambda_{n} y_{n}^{(9)} / e^{1}=1.8071328986_{10}(+8) .
$$

When we take $N=5$, which means we want to compute the sequence $I_{0}(1), I_{1}(1), \ldots, I_{5}(1)$, we see that these quantities are computed with at least 10 correct decimal digits. For the present values of $x, \nu, N$ the estimate of the relative error $\varepsilon_{n}^{\nu}$ of (4.43) is $0.20_{10}^{-9}$, which is quite realistic.

\subsection{Recurrence relations and continued fractions}

For computing minimal solutions of three-term recurrence relations, the continued fraction for the ratios of consecutive solutions is also a useful tool. The basic result to be considered is Pincherle's theorem. 
Table 4.4. Computing the modified Bessel functions $I_{n}(x)$ for $x=1$ by using (4.44) in the backward direction. The underlined digits in the third column are correct.

\begin{tabular}{|c|c|c|}
\hline$n$ & $y_{n}$ before normalization & $y_{n} \doteq I_{n}(1)$ after normalization \\
\hline 0 & $2.287949300_{0}(+8)$ & $\underline{1.26606587801_{10}(-0)}$ \\
\hline 1 & $1.021317610_{0}(+8)$ & $\underline{5.65159104106_{10}(-1)}$ \\
\hline 2 & $2.453140800_{0}(+7)$ & $\underline{1.35747669794_{10}(-1)}$ \\
\hline 3 & $4.006129000_{0}(+6)$ & $\underline{2.216842492} 88_{10}(-2)$ \\
\hline 4 & $4.943400000_{0}(+5)$ & $\underline{2.73712022160_{0}(-3)}$ \\
\hline 5 & $4.905700000_{0}(+4)$ & $\underline{2.71463156012} 2_{0}(-4)$ \\
\hline 6 & $4.064000000_{0}(+3)$ & $\underline{2.2488661476} 61_{10}(-5)$ \\
\hline 7 & $2.890000000_{0}(+2)$ & $\underline{1.5992182} 9887_{10}(-6)$ \\
\hline 8 & $1.800000000_{0}(+1)$ & $\underline{9.96052919710_{0}(-8)}$ \\
\hline 9 & $1.000000000_{0}(+0)$ & 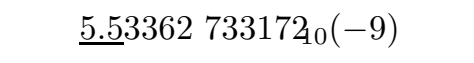 \\
\hline 10 & $0.000000000_{0}(+0)$ & $0.00000000009_{0}(-0)$ \\
\hline
\end{tabular}

Theorem 4.4. (Pincherle) Given a three-term recurrence relation

$$
y_{n+1}+b_{n} y_{n}+a_{n} y_{n-1}=0,
$$

then the continued fraction

$$
\frac{-a_{k}}{b_{k}+} \frac{-a_{k+1}}{b_{k+1}+} \cdots
$$

converges if and only if the recurrence relation possesses a minimal solution. Furthermore, if $f_{n}$ is a minimal solution, then the continued fraction converges to $f_{k} / f_{k-1}$.

Proof. See, for instance, (Gautschi 1967) and (Gil et al. 2007).

\subsection{The Gauss hypergeometric family}

Legendre functions are are already considered in Example 4.2 and are a special case of the Gauss hypergeometric functions, which are defined by the series given in $(2.19)$ for $|z|<1$. By using integral representations and connection formulas this function can be continued analytically on and outside the unit circle, with the general exception of the point $z=1$, which 
is usually a branch point. In this way the standard domain for this function is $|\operatorname{ph}(1-z)|<\pi$ with a branch cut from 1 to $+\infty$.

There are many formulas (contiguous relations) that connect a Gauss function with parameters $a, b, c$ to two other functions with parameters $a \pm$ $1, b \pm 1, c \pm 1$. Of special interest are the recurrence relations for the functions

$$
y_{n}(z)={ }_{2} F_{1}\left(\begin{array}{c}
a+\varepsilon_{1} n, b+\varepsilon_{2} n \\
c+\varepsilon_{3} n
\end{array} ; z\right)
$$

where $\varepsilon_{j}=0, \pm 1$ are fixed (not all $\varepsilon_{j}$ equal to zero). Each family $y_{n}$ satisfies a second order linear recurrence relation (difference equation) of the form

$$
A_{n} y_{n-1}+B_{n} y_{n}+C_{n} y_{n+1}=0 .
$$

All combinations of $\varepsilon_{j}$ in (4.49) constitute 26 cases of recursions but because of symmetry relations and functional relations for the Gauss functions, many of these 26 cases can be transformed into each other.

For example, we have the trivial symmetry relation

$$
{ }_{2} F_{1}\left(\begin{array}{c}
a, b \\
c
\end{array} ; z\right)={ }_{2} F_{1}\left(\begin{array}{c}
b, a \\
c
\end{array} ; z\right) .
$$

In addition, the following relations can be used (Abramowitz and Stegun 1964, p. 559)

$$
\begin{gathered}
{ }_{2} F_{1}\left(\begin{array}{c}
a, b \\
c
\end{array} ; z\right)=(1-z)^{-a}{ }_{2} F_{1}\left(\begin{array}{c}
a, c-b \\
c
\end{array} \frac{z}{z-1}\right), \\
{ }_{2} F_{1}\left(\begin{array}{c}
a, b \\
c
\end{array}\right)=(1-z)^{-b}{ }_{2} F_{1}\left(\begin{array}{c}
c-a, b \\
c
\end{array} \frac{z}{z-1}\right), \\
{ }_{2} F_{1}\left(\begin{array}{c}
a, b \\
c
\end{array}\right)=(1-z)^{c-a-b}{ }_{2} F_{1}\left(\begin{array}{c}
c-a, c-b \\
c
\end{array}\right) .
\end{gathered}
$$

By using these four relations it is possible to reduce the set of 26 recursions to just five cases from which the properties of the remaining 21 recursions can be obtained (by using other considerations we can eliminate one of these cases, but here we consider the five cases).

For each of the five basic recursions, the following points have to be considered.

(1) To find the domains in the $z$-plane where we can identify minimal and 
dominant solutions of the recurrence relation (luckily, these domains do not depend on $a, b$, or $c$ ). These domains follow from Theorem 4.1, and are defined by by the equation $\left|t_{1}\right|=\left|t_{2}\right|$, where $t_{1}$ and $t_{2}$ are the zeros of the characteristic polynomial.

(2) To identify a pair of linearly independent solutions of each recurrence in each domain. This means that we want to know which functions, next to ${ }_{2} F_{1}\left(a+\varepsilon_{1} n, b+\varepsilon_{2} n ; c+\varepsilon_{3} n ; z\right)$ for a given set $\varepsilon_{j}$, are solutions of the same recurrence relation, and which of these are minimal or dominant.

These points are considered in great detail in the recent papers by (Gil, Segura and Temme 2006a, Gil, Segura and Temme 2006b), of which the second paper contains the full proofs. It is quite remarkable that a systematic study of the set of recursions for the Gauss function has been neglected in the literature so far. Of course, an important subclass, the Legendre functions, was earlier (Gil and Segura 1997, Gil and Segura 2000); (Gautschi 1967) has paid attention to recursions for the incomplete beta functions, which are also a special case. Furthermore, (Wimp 1984) has discussed the Miller algorithm with some examples of Gauss hypergeometric functions.

For each of the five basic recursions the coefficients $A_{n}, B_{n}$ and $C_{n}$ of the recurrence relation (4.50) are available now (Gil et al. 2006a, Gil et al. 2006b). A nice property of these recurrences is that the ratios of the coefficients $A_{n} / C_{n}$ and $B_{n} / C_{n}$ have finite limits as $n \rightarrow+\infty$ except, perhaps, at some singular points in the $z$-plane. We use the following notation:

$$
\alpha=\lim _{n \rightarrow \infty} \frac{A_{n}}{C_{n}}, \quad \beta=\lim _{n \rightarrow \infty} \frac{B_{n}}{C_{n}} .
$$

According to Perron's theorem the existence of minimal solutions is guaranteed when the roots of the characteristic equation $t^{2}+\beta t+\alpha=0$ have different moduli. The equation $\left|t_{1}\right|=\left|t_{2}\right|$ gives curves in the complex $z$-plane, and these curves are the boundaries of domains where we can distinguish minimal and dominant solutions of the recurrence relations.

To denote the cases we use the notation $\left(\operatorname{sign}\left(\varepsilon_{1}\right) \operatorname{sign}\left(\varepsilon_{2}\right) \operatorname{sign}\left(\varepsilon_{3}\right)\right)$. For example, the recursion related to

$$
y_{n}={ }_{2} F_{1}\left(\begin{array}{c}
a+n, b \\
c+n
\end{array} ; z\right)
$$

will be denoted by $(+0+)$. By using $(4.53)$ we can write this as

$$
y_{n}=(1-z)_{2}^{-b} F_{1}\left(\begin{array}{c}
c-a, b \\
c+n
\end{array} ; \zeta\right), \quad \zeta=\frac{z}{z-1} .
$$

If we have a pair $\left\{F_{n}, G_{n}\right\}$ of minimal and dominant solutions of recursion 
related to the basic form $(00+)$, we can use the relation (4.53) to obtain a pair $\left\{f_{n}, g_{n}\right\}$ for the recursion related to $(+0+)$.

We give the details of two basic recursions, namely the cases $(+00)$ and $(++0)$. For the other basic forms $(++-),(+0-)$, and $(00+)$ we refer to (Gil et al. 2006a, Gil et al. 2006b).

We give six solutions of the recurrence relation for the two cases considered here. These solutions are related with the three pairs of Gauss functions that are linearly independent solutions in the neighbourhood of the singular points $0,1, \infty$ of the Gauss hypergeometric differential equation (2.20).

The $(+\quad 0 \quad 0)$ recursion

The recurrence relation reads

$$
A(a+n) y_{n-1}+B(a+n) y_{n}+C(a+n) y_{n+1}=0,
$$

where

$$
A(a)=c-a, \quad B(a)=2 a-c-(a-b) z, \quad C(a)=a(z-1) .
$$

The zeros of the characteristic polynomial of the recurrence relation (4.58) are

$$
t_{1}=1, \quad t_{2}=1 /(1-z) .
$$

The equation $\left|t_{1}\right|=\left|t_{2}\right|$ holds when $|1-z|=1$, which defines a circle with centre $z=1$ and radius 1 . Inside the circle we have $\left|t_{2}\right|>\left|t_{1}\right|$.

Solutions of the recurrence relation (4.58) are

$$
\begin{aligned}
& y_{1, n}={ }_{2} F_{1}\left(\begin{array}{c}
a+n, b ; z \\
c
\end{array}\right), \\
& y_{2, n}=\frac{\Gamma(a+n+1-c)}{\Gamma(a+n)}{ }_{2} F_{1}\left(\begin{array}{c}
1+a+n-c, 1+b-c ; z \\
2-c
\end{array}\right), \\
& y_{3, n}=\frac{\Gamma(a+n+1-c)}{\Gamma(a+b+n+1-c)}{ }_{2} F_{1}\left(\begin{array}{c}
a+n, b ; 1-z \\
a+b+n+1-c
\end{array}\right), \\
& y_{4, n}=(1-z)^{-n} \frac{\Gamma(n+a+b-c)}{\Gamma(n+a)}{ }_{2} F_{1}\left(\begin{array}{c}
c-a-n, c-b \\
c+1-a-b-n
\end{array} ; 1-z\right), \\
& y_{5, n}=(-z)^{-n} \frac{\Gamma(n+1-c+a)}{\Gamma(1+a+n-b)}{ }_{2} F_{1}\left(\begin{array}{c}
a+n, a+n+1-c \\
a+n+1-b
\end{array}\right), \\
& y_{6, n}=\frac{\Gamma(a+n-b)}{\Gamma(a+n)}{ }_{2} F_{1}\left(\begin{array}{c}
b, b+1-c \\
b+1-a-n
\end{array} ; \frac{1}{z}\right) .
\end{aligned}
$$


All these functions are solutions of the recurrence relation (4.59). Only one of these is the minimal solution. Any solution different from the minimal solution cannot be minimal, because the minimal solution is unique (up to multiplicative factors not depending on $n$ ).

In the following scheme we give the properties of the six solutions:

$\begin{array}{ccc} & |z-1|<1 & |z-1|>1 \\ y_{1} & \text { dominant } & \text { dominant } \\ y_{2} & \text { dominant } & \text { dominant } \\ y_{3} & \text { minimal } & \text { dominant } \\ y_{4} & \text { dominant/minimal } & \text { dominant } \\ y_{5} & \text { dominant } & \text { minimal } \\ y_{6} & \text { dominant } & \text { dominant } / \text { minimal }\end{array}$

where, when two possibilities appear, the first one corresponds to $n \rightarrow+\infty$ and the second one to $n \rightarrow-\infty$.

The $(++0)$ recursion

The recurrence relation reads

$$
A(a+n, b+n) y_{n-1}+B(a+n, b+n) y_{n}+C(a+n, b+n) y_{n+1}=0
$$

where

$$
\begin{aligned}
& A(a, b)=(c-a)(c-b)(c-a-b-1), \\
& B(a, b)=(c-a-b)\{c(a+b-c)+c-2 a b \\
& \quad+z[(a+b)(c-a-b)+2 a b+1-c]\}, \\
& C(a, b)=a b(c-a-b+1)(1-z)^{2} .
\end{aligned}
$$

The coefficients of characteristic equation $\lambda^{2}+\beta \lambda+\alpha=0$ are

$$
\alpha=1 /(1-z)^{2}, \quad \beta=-2(1+z) /(1-z)^{2},
$$

with roots

$$
t_{1}=\frac{1}{(1-\sqrt{z})^{2}}, \quad t_{2}=\frac{1}{(1+\sqrt{z})^{2}} .
$$

The equation $\left|t_{1}\right|=\left|t_{2}\right|$ holds when $z \leq 0$, otherwise $\left|t_{1}\right|>\left|t_{2}\right|$. In this case, the region $\left|t_{1}\right| \neq\left|t_{2}\right|$ is one connected region. 
We give six solutions of the recurrence relation (4.63):

$$
\begin{aligned}
& y_{1, n}={ }_{2} F_{1}\left(\begin{array}{c}
a+n, b+n \\
c
\end{array} ;\right), \\
& y_{2, n}=\frac{\Gamma(1+a-c+n) \Gamma(1+b-c+n)}{\Gamma(a+n) \Gamma(b+n)}{ }_{2} F_{1}\left(\begin{array}{c}
1+a-c+n, 1+b-c+n \\
2-c
\end{array} ; z\right), \\
& y_{3, n}=\frac{\Gamma(1+a-c+n) \Gamma(1+b-c+n)}{\Gamma(1+a+b-c+2 n)}{ }_{2} F_{1}\left(\begin{array}{c}
a+n, b+n \\
1+a+b-c+2 n
\end{array} ; 1-z\right), \\
& y_{4, n}=(1-z)^{-2 n} \frac{\Gamma(a+b-c+2 n)}{\Gamma(a+n) \Gamma(b+n)}{ }_{2} F_{1}\left(\begin{array}{c}
-a+c-n,-b+c-n \\
1-a-b+c-2 n
\end{array} ; 1-z\right), \\
& y_{5, n}=(-z)^{-n} \frac{\Gamma(1+a-c+n)}{\Gamma(b+n)}{ }_{2} F_{1}\left(\begin{array}{c}
a+n, 1+a-c+n \\
1+a-b
\end{array}\right), \\
& y_{6, n}=(-z)^{-n} \frac{\Gamma(1+b-c+n)}{\Gamma(a+n)}{ }_{2} F_{1}\left(\begin{array}{c}
b+n, 1+b-c+n \\
1-a+b
\end{array} ; \frac{1}{z}\right) .
\end{aligned}
$$

In the following scheme we give the properties of the six solutions; the properties hold in $\mathbb{C} \backslash\{z \leq 0\}$ :

$$
\begin{array}{cc}
y_{1, n} & \text { dominant } \\
y_{2, n} & \text { dominant } \\
y_{3, n} & \text { minimal/dominant } \\
y_{4, n} & \text { dominant/minimal } \\
y_{5, n} & \text { dominant } \\
y_{6, n} & \text { dominant }
\end{array}
$$

where, when two possibilities appear, the first one corresponds to $n \rightarrow+\infty$ and the second one to $n \rightarrow-\infty$.

This case has applications for Jacobi polynomials. We have

$$
P_{n}^{(\alpha, \beta)}(x)=\left(\begin{array}{c}
n+\alpha \\
n
\end{array}\right)\left(\frac{1+x}{2}\right)^{n}{ }_{2} F_{1}\left(\begin{array}{c}
-n,-\beta-n \\
\alpha+1
\end{array} ; z\right), \quad z=\frac{x-1}{x+1} .
$$

A representation with $+n$ at the $a$ and $b$ places follows from applying (4.54). The interval of orthogonality is $[-1,1]$, and if $x \in[-1,1]$ we have $z \leq 0$. We see that in and outside the orthogonality interval the Jacobi polynomial $P_{n}^{(\alpha, \beta)}(x)$ is a dominant solution of its recurrence relation and this relation can be used for computing these polynomials in the forward direction. Only the usual rounding errors should be taken into account. 
Remark 4.5. Observe that $y_{3, n}$ and $y_{4, n}$ are related to recursion schemes of the form $(++2+)$ and $(--2-)$, which fall outside the set of 26 forms considered here.

\subsection{Anomalous behaviour of some second-order homogeneous and first-order inhomogeneous recurrences}

It is usually assumed that asymptotic information is enough for predicting stable directions for recursion, at least for second order homogeneous equations. This, however, is not always true and there exist examples for which, for finite orders $n$, a minimal solution interchanges its role with certain dominant solutions. This, as a consequence, implies the anomalous convergence of the associated continued fraction to a value different from the ratio of consecutive minimal solutions, a phenomenon first observed (Gautschi 1977) in connection with a recurrence relation for confluent hypergeometric functions. See also (Deaño and Segura 2006, Gil et al. 2007).

\section{Uniform asymptotic expansions}

Writing efficient algorithms for special functions may become problematic when several large parameters are involved. In particular, problems arise when functions suddenly change their behaviour, say from monotonic to oscillatory behaviour. For example, the Bessel function $J_{\nu}(x)$ has a turning point at $x=\nu$ (see Remark 3.20), and for large $\nu$ the function is oscillatory for $x>\nu$ and monotonic for $x<\nu$. See Figure 5.3. To describe the asymptotic behaviour of $J_{\nu}(x)$ for large $\nu$ and $x \sim \nu$ we need the Airy function $\operatorname{Ai}(z)$, which is a solution of the simple turning point equation $w^{\prime \prime}-z w=0$.

For many other special functions of mathematical physics, powerful uniform asymptotic expansions are available, which describe precisely how the functions behave, which are valid for large domains of the parameters, and which provide tools for designing high-performance computational algorithms.

In this section we discuss examples of uniform asymptotic expansions and the numerical aspects. We explain why these expansions are useful, and why they are usually difficult to handle in numerical algorithms. First we consider uniform expansions of the incomplete gamma functions. These functions are important in probability theory, but also in physical problems. Another important class concerns the functions having a turning point in their defining differential equation, in which case Airy-type expansions arise, as mentioned earlier. We give details of Airy-type expansions for Bessel functions. 


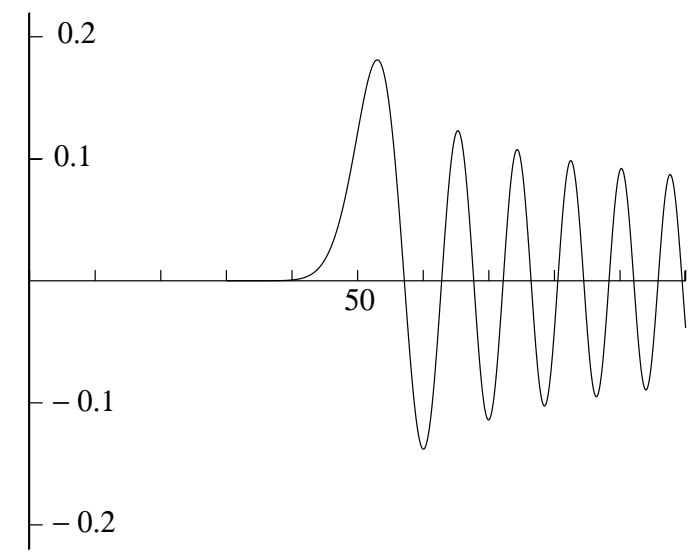

Figure 5.3. The Bessel function $J_{50}(x), 0 \leq x \leq 100$.

\subsection{Asymptotic expansions for the incomplete gamma functions}

We recall the definitions of the incomplete gamma functions

$$
\gamma(a, z)=\int_{0}^{z} t^{a-1} e^{-t} \mathrm{~d} t, \quad \Gamma(a, z)=\int_{z}^{\infty} t^{a-1} e^{-t} \mathrm{~d} t,
$$

where for the first integral we need $\operatorname{Re} a>0$ and for both integrals we assume that $|\mathrm{ph} z|<\pi$.

Integrating by parts in the second integral gives

$$
\Gamma(a, z)=-\int_{z}^{\infty} t^{a-1} \mathrm{~d} e^{-t}=z^{a-1} e^{-z}+(a-1) \int_{z}^{\infty} t^{a-2} e^{-t} \mathrm{~d} t .
$$

Repeating this we find for $n=1,2,3, \ldots$

$$
\begin{aligned}
\Gamma(a, z)=z^{a-1} e^{-z} & {\left[1+\frac{a-1}{z}+\frac{(a-1)(a-2)}{z^{2}}+\cdots\right.} \\
+ & \left.\frac{(a-1)(a-2) \cdots(a-n+1)}{z^{n-1}}\right]+R_{n}(a, z),
\end{aligned}
$$

where

$$
R_{n}(a, z)=(a-1)(a-2) \cdots(a-n) \int_{z}^{\infty} t^{a-n-1} e^{-t} \mathrm{~d} t .
$$

For positive $a$ and $z$ we can easily find a bound for the remainder. If $a>n+1$ the integrand has a maximum at $t_{0}=a-n-1$. If $a \leq n+1$ the integrand is decreasing on $t>0$. In any case, if $z>a-n$, we can integrate in the integral in (5.4) with respect to the variable $p=t+(n-a) \ln t$, which gives

$$
R_{n}(a, z)=(a-1)(a-2) \cdots(a-n) \int_{p_{0}}^{\infty} e^{-p} \frac{\mathrm{d} p}{t+n-a},
$$


where $p_{0}=z+(n-a) \ln z$. Because $t \geq z$ in (5.4) we have $t+n-a \geq z+n-a$, and we obtain

$$
R_{n}(a, z) \leq \frac{(a-1)(a-2) \cdots(a-n)}{z^{n}} \frac{z}{(z+n-a)} z^{a-1} e^{-z}, \quad z>a-n .
$$

This shows the asymptotic character of the expansion (5.3) when $n>a$, as $z \rightarrow \infty$. However, the condition $z>a-n$ is not enough to make it a useful expansion. When $a$ is also large, say $a \sim z$, then the ratios of successive terms in the expansion (5.3) are of order $\mathcal{O}(1)$, and, hence, the terms are not even becoming small. We say that the expansion in (5.3) does not hold uniformly with respect to $a>0$. However, it holds uniformly for $a$ in compact intervals.

For the function $\gamma(a, z)$ we can also obtain an asymptotic representation. Integration by parts now starts with

$$
\gamma(a, z)=\frac{1}{a} \int_{0}^{z} e^{-t} \mathrm{~d} t^{a}=\frac{1}{a} z^{a} e^{-z}+\frac{1}{a} \int_{0}^{z} t^{a} e^{-t} \mathrm{~d} t .
$$

This is the beginning of the convergent expansion

$$
\gamma(a, z)=\frac{1}{a} z^{a} e^{-z} \sum_{n=0}^{\infty} \frac{z^{n}}{(a+1)(a+2) \cdots(a+n)} .
$$

This expansion has an asymptotic character when $a$ is large, and again we see that the asymptotic property does not hold uniformly with respect to $z>0$ (although the expansion is convergent for all finite $z$ ). Both expansions in (5.3) and (5.8) have their limitations with respect to in which domains we can use them for numerical computations. But they share one nice property: the coefficients can be computed very easily.

\subsection{Uniform asymptotic expansions}

The asymptotic expansions of the incomplete gamma functions $\Gamma(a, z)$ and $\gamma(a, z)$ given in the previous section become useless when both parameters $a$ and $z$ are of the same size. The representation for $\Gamma(a, z)$ in $(5.3)$ is valid for any $a$ and $z$ (with the usual condition $|\operatorname{ph} z|<\pi$ ), but we can use it as an asymptotic representation only when $|z| \gg|a|$. As mentioned after (5.6), the ratio of successive terms in the representation (5.3) are of order $\mathcal{O}(1)$ when $z \sim a$.

We give details of the uniform asymptotic expansions for the incomplete gamma functions. One essential feature of such expansions is the role of certain special functions in the expansions. In the standard, non-uniform, expansions usually only elementary functions occur, such as exponential and trigonometric functions. In uniform expansions we usually need higher transcendental functions, such as Airy functions, error functions, Fresnel 


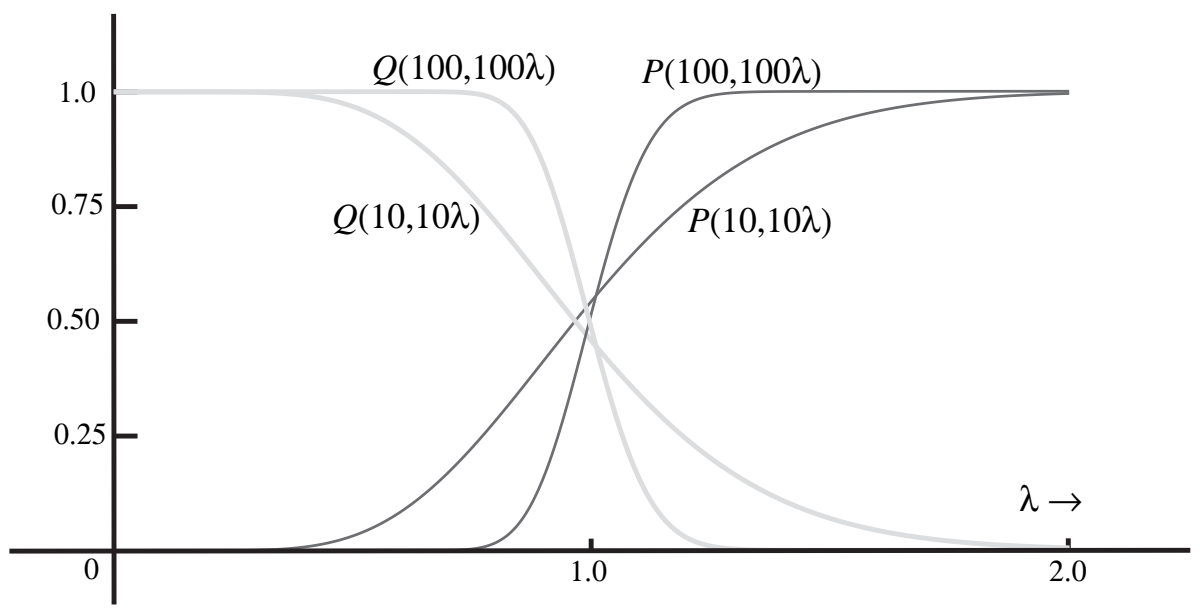

Figure 5.4. The function $P(a, \lambda a)$ and $Q(a, \lambda a)$ for $\lambda \in[0,2]$ and $a=10$ and $a=100$.

integrals, and so on. The proper choice of these special functions is not always clear without a further study of asymptotic analysis.

We consider a uniform expansion that can be used for both $\Gamma(a, z)$ and $\gamma(a, z)$, and for all large values of $a$ and $z$, as well as for complex values, but we continue the discussion for positive real parameters.

The incomplete gamma functions are related to cumulative distribution functions of probability theory, with the underlying distribution being the gamma distribution. In particular the incomplete gamma functions appear in the form of the chi-square probability functions. For several reasons it is convenient to work with the normalized functions

$$
P(a, z)=\frac{\gamma(a, z)}{\Gamma(a)}, \quad Q(a, z)=\frac{\Gamma(a, z)}{\Gamma(a)},
$$

for example, because of their role in probability theory, and because no overflow occurs for large values of $a$. We have

$$
P(a, z)+Q(a, z)=1 .
$$

In Figure 5.4 we show the graphs of these functions, where we have used a parameter $\lambda$ to scale the $z$-variable. In fact we give the graphs of the functions $P(a, \lambda a)$ and $Q(a, \lambda a)$ for $\lambda \in[0,2]$ and $a=10$ and $a=100$. As $a$ increases the graphs become steeper when $\lambda$ passes the value $\lambda=1$.

As is well known in the theory of cumulative distribution functions, many of these functions approach the normal or Gaussian probability functions when certain parameters become large. In probability theory the normal 
distribution functions are defined by

$$
P(z)=\frac{1}{\sqrt{2 \pi}} \int_{-\infty}^{z} e^{-\frac{1}{2} t^{2}} \mathrm{~d} t, \quad Q(z)=\frac{1}{\sqrt{2 \pi}} \int_{z}^{\infty} e^{-\frac{1}{2} t^{2}} \mathrm{~d} t,
$$

with the property $P(z)+Q(z)=1$.

In our analysis we prefer the notation in terms of the error function and complementary error function, which are defined by

$$
\operatorname{erf} z=\frac{2}{\sqrt{\pi}} \int_{0}^{z} e^{-t^{2}} \mathrm{~d} t, \quad \operatorname{erfc} z=\frac{2}{\sqrt{\pi}} \int_{z}^{\infty} e^{-t^{2}} \mathrm{~d} t,
$$

with the symmetry relations $\operatorname{erf} z+\operatorname{erfc} z=1$ and $\operatorname{erfc} z+\operatorname{erfc}(-z)=2$. These are related to the normal distribution by

$$
P(z)=\frac{1}{2} \operatorname{erfc}(-z / \sqrt{2}), \quad Q(z)=\frac{1}{2} \operatorname{erfc}(z / \sqrt{2}) .
$$

The uniform expansion of the incomplete gamma functions

In (Temme 1996, pp. 283-286) we derived the uniform expansion by using saddle point methods for integrals. In that analysis the complementary error function appeared because a singularity (a pole) of the integrand approaches the saddle point, when $a \sim z$. For details on the role of the complementary error function in such situations, see (Wong 2001, pp. 356-358).

We summarize the results by giving the following representations

$$
\begin{aligned}
& Q(a, z)=\frac{1}{2} \operatorname{erfc}(\eta \sqrt{a / 2})+R_{a}(\eta), \\
& P(a, z)=\frac{1}{2} \operatorname{erfc}(-\eta \sqrt{a / 2})-R_{a}(\eta),
\end{aligned}
$$

where

$$
\frac{1}{2} \eta^{2}=\lambda-1-\ln \lambda, \quad \lambda=\frac{z}{a},
$$

and

$$
R_{a}(\eta)=\frac{e^{-\frac{1}{2} a \eta^{2}}}{\sqrt{2 \pi a}} S_{a}(\eta), \quad S_{a}(\eta) \sim \sum_{n=0}^{\infty} \frac{C_{n}(\eta)}{a^{n}}
$$

as $a \rightarrow \infty$.

The relation between $\eta$ and $\lambda$ in (5.15) becomes clear when we expand

$$
\lambda-1-\ln \lambda=\frac{1}{2}(\lambda-1)^{2}-\frac{1}{3}(\lambda-1)^{3}+\frac{1}{4}(\lambda-1)^{4}+\cdots,
$$

and in fact the relation in (5.15) can also be written as

$$
\eta=(\lambda-1) \sqrt{\frac{2(\lambda-1-\ln \lambda)}{(\lambda-1)^{2}}},
$$

where the sign of the square root is positive for $\lambda>0$. For complex values 
we use analytic continuation. An expansion for small values of $|\lambda-1|$ reads

$$
\eta=(\lambda-1)-\frac{1}{3}(\lambda-1)^{2}+\frac{7}{36}(\lambda-1)^{3}+\cdots,
$$

and, upon inverting this expansion,

$$
\lambda=1+\eta+\frac{1}{3} \eta^{2}+\frac{1}{36} \eta^{3}+\cdots .
$$

Note that the symmetry relation $P(a, z)+Q(a, z)=1$ is preserved in the representations in (5.9) because $\operatorname{erfc} z+\operatorname{erfc}(-z)=2$.

We give the steps on determining the coefficients $C_{n}(\eta)$ in (5.16). Differentiating the relations in (5.14) of $Q(a, z)$ with respect to $\eta$ gives, on the one hand,

$$
\frac{\mathrm{d} Q(a, z)}{\mathrm{d} \eta}=\frac{\mathrm{d} Q(a, z)}{\mathrm{d} z} \frac{\mathrm{d} z}{\mathrm{~d} \eta}=-\frac{1}{\Gamma(a)} z^{a-1} e^{-z} \frac{\mathrm{d} z}{\mathrm{~d} \eta},
$$

and on the other hand, by using (5.14) and (5.16),

$$
\frac{\mathrm{d} Q(a, z)}{\mathrm{d} \eta}=\left[-\sqrt{\frac{a}{2 \pi}} \frac{\lambda-1}{\lambda}-\eta \sqrt{\frac{a}{2 \pi}} S_{a}(\eta)+\frac{1}{\sqrt{2 \pi a}} \frac{\mathrm{d} S_{a}(\eta)}{\mathrm{d} \eta}\right] e^{-\frac{1}{2} a \eta^{2}},
$$

where we have used

$$
\frac{\mathrm{d} z}{\mathrm{~d} \eta}=a \frac{\mathrm{d} \lambda}{\mathrm{d} \eta}=a \frac{\lambda \eta}{\lambda-1} .
$$

After straightforward manipulations we obtain

$$
\frac{\mathrm{d} S_{a}(\eta)}{\mathrm{d} \eta}-a \eta S_{a}(\eta)=a\left[1-\frac{\eta}{(\lambda-1) \Gamma^{*}(a)}\right],
$$

where $\Gamma^{*}(a)$ is defined by

$$
\Gamma^{*}(a)=\sqrt{\frac{a}{2 \pi}} e^{a} a^{-a} \Gamma(a), \quad a>0 .
$$

We have the expansion

$$
\frac{1}{\Gamma^{*}(a)} \sim \sum_{n=0}^{\infty} \gamma_{n} a^{-n}, \quad a \rightarrow \infty
$$

where the first few $\gamma_{n}$ are

$$
\gamma_{0}=1, \gamma_{1}=-\frac{1}{12}, \gamma_{2}=\frac{1}{288}, \gamma_{3}=\frac{139}{51840} .
$$

The numbers $\gamma_{n}$ also appear in the well-known asymptotic expansion of the Euler gamma function. That is,

$$
\Gamma^{*}(a) \sim \sum_{n=0}^{\infty}(-1)^{n} \gamma_{n} a^{-n}, \quad a \rightarrow \infty .
$$

We substitute the expansion of $S_{a}(\eta)$ given in (5.16) and the expansion 


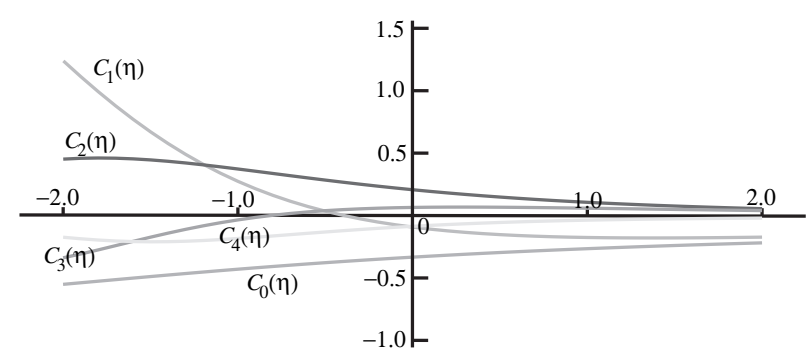

Figure 5.5. Graphs of the first 5 coefficients $C_{n}(\eta)$. Because of scaling we have drawn graphs of $\rho C_{n}(\eta)$, where $\rho=1,50,50,100,100$ for $n=0,1,2,3,4$, respectively.

(5.26) into the differential equation (5.24). Comparing, after these substitutions, equal powers of $a$ in (5.24), we obtain

$$
C_{0}(\eta)=\frac{1}{\lambda-1}-\frac{1}{\eta}
$$

and the recurrence relation

$$
\eta C_{n}(\eta)=\frac{d}{\mathrm{~d} \eta} C_{n-1}(\eta)+\frac{\eta}{\lambda-1} \gamma_{n}, \quad n \geq 1 .
$$

For $C_{1}(\eta)$ we have

$$
C_{1}(\eta)=\frac{1}{\eta^{3}}-\frac{1}{(\lambda-1)^{3}}-\frac{1}{(\lambda-1)^{2}}-\frac{1}{12(\lambda-1)} .
$$

The first two coefficients (and all higher coefficients) have a removable singularity at $\eta=0$, that is, at $\lambda=1$ or $z=a$. All $C_{n}(\eta)$ are analytic at the origin $\eta=0$.

The expansion in (5.16) has no restrictions on the parameter $\lambda$. It holds uniformly with respect to $\lambda \geq 0$ (and for complex values of $a$ and $\lambda$ ). So it is much more powerful than, for example, the asymptotic expansion (5.3). However, the computation of the coefficients $C_{n}(\eta)$ is not as easy as that of the coefficients in (5.3). In particular, near the transition point, that is, when $z \sim a$, the removable singularities in the representations of $C_{0}$ and $C_{1}$ as shown in (5.29) and (5.31) are difficult to handle in numerical computations. All higher coefficients show this type of cancellations, and the removable singularities in $C_{n}$ are poles of order $2 n+1$.

In Figure 5.5 we show the graphs of $C_{n}(\eta)$ for $n=0,1,2,3,4$, properly scaled in order to get them visible in one figure.

Expansions for the coefficients

We concentrate on the numerical aspects of the expansion of $S_{a}(\eta)$ in (5.16). We have already observed that the coefficients $C_{k}(\eta)$ in $(5.16)$ are difficult 
to evaluate near the transition point $\eta=0$, which corresponds to $\lambda=1$, or $z=a$. We will give two methods for evaluating the coefficients and the expansion.

The coefficients $C_{k}(\eta)$ are analytic (Temme 1996, pp. 283-286) inside the disk $|\eta|<2 \sqrt{\pi}=3.54 \ldots$. So, we can expand all coefficients in power series for $|\eta|<2 \sqrt{\pi}$. For numerical applications these expansions can be used for complex $\eta$, say, for $|\eta| \leq 1$. More efficiently, when the variables $a$ and $z$ are real and positive, we can expand the coefficients in terms of Chebyshev polynomials in intervals of the real $\eta$-axis.

We give the first terms in the Maclaurin expansions of the first coefficients:

$$
\begin{aligned}
& C_{0}(\eta)=-\frac{1}{3}+\frac{1}{12} \eta-\frac{2}{135} \eta^{2}+\frac{1}{864} \eta^{3}+\frac{1}{2835} \eta^{4}-\frac{139}{777600} \eta^{5}+\cdots, \\
& C_{1}(\eta)=-\frac{1}{540}-\frac{1}{288} \eta+\frac{1}{378} \eta^{2}-\frac{77}{77760} \eta^{3}+\frac{1}{4860} \eta^{4}-\frac{1}{2488320} \eta^{5}+\cdots, \\
& C_{2}(\eta)=\frac{25}{6048}-\frac{139}{51840} \eta+\frac{1}{1296} \eta^{2}+\frac{1}{497664} \eta^{3}-\frac{6199}{57736800} \eta^{4}+\cdots, \\
& C_{3}(\eta)=\frac{101}{155520}+\frac{571}{2488320} \eta-\frac{54179}{115473600} \eta^{2}+\frac{41969}{156764160} \eta^{3}-\frac{20639}{272937600} \eta^{4}+\cdots, \\
& C_{4}(\eta)=-\frac{3184811}{3695155200}+\frac{163879}{209018880} \eta-\frac{8707}{29113344} \eta^{2}-\frac{47207}{32248627200} \eta^{3}+\cdots
\end{aligned}
$$

In Section 5.3 we discuss alternative uniform expansions in which no coefficients occur that are difficult to compute. But first we give another numerical scheme for the expansion in (5.16).

Numerical algorithm for small values of $\eta$

Instead of expanding each coefficient $C_{n}(\eta)$ in powers of $\eta$, which needs the storage of many coefficients, we expand the function $S_{a}(\eta)$ of $(5.16)$ in powers of $\eta$. The coefficients are functions of $a$, and we write

$$
S_{a}(\eta)=\sum_{n=0}^{\infty} \alpha_{n} \eta^{n},
$$

where the series again converges for $|\eta|<2 \sqrt{\pi}$.

To compute the coefficients $\alpha_{n}$, we use the differential equation for $S_{a}(\eta)$ given in (5.24). Substituting the expansion (5.33) into (5.24), using the coefficients $d_{n}$ in the expansion

$$
\frac{\eta}{\lambda-1}=\sum_{n=0}^{\infty} d_{n} \eta^{n},
$$

we obtain for $\alpha_{n}$ the recurrence relation

$$
\alpha_{n}=\frac{1}{a}(n+2) \alpha_{n+2}+\frac{d_{n+1}}{\Gamma^{*}(a)}, \quad n=0,1,2, \ldots .
$$


The coefficients $d_{n}$ follow from the coefficients of $C_{0}(\eta)$, of which the first few are given in (5.32), because $\eta /(\lambda-1)=1+\eta C_{0}(\eta)$. We have

$$
d_{0}=1, \quad d_{1}=-\frac{1}{3}, \quad d_{2}=-\frac{1}{12}, \quad d_{3}=-\frac{2}{135}, \quad d_{4}=\frac{1}{864}, \quad d_{5}=\frac{1}{2835} .
$$

With these values we can compute the first terms with odd index:

$$
\begin{aligned}
\alpha_{1} & =\frac{a}{\Gamma^{*}(a)}\left[\Gamma^{*}(a)-1\right], \\
\alpha_{3} & =\frac{a^{2}}{1 \cdot 3 \Gamma^{*}(a)}\left[\Gamma^{*}(a)-1-\frac{1}{12 a}\right], \\
\alpha_{5} & =\frac{a^{2}}{1 \cdot 3 \cdot 5 \Gamma^{*}(a)}\left[\Gamma^{*}(a)-1-\frac{1}{12 a}-\frac{1}{288 a^{2}}\right] .
\end{aligned}
$$

We observe, see (5.27) and (5.28), that the computation of these $\alpha_{n}$ requires not only the value of $\Gamma^{*}(a)$, but also that of $\Gamma^{*}(a)$ with the first terms of the asymptotic expansion subtracted. The higher odd coefficients show the same pattern; more and more terms of the asymptotic expansions have to be subtracted. In fact we recur remainders of the asymptotic expansion of the gamma function. In particular, when $a$ is large, this is a very unstable process. The same problems arise with the even coefficients. Note that $\alpha_{0}=S_{a}(0)$, a quantity that can be computed from an asymptotic expansion, and the higher even terms follow from the recursion in (5.35), with more and more terms subtracted in this expansion of $S_{a}(0)$.

When we use (5.35) in the backward direction the recursion becomes stable. In addition, we do not need the computation of $S_{a}(0)$ and $\Gamma^{*}(a)$, because these values follow from the backward recursion process. We only need enough coefficients $d_{n}$ of (5.34) for this recursion.

First we remove $\Gamma^{*}(a)$ from the recursion in (5.35) by writing

$$
\alpha_{n}=\frac{\beta_{n}}{\Gamma^{*}(a)}, \quad n=0,1,2, \ldots,
$$

which gives for $\beta_{n}$ the recursion

$$
\beta_{n}=\frac{1}{a}(n+2) \beta_{n+2}+d_{n+1}, \quad n=0,1,2, \ldots .
$$

We choose a positive integer $N$, put $\beta_{N+2}=\beta_{N+1}=0$ and compute the sequence

$$
\beta_{N}, \beta_{N-1}, \ldots, \beta_{1}, \beta_{0}
$$

from the recurrence relation (5.39). Because

$$
\Gamma^{*}(a)=1+\frac{1}{a} \beta_{1}
$$




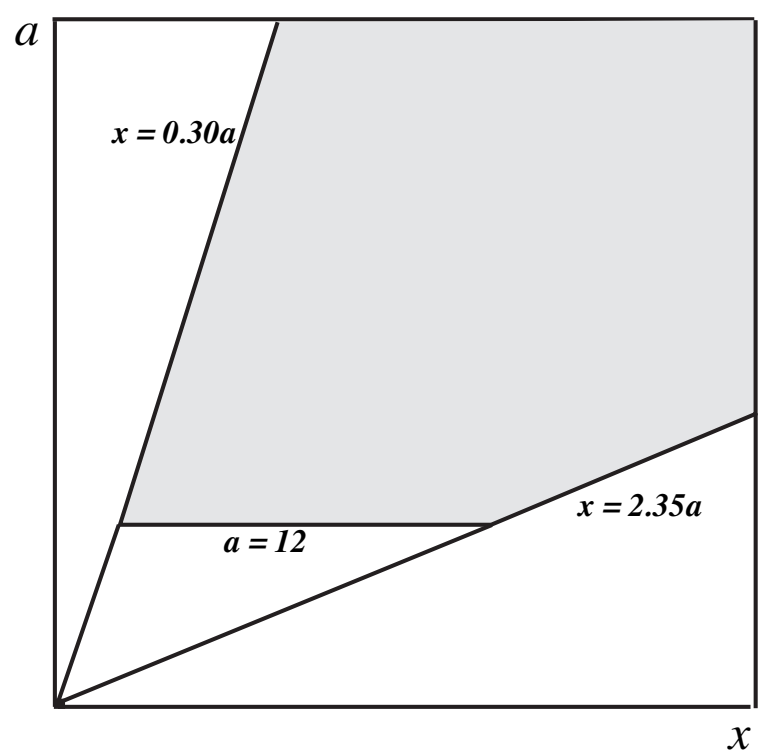

Figure 5.6. The domain of application (grey) where we can apply the backward recursion scheme (5.39) to obtain IEEE double precision values of $S_{a}(\eta)$. The grey domain is bounded by the lines $a=12, x=2.35 a$ and $x=0.30 a$.

we have

$$
S_{a}(\eta) \sim \frac{a}{a+\beta_{1}} \sum_{n=0}^{N} \beta_{n} \eta^{n}
$$

as an approximation for $S_{a}(\eta)$.

We verify this algorithm by taking several values of $a$ and $N=25$ and $N=35$. In Table 5.5 we give the relative errors of the approximations of $\Gamma(a)$, which are computed by using $\beta_{1}$ in (5.41), and by computing $\Gamma(a)$ from the relation in (5.25). For example, with $N=25$ and $a=5$ we obtain

$$
\Gamma(a)=23.999999999892 \ldots, \quad \text { with relative error } 0.45_{10}(-11) \text {. }
$$

We observe that for the larger values of $a$ the scheme gives better approximations, as is the case for the larger value of $N$.

We have used the approximation in (5.42) for computing the incomplete gamma functions in IEEE double precision for $a \geq 12$ and $|\eta| \leq 1$. We need the storage of 25 coefficients $d_{n}$, and in the series in (5.42) we need 25 terms or less.

The value $\eta=-1$ corresponds to $\lambda=0.30 \ldots$, and the value $\eta=1$ with $\lambda=2.35 \ldots$ In Figure 5.6 we show the area in the $(x, a)$ quarter plane 
Table 5.5. Relative errors $\delta$ in the computation of $\Gamma(a)$ by using the backward recursion scheme (5.39) for several values of $a$ and $N=25$ and $N=35$.

\begin{tabular}{|c|c|c|c|c|c|c|}
\hline$N$ & $a$ & $\delta$ & $a$ & $\delta$ & $a$ & $\delta$ \\
\hline$N=25$ & $\left|\begin{array}{l}2 \\
3 \\
4 \\
5 \\
6 \\
7\end{array}\right|$ & $\begin{array}{l}0.39_{10}(-06) \\
0.27_{10}(-08) \\
0.75_{10}(-10) \\
0.45_{10}(-11) \\
0.44_{10}(-12) \\
0.61_{10}(-13)\end{array}$ & $\begin{array}{r}8 \\
9 \\
10 \\
11 \\
12 \\
13\end{array}$ & $\begin{array}{l}0.11_{10}(-13) \\
0.24_{10}(-14) \\
0.61_{10}(-15) \\
0.18_{10}(-15) \\
0.58_{10}(-16) \\
0.21_{10}(-16)\end{array}$ & $\begin{array}{l}14 \\
15 \\
16 \\
17 \\
18 \\
19\end{array}$ & $\begin{array}{l}0.79_{10}(-17) \\
0.32_{10}(-17) \\
0.14_{10}(-17) \\
0.64_{10}(-18) \\
0.30_{10}(-18) \\
0.15_{10}(-18)\end{array}$ \\
\hline$N=35$ & \begin{tabular}{|l}
2 \\
3 \\
4 \\
5 \\
6 \\
7
\end{tabular} & $\begin{array}{l}0.80_{10}(-06) \\
0.57_{10}(-09) \\
0.30_{10}(-11) \\
0.50_{10}(-13) \\
0.17_{10}(-14) \\
0.93_{10}(-16)\end{array}$ & $\begin{array}{r}8 \\
9 \\
10 \\
11 \\
12 \\
13\end{array}$ & $\begin{array}{l}0.76_{10}(-17) \\
0.82_{10}(-18) \\
0.11_{10}(-18) \\
0.18_{10}(-19) \\
0.35_{10}(-20) \\
0.77_{10}(-21)\end{array}$ & $\begin{array}{l}14 \\
15 \\
16 \\
17 \\
18 \\
19\end{array}$ & $\begin{array}{l}0.19_{10}(-21) \\
0.50_{10}(-22) \\
0.15_{10}(-22) \\
0.46_{10}(-23) \\
0.15_{10}(-23) \\
0.54_{10}(-24)\end{array}$ \\
\hline
\end{tabular}

where we can apply the algorithm to obtain double precision. The domain is bounded by the lines $a=12, x=2.35 a$ and $x=0.30 a$.

\subsection{A simpler uniform expansion}

The expansion considered in Section 5.2 can be modified to obtain an expansion with coefficients that can be evaluated much more easily than the coefficients $C_{n}(\eta)$. The modified expansion is again valid for large values of $a$, it is again valid in the transition area $a \sim z$. The restriction on $\lambda=z / a$, however, is $\lambda-1=o\left(a^{-\frac{1}{3}}\right)$ as $a \rightarrow \infty$.

We start with the integral (see (5.1))

$$
\Gamma(a+1, z)=\int_{z}^{\infty} t^{a} e^{-t} \mathrm{~d} t
$$

and we consider positive parameters $a$ and $z$. We substitute $t=a(1+s)$. This gives

$$
\Gamma(a+1, z)=a^{a+1} e^{-a} \int_{\mu}^{\infty} e^{-a[s-\ln (1+s)]} \mathrm{d} s, \quad \mu=\lambda-1=\frac{z-a}{a} .
$$

The exponential function has a maximum at the origin $s=0$. We write

$$
\Gamma(a+1, z)=a^{a+1} e^{-a} \int_{\mu}^{\infty} e^{-\frac{1}{2} a s^{2}-a f(s)} \mathrm{d} s,
$$


where

$$
f(s)=s-\frac{1}{2} s^{2}-\ln (1+s)=\mathcal{O}\left(s^{3}\right), \quad s \rightarrow 0 .
$$

We expand

$$
e^{-a f(s)}=\sum_{n=0}^{\infty} D_{n}(a) s^{n}, \quad|s|<1,
$$

and upon substituting this expansion in (5.46), we obtain the expansion

$$
\Gamma(a+1, z) \sim a^{a+1} e^{-a} \sum_{n=0}^{\infty} D_{n}(a) \Phi_{n}(a, z),
$$

where

$$
\Phi_{n}(a, z)=\int_{\mu}^{\infty} s^{n} e^{-\frac{1}{2} a s^{2}} \mathrm{~d} s, \quad n=0,1,2, \ldots .
$$

The first two $\Phi_{n}$ are

$$
\Phi_{0}(a, z)=\sqrt{\frac{\pi}{2 a}} \operatorname{erfc}(\mu \sqrt{a / 2}), \quad \Phi_{1}(a, z)=\frac{1}{a} e^{-\frac{1}{2} a \mu^{2}} .
$$

By integrating by parts in (5.50) it easily follows that

$$
a \Phi_{n+1}(a, z)=n \Phi_{n-1}(a, z)+\mu^{n} e^{-\frac{1}{2} a \mu^{2}}, \quad n=1,2,3, \ldots .
$$

We can also derive a recurrence relation for the coefficients. We have the starting values $D_{0}(a)=1, D_{1}(a)=0$, and by differentiating (5.48), we obtain

$$
(n+1) D_{n+1}(a)=a D_{n-2}(a)-n D_{n}(a), \quad n=1,2,3, \ldots .
$$

The expansion in (5.50) is of interest because the coefficients $D_{n}(a)$ can be computed very easily by using the recursion in (5.53). Further, the recursion for the $\Phi_{n}$ is quite simple.

The expansion, with proofs for complex values of $a$ and $z$, is derived in (Ferreira, López and Pérez Sinusía 2005). In this reference several other expansions of the incomplete gamma function are considered. See also (Paris 2002).

We observe that a similar expansion can be derived for the other incomplete gamma function $\gamma(a+1, z)$. In that case the functions $\Phi_{n}$ should be replaced by functions $\Psi_{n}$ defined by

$$
\Psi_{n}(a, z)=\int_{-1}^{\mu} s^{n} e^{-\frac{1}{2} a s^{2}} \mathrm{~d} s, \quad n=0,1,2, \ldots .
$$

The function $\Psi_{0}$ can be expressed in terms of two error functions, and the other ones follow from integrating by parts.

From a numerical example we conclude that the asymptotic convergence 
of the expansion (5.49) is quite slow. When we take $z=100$ and $a=101$, and we sum the series with 12 terms, we obtain 4 significant digits. This result does not improve when we take more terms.

\subsection{Airy-type expansions for Bessel functions}

Airy functions are solutions of the differential equation

$$
w^{\prime \prime}-z w=0 .
$$

Two linearly independent solutions that are real for real values of $z$ are denoted by $\operatorname{Ai}(z)$ and $\operatorname{Bi}(z)$. Equation (5.55) is the simplest second-order linear differential equation that has a simple turning point (at $z=0$ ). More general turning point equations have the standard form

$$
\frac{\mathrm{d}^{2} W}{\mathrm{~d} \zeta^{2}}=\left[u^{2} \zeta+\psi(\zeta)\right] W,
$$

and the problem is to find an asymptotic approximation of $W(\zeta)$ for large values of $u$, that holds uniformly in a neighbourhood of $\zeta=0$. A first approximation is obtained by neglecting $\psi(\zeta)$, which gives the solutions

$$
\operatorname{Ai}\left(u^{\frac{2}{3}} \zeta\right), \quad \operatorname{Bi}\left(u^{\frac{2}{3}} \zeta\right) .
$$

For a detailed discussion of this kind of problem we refer to (Olver 1997, Chapter 11). Many solutions of physical problems and many special functions can be transformed into the standard form (5.56). Examples are Bessel functions, Whittaker functions, the classical orthogonal polynomials (in particular Hermite and Laguerre polynomials), and parabolic cylinder functions.

In all known cases the coefficients are difficult to compute in the neighbourhood of the turning point, and we saw a similar difficulty in the uniform expansion of the incomplete gamma functions in Section 5.2.

In this section we discuss two methods for computing the asymptotic series. One method is based on expanding the coefficients in the series into Maclaurin series. In the second method we consider the computation of auxiliary functions that can be computed more efficiently than the coefficients in the first method, and we do not need the tabulation of many coefficients. This method is similar to that of described for the computation of incomplete gamma functions in Section 5.2.

The methods described in this section are quite general, but we only treat the case of Bessel functions, by using the differential equation of the Bessel functions, which has a turning point character when the order and argument of the Bessel functions are equal. 
The Airy-type asymptotic expansions

The ordinary Bessel functions $J_{\nu}(z)$ and $Y_{\nu}(z)$, and all other Bessel functions, can be expanded in terms of Airy functions. We give the transformations of the Bessel differential equation

$$
z^{2} f^{\prime \prime}+z f^{\prime}+\left(z^{2}-\nu^{2}\right) f=0
$$

into the form (5.56). First we change the variable $z$ into $\nu z$ and apply a transformation to remove the first derivative term. We obtain the equation

$$
F^{\prime \prime}+\left(\nu^{2} \frac{z^{2}-1}{z^{2}}+\frac{1}{4 z^{2}}\right) F=0,
$$

with solutions $\sqrt{z} J_{\nu}(\nu z)$ and $\sqrt{z} Y_{\nu}(\nu z)$. The turning point character at $z=1$ of this equation is visible now, and we transform this point to the origin by using the transformation

$$
\zeta\left(\frac{\mathrm{d} \zeta}{\mathrm{d} z}\right)^{2}=\frac{1-z^{2}}{z^{2}}, \quad W=\sqrt{\zeta^{\prime}} w
$$

This transformation gives the equation (5.56) with $\psi(\zeta)$ given by

$$
\psi(\zeta)=\frac{5}{16 \zeta^{2}}+\frac{\zeta z^{2}\left(z^{2}+4\right)}{4\left(z^{2}-1\right)^{3}}
$$

and solutions

$$
\sqrt{z} \sqrt{\zeta^{\prime}} J_{\nu}(\nu z), \quad \sqrt{z} \sqrt{\zeta^{\prime}} Y_{\nu}(\nu z)
$$

The transformations used here are Liouville transformations; see also (Olver 1997, p. 398).

Because the Airy functions given in (5.57) are solutions of (5.56) when $\psi(\zeta)$ is neglected, and because of asymptotic properties of the Bessel functions, the following representations are chosen:

$$
\begin{aligned}
& J_{\nu}(\nu z)=\frac{\phi(\zeta)}{\nu^{\frac{1}{3}}}\left[\operatorname{Ai}\left(\nu^{\frac{2}{3}} \zeta\right) A_{\nu}(\zeta)+\nu^{-\frac{4}{3}} \operatorname{Ai}^{\prime}\left(\nu^{\frac{2}{3}} \zeta\right) B_{\nu}(\zeta)\right], \\
& Y_{\nu}(\nu z)=-\frac{\phi(\zeta)}{\nu^{\frac{1}{3}}}\left[\operatorname{Bi}\left(\nu^{\frac{2}{3}} \zeta\right) A_{\nu}(\zeta)+\nu^{-\frac{4}{3}} \operatorname{Bi}^{\prime}\left(\nu^{\frac{2}{3}} \zeta\right) B_{\nu}(\zeta)\right],
\end{aligned}
$$

where

$$
\phi(\zeta)=\left(\frac{4 \zeta}{1-z^{2}}\right)^{\frac{1}{4}}, \quad \phi(0)=2^{\frac{1}{3}} .
$$

The new variable $\zeta$ introduced in (5.60) can be written as

$$
\begin{aligned}
& \frac{2}{3} \zeta^{3 / 2}=\ln \frac{1+\sqrt{1-z^{2}}}{z}-\sqrt{1-z^{2}}, \quad 0 \leq z \leq 1, \\
& \frac{2}{3}(-\zeta)^{3 / 2}=\sqrt{z^{2}-1}-\arccos \frac{1}{z}, \quad z \geq 1 \text {. }
\end{aligned}
$$


Next we introduce asymptotic expansions for the functions $A_{\nu}(\zeta)$ and $B_{\nu}(\zeta)$ of (5.63). It appears, after substituting the Bessel functions in (5.63) into (5.56) that we have the formal expansions

$$
A_{\nu}(\zeta) \sim \sum_{s=0}^{\infty} \frac{a_{s}(\zeta)}{\nu^{2 s}}, \quad B_{\nu}(\zeta) \sim \sum_{s=0}^{\infty} \frac{b_{s}(\zeta)}{\nu^{2 s}}
$$

The first coefficients

$$
a_{0}(\zeta)=1, \quad b_{0}(\zeta)=-\frac{5}{48 \zeta^{2}}+\frac{\phi^{2}(\zeta)}{48 \zeta}\left[\frac{5}{1-z^{2}}-3\right],
$$

where $\phi(\zeta)$ is given by (5.64). Higher coefficients follow certain recurrence relations. Further details on the coefficients are given later.

For the derivatives we have

$$
\begin{aligned}
& J_{\nu}^{\prime}(\nu z)=-\widehat{\phi}(\zeta)\left[\nu^{-\frac{4}{3}} \operatorname{Ai}\left(\nu^{\frac{2}{3}} \zeta\right) C_{\nu}(\zeta)+\nu^{-\frac{2}{3}} \operatorname{Ai}^{\prime}\left(\nu^{\frac{2}{3}} \zeta\right) D_{\nu}(\zeta)\right], \\
& Y_{\nu}^{\prime}(\nu z)=\widehat{\phi}(\zeta)\left[\nu^{-\frac{4}{3}} \operatorname{Bi}\left(\nu^{\frac{2}{3}} \zeta\right) C_{\nu}(\zeta)+\nu^{-\frac{2}{3}} \operatorname{Bi}^{\prime}\left(\nu^{\frac{2}{3}} \zeta\right) D_{\nu}(\zeta)\right],
\end{aligned}
$$

where

$$
\widehat{\phi}(\zeta)=-\frac{\mathrm{d} \zeta}{\mathrm{d} z} \phi(\zeta)=\frac{2}{z \phi(\zeta)}, \quad \chi(\zeta)=\frac{\phi^{\prime}(\zeta)}{\phi(\zeta)}=\frac{4-z^{2}[\phi(\zeta)]^{6}}{16 \zeta}
$$

and

$$
\begin{aligned}
C_{\nu}(\zeta) & =\chi(\zeta) A_{\nu}(\zeta)+A_{\nu}^{\prime}(\zeta)+\zeta B_{\nu}(\zeta), \\
D_{\nu}(\zeta) & =A_{\nu}(\zeta)+\nu^{-2} \chi(\zeta) B_{\nu}(\zeta)+\nu^{-2} B_{\nu}^{\prime}(\zeta) .
\end{aligned}
$$

Primes denote differentiation with respect to $\zeta$.

The functions $C_{\nu}(\zeta), D_{\nu}(z)$ have the expansions

$$
C_{\nu}(\zeta) \sim \sum_{s=0}^{\infty} \frac{c_{s}(\zeta)}{\nu^{2 s}}, \quad D_{\nu}(\zeta) \sim \sum_{s=0}^{\infty} \frac{d_{s}(\zeta)}{\nu^{2 s}}
$$

where

$$
\begin{aligned}
& c_{s}(\zeta)=\chi(\zeta) a_{s}(\zeta)+a_{s}^{\prime}(\zeta)+\zeta b_{s}(\zeta), \\
& d_{s}(\zeta)=a_{s}(\zeta)+\chi(\zeta) b_{s-1}(\zeta)+b_{s-1}^{\prime}(\zeta) .
\end{aligned}
$$

The Airy-type asymptotic expansions of this section hold as $\nu \rightarrow \infty$, uniformly with respect to $z \in[0, \infty)$ and for complex values of $\nu$ and $z$ (Olver 1997, Chapter 11) .

The first coefficients $c_{s}, d_{s}$ of (5.71) are

$$
c_{0}(\zeta)=\frac{7}{48 \zeta}+\frac{\phi^{2}(\zeta)}{48}\left[9-\frac{7}{1-z^{2}}\right], \quad d_{0}(\zeta)=1 .
$$


A recursive scheme for evaluating $a_{s}, b_{s}$ is given by

$$
\begin{array}{ll}
a_{s}^{\prime \prime}(\zeta)+2 \zeta b_{s}^{\prime}(\zeta)+b_{s}(\zeta)-\psi(\zeta) a_{s}(\zeta) & =0 \\
2 a_{s+1}^{\prime}(\zeta)+b_{s}^{\prime \prime}(\zeta)-\psi(\zeta) b_{s}(\zeta) & =0
\end{array}
$$

where $a_{0}(\zeta)=1$ and $\psi(\zeta)$ is given by $(5.61)$.

Properties of the functions $A_{\nu}, B_{\nu}, C_{\nu}, D_{\nu}$

By using equation (5.56), with $\psi(\zeta)$ as given in (5.61), it is straightforward to derive the following system of differential equations for the functions $A_{\nu}(\zeta), B_{\nu}(\zeta)$

$$
\begin{aligned}
& A^{\prime \prime}+2 \zeta B^{\prime}+B-\psi(\zeta) A=0 \\
& B^{\prime \prime}+2 \nu^{2} A^{\prime}-\psi(\zeta) B=0
\end{aligned}
$$

where primes denote differentiation with respect to $\zeta$.

A Wronskian for the system (5.75) follows by eliminating the terms with $\psi(\zeta)$. This gives

$$
A^{\prime \prime} B-B^{\prime \prime} A+B^{2}+2 \zeta B^{\prime} B-2 \nu^{2} A^{\prime} A=0,
$$

which can be integrated as follows

$$
\nu^{2} A_{\nu}^{2}(\zeta)+A_{\nu}(\zeta) B_{\nu}^{\prime}(\zeta)-A_{\nu}^{\prime}(\zeta) B_{\nu}(\zeta)-\zeta B_{\nu}^{2}(\zeta)=\nu^{2} .
$$

The constant on the right-hand side follows by taking $\zeta=0$ and from information given later in this section.

Expansions for $a_{s}(\zeta), b_{s}(\zeta), c_{s}(\zeta), d_{s}(\zeta)$

The singular points of the functions $z(\zeta), \psi(\zeta), \phi(\zeta), \widehat{\phi}(\zeta), \chi(\zeta)$ and those of the coefficients of the asymptotic expansions occur at

$$
\zeta^{ \pm}=\left(\frac{3}{2} \pi\right)^{\frac{2}{3}} e^{ \pm i \pi / 3}
$$

These points correspond to $z=e^{\mp \pi \mathrm{i}}$. It follows that the radius of convergence of the Maclaurin series of these quantities equals $2.81 \cdots$. In this section we give the expansions and mention the values of the early coefficients.

It is convenient to start with an expansion of $z$ in powers of $\zeta$. We substitute $z=1+z_{1} \zeta+\cdots$ into the first part of (5.60). This gives $z_{1}^{3}=-1 / 2$. Using the relations in (5.65) we obtain the correct branch: $z_{1}=-2^{-\frac{1}{3}}$. We write

$$
\zeta=2^{\frac{1}{3}} \eta
$$


and we obtain in a straightforward way the following expansions:

$$
\begin{aligned}
& z(\zeta)=\quad \sum_{n=0}^{\infty} z_{n} \eta^{n}=\quad\left[1-\eta+\frac{3}{10} \eta^{2}+\frac{1}{350} \eta^{3}-\frac{479}{63000} \eta^{4}+\cdots\right] \\
& \psi(\zeta)=2^{\frac{1}{3}} \sum_{n=0}^{\infty} \psi_{n} \eta^{n}=2^{\frac{1}{3}}\left[\frac{1}{70}+\frac{2}{75} \eta+\frac{138}{13475} \eta^{2}-\frac{296}{73125} \eta^{3}-\frac{38464}{7074375} \eta^{4}+\cdots\right] \\
& \phi(\zeta)=2^{\frac{1}{3}} \sum_{n=0}^{\infty} \phi_{n} \eta^{n}=2^{\frac{1}{3}}\left[1+\frac{1}{5} \eta+\frac{9}{350} \eta^{2}-\frac{89}{15750} \eta^{3}-\frac{4547}{1155000} \eta^{4}+\cdots\right] \\
& \widehat{\phi}(\zeta)=2^{\frac{2}{3}} \sum_{n=0}^{\infty} \widehat{\phi}_{n} \eta^{n}=2^{\frac{2}{3}}\left[1+\frac{4}{5} \eta+\frac{18}{35} \eta^{2}+\frac{88}{315} \eta^{3}+\frac{79586}{606375} \eta^{4}+\cdots\right] \\
& \chi(\zeta)=2^{-\frac{1}{3}} \sum_{n=0}^{\infty} \chi_{n} \eta^{n}=2^{-\frac{1}{3}}\left[\frac{1}{5}+\frac{2}{175} \eta-\frac{64}{2625} \eta^{2}-\frac{30424}{3031875} \eta^{3}+\cdots\right]
\end{aligned}
$$

Next we consider the coefficients $a_{s}, b_{s}$ that are used in (5.66). We expand

$$
a_{s}(\zeta)=\sum_{t=0}^{\infty} a_{s}^{t} \eta^{t}, \quad b_{s}(\zeta)=2^{\frac{1}{3}} \sum_{t=0}^{\infty} b_{s}^{t} \eta^{t},
$$

where $\eta$ is given in (5.79). The coefficients $a_{s}^{t}, b_{s}^{t}$ are rational numbers. We know that $a_{0}(\zeta)=1$. Substituting the expansions in (5.74) we obtain recurrence relations for the coefficients $a_{s}^{t}, b_{s}^{t}$. We give the expansions of the first few terms:

$$
\begin{aligned}
& a_{0}(\zeta)=1 \\
& a_{1}(\zeta)=-\frac{1}{225}-\frac{71}{38500} \eta+\frac{82}{73125} \eta^{2}+\frac{5246}{3898125} \eta^{3}+\frac{185728}{478603125} \eta^{4}+\cdots, \\
& a_{2}(\zeta)=\frac{151439}{218295000}+\frac{68401}{147262500} \eta-\frac{1796498167}{4193689500000} \eta^{2}-\frac{583721053}{830718281250} \eta^{3}+\cdots \\
& a_{3}(\zeta)=-\frac{887278009}{2504935125000}-\frac{3032321618951}{9708942993750000} \eta+\cdots \\
& b_{0}(\zeta)=2^{\frac{1}{3}}\left[\frac{1}{70}+\frac{2}{225} \eta+\frac{138}{67375} \eta^{2}-\frac{296}{511875} \eta^{3}-\frac{38464}{63669375} \eta^{4}+\cdots\right] \\
& b_{1}(\zeta)=2^{\frac{1}{3}}\left[-\frac{1213}{1023750}-\frac{3757}{2695000} \eta-\frac{3225661}{6700443750} \eta^{2}+\frac{90454643}{336992906250} \eta^{3}+\cdots\right] \\
& b_{2}(\zeta)=2^{\frac{1}{3}}\left[\frac{16542537833}{37743205500000}+\frac{115773498223}{162820783125000} \eta+\frac{548511920915149}{1721719224225000000} \eta^{2}+\cdots\right], \\
& b_{3}(\zeta)=2^{\frac{1}{3}}\left[-\frac{9597171184603}{25476663712500000}-\frac{430990563936859253}{568167343994250000000} \eta+\cdots\right]
\end{aligned}
$$

Expansions for the coefficients $c_{s}, d_{s}$ are not really needed, because these quantities follow from the relations in (5.72), if expansions for the functions in the right-hand sides of (5.72) are available. 
Evaluation of the functions $A_{\nu}(\zeta), B_{\nu}(\zeta)$ by iteration

We now concentrate on solving the system of differential equations in (5.75) by using analytical techniques. Instead of expanding the coefficients $a_{s}, b_{s}$ of the asymptotic series we expand the functions $A_{\nu}(\zeta), B_{\nu}(\zeta)$ in Maclaurin series. As remarked earlier, the singular points of these functions occur at $\zeta^{ \pm}=(3 \pi / 2)^{\frac{2}{3}} e^{ \pm \mathrm{i} \pi / 3}$, and the radius of convergence of the series of $A_{\nu}(\zeta)$ and $B_{\nu}(\zeta)$ in powers of $\zeta$ equals $2.81 \cdots$.

We expand

$$
A_{\nu}(\zeta)=\sum_{n=0}^{\infty} f_{n}(\nu) \zeta^{n}, \quad B_{\nu}(\zeta)=\sum_{n=0}^{\infty} g_{n}(\nu) \zeta^{n}, \quad \psi(\zeta)=\sum_{n=0}^{\infty} h_{n} \zeta^{n}
$$

The coefficients $f_{0}, f_{1}, \ldots, g_{0}, g_{1}, \ldots$ are to be determined, while the coefficients $h_{n}$ are known. The first few $h_{n}$ follow from (5.79) and the second line in (5.80):

$$
h_{0}=\frac{1}{70} 2^{\frac{1}{3}}, \quad h_{1}=\frac{2}{75}, \quad h_{2}=\frac{69}{13475} 2^{\frac{2}{3}}, \quad h_{3}=\frac{148}{73125} 2^{\frac{1}{3}} .
$$

Upon substituting the expansions into (5.75), we obtain for $n=0,1,2, \ldots$ the recurrence relations

$$
\begin{array}{ll}
(n+2)(n+1) f_{n+2}+(2 n+1) g_{n}=\rho_{n}, & \rho_{n}=\sum_{k=0}^{n} h_{k} f_{n-k}, \\
(n+2)(n+1) g_{n+2}+2 \nu^{2}(n+1) f_{n+1}=\sigma_{n}, & \sigma_{n}=\sum_{k=0}^{n} h_{k} g_{n-k} .
\end{array}
$$

The solution $\left\{f_{n}, g_{n}\right\}$ of the set of recursions (5.86) cannot be obtained by forward recursion, because of instabilities. The same problems arise as in the case of the incomplete gamma functions described in Section 5.2. We give an iteration scheme to solve the recursions in the backward direction. The scheme runs as follows; for details we refer to (Temme 1997).

We choose an appropriate pair of functions $F_{0}, G_{0}$, and define two sequences of functions $\left\{F_{m}\right\},\left\{G_{m}\right\}$ by writing for $m=1,2,3, \ldots$ :

$$
F_{m}^{\prime \prime}+2 \zeta G_{m}^{\prime}+G_{m}=\psi(\zeta) F_{m-1}, \quad G_{m}^{\prime \prime}+2 \nu^{2} F_{m}^{\prime}=\psi(\zeta) G_{m-1} .
$$

We rewrite (5.86) in backward form:

$$
\begin{aligned}
& f_{n}=\frac{1}{2 \nu^{2}}\left[\frac{1}{n} \sigma_{n-1}-(n+1) g_{n+1}\right], \\
& g_{n-1}=\frac{1}{2 n-1}\left[\rho_{n-1}-n(n+1) f_{n+1}\right],
\end{aligned}
$$

where $n \geq 1$. The coefficients are assumed to belong to the functions $F_{m}(\zeta), G_{m}(\zeta)$ of the iteration process described by (5.87), while the coefficients $\rho_{n-1}$ and $\sigma_{n-1}$ are assumed to be known, and contain Maclaurin 
coefficients of $F_{m-1}(\zeta), G_{m-1}(\zeta)$ and $\psi(\zeta)$. Observe that (5.88) does not define $f_{0}$. After having computed $f_{1}, f_{2}, \ldots$, and $g_{0}, g_{1}, g_{2}, \ldots$ by the backward recursion process, we compute $f_{0}$ from the Wronskian (5.77):

$$
f_{0}=\frac{-g_{1}+\sqrt{g_{1}^{2}+4 \nu^{2}\left(\nu^{2}+f_{1} g_{0}\right)}}{2 \nu^{2}}
$$

where the + sign of the square root is taken because of the known behaviour of $F_{\nu}(0)$ when $\nu$ is large.

\section{Verifying the iterative scheme by numerical experiments}

For numerical applications information is needed about the growth of the coefficients $f_{n}, g_{n}$. Since the Maclaurin series in (5.84) have a radius of convergence equal to $2.81 \cdots$, for all values of $\nu$, the size of the coefficients $f_{n}, g_{n}$ is comparable with that of $h_{n}$. The number of coefficients $f_{n}, g_{n}$ needed in (5.84) also depends on the size of $\zeta$. When $|\zeta|=1$ we need about 45 terms in the Maclaurin series in (5.84) in order to obtain an accuracy of about 20 decimal digits. The $\zeta$-interval $[-1,1]$ corresponds to the $z$-interval $[0.39,1.98]$. When $z$ is outside this interval many other efficient algorithms are available for the computation of $J_{\nu}(\nu z), Y_{\nu}(\nu z)$.

We have computed successive iterates of Maclaurin coefficients $f_{n}, g_{n}$ defined in (5.84) for different values of $\nu$.

During each iteration we start the backward recursions with $f_{n}=g_{n-1}=$ $0, n \geq 46$, and we compute $f_{45}, g_{44}, f_{44}, g_{43}, \ldots$ by using (5.88) and (5.89). We use $h_{k}, k=0,1, \ldots, 45$ and recompute the coefficients $\rho_{k}, \sigma_{k}, k=0,1,2, \ldots, 45$, using (5.86) with values $f_{k}, g_{k}$ obtained in the previous iteration. In Table 5.6 we show the relative errors in the values $f_{0}, g_{0}, f_{5}, g_{5}$, when compared with more accurate values $f_{0}^{a}$, etc. Computations are done with Digits $=$ 20 . The accurate values are obtained by applying the backward recursion by using 10 iterations. We also give the relative error in the Wronskian relation (5.77) at $\zeta=1$ during each iteration.

From Table 5.6 we conclude that for $\nu=5$ we can already obtain an accuracy of $10^{-10}$ in the Wronskian after two iterations; further iterations improve the results. For larger values of $\nu$ the algorithm is very efficient.

\subsection{Asymptotic expansions: Concluding remarks}

The methods of this section can be used for Airy-type asymptotic expansions for other special functions. We mention as interesting cases parabolic cylinder functions, Coulomb wave functions, and other members of the class of Whittaker functions. To stay in the class of Bessel functions, we mention the modified Bessel function of the third kind $K_{i \nu}(z)$ of imaginary order, which plays an important role in the diffraction theory of pulses and in the study of certain hydrodynamical studies. Moreover, this function is the ker- 
Table 5.6. Relative errors during five iterations $(i)$ of $f_{0}, g_{0}, f_{5}, g_{5}$ compared with more accurate values $f_{0}^{a}$, etc. The final column shows the relative error in the Wronskian (5.77) at $\zeta=1$.

\begin{tabular}{|c|c|c|c|c|c|}
\hline$i$ & $\left|f_{0}-f_{0}^{a}\right|$ & $\left|g_{0}-g_{0}^{a}\right|$ & $\left|f_{5}-f_{5}^{a}\right|$ & $\left|g_{5}-g_{5}^{a}\right|$ & Wronskian \\
\hline \multicolumn{6}{|c|}{$\nu=5$} \\
\hline 1 & $6.11_{10}(-09)$ & $1.76_{10}(-06)$ & $1.12_{10}(-03)$ & $6.14_{10}(-04)$ & $4.36_{10}(-08)$ \\
\hline 2 & $4.54_{10}(-12)$ & $1.03_{10}(-08)$ & $6.14_{10}(-06)$ & $8.33_{10}(-07)$ & $2.05_{10}(-10)$ \\
\hline 3 & $2.56_{10}(-15)$ & $1.60_{10}(-11)$ & $1.52_{10}(-08)$ & $8.48_{10}(-10)$ & $3.29_{10}(-13)$ \\
\hline 4 & $1.21_{10}(-17)$ & $1.83_{10}(-14)$ & $6.40_{10}(-12)$ & $5.25_{10}(-13)$ & $6.72_{10}(-16)$ \\
\hline 5 & $0.00_{10}(-00)$ & $4.19_{10}(-17)$ & $4.64_{10}(-14)$ & $4.04_{10}(-16)$ & $2.23_{10}(-18)$ \\
\hline \multicolumn{6}{|c|}{$\nu=10$} \\
\hline 1 & $4.24_{10}(-10)$ & $1.14_{10}(-07)$ & $2.90_{10}(-04)$ & $1.63_{10}(-04)$ & $2.76_{10}(-09)$ \\
\hline 2 & $8.50_{10}(-14)$ & $8.17_{10}(-10)$ & $1.84_{10}(-06)$ & $5.76_{10}(-08)$ & $1.64_{10}(-11)$ \\
\hline 3 & $1.45_{10}(-17)$ & $3.20_{10}(-13)$ & $1.10_{10}(-09)$ & $9.06_{10}(-11)$ & $6.64_{10}(-15)$ \\
\hline 4 & $1.08_{10}(-19)$ & $9.89_{10}(-17)$ & $1.74_{10}(-12)$ & $1.25_{10}(-15)$ & $8.07_{10}(-18)$ \\
\hline 5 & $0.00_{10}(-00)$ & $1.88_{10}(-19)$ & $1.35_{10}(-15)$ & $6.74_{10}(-17)$ & $3.44_{10}(-19)$ \\
\hline \multicolumn{6}{|c|}{$\nu=25$} \\
\hline 1 & $1.12_{10}(-11)$ & $2.94_{10}(-09)$ & $4.70_{10}(-05)$ & $2.66_{10}(-05)$ & $7.10_{10}(-11)$ \\
\hline 2 & $3.66_{10}(-16)$ & $2.22_{10}(-11)$ & $3.09_{10}(-07)$ & $1.52_{10}(-09)$ & $4.47_{10}(-13)$ \\
\hline 3 & $0.00_{10}(-00)$ & $1.40_{10}(-15)$ & $2.95_{10}(-11)$ & $2.66_{10}(-12)$ & $2.91_{10}(-17)$ \\
\hline 4 & $0.00_{10}(-00)$ & $0.00_{10}(-00)$ & $5.63_{10}(-14)$ & $7.25_{10}(-18)$ & $1.75_{10}(-19)$ \\
\hline 5 & $0.00_{10}(-00)$ & $0.00_{10}(-00)$ & $6.45_{10}(-18)$ & $3.40_{10}(-19)$ & $1.76_{10}(-19)$ \\
\hline
\end{tabular}


nel of the Lebedev transform. The same functions $A_{\nu}, B_{\nu}, C_{\nu}, D_{\nu}$ can be used for this case.

We have described a method for handling the coefficients and auxiliary functions when the properties of these quantities can be derived from the differential equation of the functions to be approximated. The same or similar Airy-type expansions can be obtained when starting from integral representations in which two saddle points are close together (Wong 2001, Chapter 7). For the coefficients in the expansions the same difficulties may arise in numerical algorithms and the method for deriving Maclaurin expansions of the coefficients is quite different compared with the methods described earlier in this section. For details on the case of integrals we refer to (Vidunas and Temme 2002).

\section{The inversion of cumulative distribution functions}

The inversion of cumulative distribution functions is an important topic in statistics, probability theory and econometrics, in particular for computing percentage points of chi-square, $F$, and Student's $t$-distributions. In the tails of these distributions the numerical inversion is not very easy, and for the standard distributions asymptotic formulas are available.

Here we use the uniform asymptotic expansions of the incomplete gamma functions (see Section 5.2) for inverting these functions for large values of $a$. We start with the relatively simple problem of inverting the complementary error function, again by using asymptotic methods. Finally we describe a different method, giving a high-order Newton-like iteration method.

\subsection{Asymptotic inversion of the complementary error function}

We recall the definition of the complementary error function (here we use real arguments)

$$
\operatorname{erfc} x=\frac{2}{\sqrt{\pi}} \int_{x}^{\infty} e^{-t^{2}} \mathrm{~d} t,
$$

and the asymptotic expansion

$$
\operatorname{erfc} x \sim \frac{e^{-x^{2}}}{x \sqrt{\pi}} \sum_{k=0}^{\infty} \frac{(-1)^{k}(2 k) !}{k !(2 x)^{2 k}}=\frac{e^{-x^{2}}}{x \sqrt{\pi}}\left(1-\frac{1}{2} x^{-2}+\frac{3}{4} x^{-4}-\frac{15}{8} x^{-6}+\cdots\right),
$$

as $x \rightarrow \infty$.

We derive an asymptotic expansion for the inverse $x(y)$ of the function $y(x)=\operatorname{erfc} x$ for small positive values of $y$.

Let $t, \alpha$ and $\beta$ be defined by

$$
t=\frac{2}{\pi y^{2}}, \quad \alpha=\frac{1}{\ln t}, \quad \beta=\ln (\ln t) .
$$


Then we have the expansion

$$
x(y) \sim \frac{1}{\sqrt{2 \alpha}}\left(1+x_{1} \alpha+x_{2} \alpha^{2}+x_{3} \alpha^{3}+x_{4} \alpha^{4}+\cdots\right) .
$$

The first coefficients $x_{k}$ are given by

$$
\begin{aligned}
& x_{1}=-\frac{1}{2} \beta, \\
& x_{2}=-\frac{1}{8}\left(\beta^{2}-4 \beta+8\right), \\
& x_{3}=-\frac{1}{16}\left(\beta^{3}-8 \beta^{2}+32 \beta-56\right), \\
& x_{4}=-\frac{1}{384}\left(15 \beta^{4}-184 \beta^{3}+1152 \beta^{2}-4128 \beta+7040\right), \\
& x_{5}=-\frac{1}{768}\left(21 \beta^{5}-352 \beta^{4}+3056 \beta^{3}-16752 \beta^{2}+57536 \beta-97984\right) .
\end{aligned}
$$

We explain how these coefficients can be obtained. The equation $y=$ $\operatorname{erfc} x$, with $y$ small, will be solved for $x$ by using the asymptotic expansion in (6.2). We square the equation, and write

$$
\frac{1}{2} \pi y^{2}=\frac{e^{-\xi}}{\xi} S^{2}(\xi)
$$

where $\xi=2 x^{2}$ and $S(\xi)$ denotes the function that has the power series in (6.2) as its asymptotic expansion with $2 x^{2}$ replaced by $\xi$.

We can rewrite (6.6) in the form

$$
\xi e^{\xi}=t S^{2}(\xi)
$$

where $t$ is defined in (6.3). We solve this equation for $\xi$, with $t$ large.

We observe that this equation has been discussed in detail in (de Bruijn 1981, Section 2.4) for the case that $S(\xi)=1$. We can apply the same method for constructing an asymptotic expansion of the equation in (6.7). We write

$$
\xi=\ln t-\ln (\ln t)+\eta,
$$

and find that $\eta$ satisfies the relation

$$
(\ln t-\ln (\ln t)+\eta) e^{\eta}=\ln t S^{2}(\ln t-\ln (\ln t)+\eta) .
$$

The quantity $\eta$ can be expanded in the form

$$
\eta=\eta_{1} \alpha+\eta_{2} \alpha^{2}+\eta_{3} \alpha^{3}+\cdots,
$$

where $\alpha$ is defined in (6.3). By using a few terms in the expansion of $S(\xi)$ we find

$$
\eta_{1}=\beta-2, \quad \eta_{2}=\frac{1}{2} \beta^{2}-3 \beta+7,
$$

where $\beta$ is defined in (6.3). 
Table 6.7. Solutions $x(y)$ of the equation $y=\operatorname{erfc} x$ by using (6.4).

\begin{tabular}{||c|c|c|c|c||}
\hline$y$ & $x_{0}|| \operatorname{erfc}\left(x_{0}\right) / y-1 \mid$ & $x_{1}$ & $\left|x_{0} / x_{1}-1\right|$ \\
\hline $10^{-02}$ & 1.820630554 & $0.31_{10}(-02)$ & 1.821591563 & $0.53_{10}(-03)$ \\
$10^{-03}$ & 2.326648925 & $0.51_{10}(-03)$ & 2.326782380 & $0.57_{10}(-04)$ \\
$10^{-04}$ & 2.751038248 & $0.15_{10}(-03)$ & 2.751070914 & $0.12_{10}(-04)$ \\
$10^{-05}$ & 3.123404718 & $0.56_{10}(-04)$ & 3.123415612 & $0.35_{10}(-05)$ \\
$10^{-06}$ & 3.458907270 & $0.25_{10}(-04)$ & 3.458911685 & $0.13_{10}(-05)$ \\
$10^{-07}$ & 3.766560973 & $0.13_{10}(-04)$ & 3.766563021 & $0.54_{10}(-06)$ \\
$10^{-08}$ & 4.052236421 & $0.69_{10}(-05)$ & 4.052237469 & $0.26_{10}(-06)$ \\
$10^{-09}$ & 4.320004932 & $0.41_{10}(-05)$ & 4.320005509 & $0.14_{10}(-06)$ \\
$10^{-10}$ & 4.572824704 & $0.25_{10}(-05)$ & 4.572825039 & $0.74_{10}(-07)$
\end{tabular}

The expansion for $\eta$ gives an expansion for $\xi$ (see (6.8)), and by using $x=\sqrt{\xi / 2}$ we obtain the expansion given in (6.4).

In Table 6.7 we give values of the approximation $x_{0}$ of the solution of the equation $y=\operatorname{erfc} x$ for several values of $y$. We have used the asymptotic expansion (6.4) with the terms up to and including $x_{4}$. The values $x_{1}$ are obtained by using one Newton step. We also give relative errors. The computations are done in Maple with Digits $=10$.

For other methods of the inversion of the error functions we refer to (Strecok 1968), where coefficients of the Maclaurin expansion of $x(y)$, the inverse of $y=\operatorname{erf} x$, are given, with Chebyshev coefficients for an expansion on the $y$-interval $[-0.8,0.8]$. For small values of $y$ (not smaller than $10^{-300}$ ) high-precision coefficients of Chebyshev expansions are given for the numerical evaluation of the inverse of $y=\operatorname{erfc} x$. For rational Chebyshev (near-minimax) approximations for the inverse of the complementary error function $y=\operatorname{erfc} x$ we refer to (Blair, Edwards and Johnson 1976), where $y$-values are considered in the $y$-interval $\left[10^{-10000}, 1\right]$, with relative errors ranging down to $10^{-23}$. An asymptotic formula for the region $y \rightarrow 0$ is also given.

Remark 6.1. We can use similar asymptotic inversion methods for finding complex zeros of the complementary error function; see (Gil et al. 2007). The present case is simpler because we only want to find one real solution with the inversion method.

Remark 6.2. The solution of the equation $\xi e^{\xi}=t$ (see (6.7)) can be expressed in terms of Lambert's $W$-function: $\xi=W(t)$. 


\subsection{Asymptotic inversion of incomplete gamma functions}

We solve the equations

$$
P(a, x)=p, \quad Q(a, x)=q, \quad 0 \leq p \leq 1, \quad 0 \leq q \leq 1,
$$

where $P(a, x)$ and $Q(a, x)$ are the incomplete gamma functions introduced in Section 5.1. We invert the equations for $x$, with $a$ as a large positive parameter. This problem is of importance in probability theory and mathematical statistics. Several approaches are available in the (statistical) literature, where often a first approximation of $x$ is constructed, based on asymptotic expansions, but this first approximation is not always reliable. Higher approximations may be obtained by numerical inversion techniques, which require evaluation of the incomplete gamma functions. This may be rather time-consuming, especially when $a$ is large.

In the present method we also use an asymptotic result. The approximation is quite accurate, especially when $a$ is large. It follows from numerical results, however, that a three-term asymptotic expansion already gives an accuracy of 4 significant digits for $a=2$, uniformly with respect to $p, q \in[0,1]$.

The method is rather general. In a later section we mention application of the same method on a wider class of cumulative distribution functions.

The approximations are obtained by using the uniform asymptotic expansions of the incomplete gamma functions given in Section 5.1, in which the complementary error function is the dominant term. The inversion problem is started by inverting this error function term.

For more details we refer to (Temme 1992a); for the asymptotic inversion of the incomplete beta function, see (Temme 1992b).

\subsection{The asymptotic inversion method}

We perform the inversion of the equations (6.12) with respect to the parameter $\eta$, by using the representations (5.14), with $z$ replaced by $x$ throughout, and large positive values of $a$. Afterwards we have to compute $\lambda$ and $x$ from the relation for $\eta$ in (5.15) and $\lambda=x / a$. We concentrate on the second equation in (6.12). Let us rewrite the inversion problem in the form

$$
\frac{1}{2} \operatorname{erfc}(\eta \sqrt{a / 2})+R_{a}(\eta)=q, \quad q \in[0,1],
$$

which is equivalent to the second equation in (6.12), and we denote the solution of the above equation by $\eta(q, a)$.

To start the procedure we consider $R_{a}(\eta)$ in (6.13) as a perturbation, and we define the number $\eta_{0}=\eta_{0}(q, a)$ as the real number that satisfies the equation

$$
\frac{1}{2} \operatorname{erfc}\left(\eta_{0} \sqrt{a / 2}\right)=q
$$


Known values are

$$
\eta_{0}(0, a)=+\infty, \quad \eta_{0}\left(\frac{1}{2}, a\right)=0, \quad \eta_{0}(1, a)=-\infty .
$$

We note the symmetry $\eta_{0}(q, a)=-\eta_{0}(p, a)$. Computation of $\eta_{0}$ requires an inversion of the error function, but this problem has been satisfactorily solved in the literature; see (Blair et al. 1976) and (Strecok 1968). The value $\eta$ defined by (6.13) is, for large values of $a$, approximated by the value $\eta_{0}$. We write

$$
\eta(q, a)=\eta_{0}(q, a)+\varepsilon\left(\eta_{0}, a\right),
$$

and we try to determine the function $\varepsilon$. It appears that we can expand this quantity in the form

$$
\varepsilon\left(\eta_{0}, a\right) \sim \frac{\varepsilon_{1}}{a}+\frac{\varepsilon_{2}}{a^{2}}+\frac{\varepsilon_{3}}{a^{3}}+\cdots,
$$

as $a \rightarrow \infty$. The coefficients $\varepsilon_{i}$ will be written explicitly as functions of $\eta_{0}$.

We first remark that (6.13) yields the relation

$$
\frac{\mathrm{d} q}{\mathrm{~d} \eta}=\frac{\mathrm{d}}{\mathrm{d} \eta} Q(a, x)=\frac{\mathrm{d}}{\mathrm{d} x} Q(a, x) \frac{\mathrm{d} x}{\mathrm{~d} \eta} .
$$

Using the definition of $Q(a, x)$ and the relation for $\eta$ in (5.15), we obtain, after straightforward calculations,

$$
\frac{\mathrm{d} q}{\mathrm{~d} \eta}=-\frac{1}{\Gamma^{*}(a)} \sqrt{\frac{a}{2 \pi}} f(\eta) e^{-\frac{1}{2} a \eta^{2}},
$$

where $\Gamma^{*}(a)$ is defined in $(5.25)$, and

$$
f(\eta)=\frac{\eta}{\lambda-1},
$$

the relation between $\eta$ and $\lambda$ being given in (5.15). For small values of $\eta$ we can expand

$$
f(\eta)=1-\frac{1}{3} \eta+\frac{1}{12} \eta^{2}+\cdots .
$$

From (6.14) we obtain

$$
\frac{\mathrm{d} q}{\mathrm{~d} \eta_{0}}=-\sqrt{\frac{a}{2 \pi}} e^{-\frac{1}{2} a \eta_{0}^{2}} .
$$

Upon dividing (6.19) and (6.22) we eliminate $q$, although it is still present in $\eta_{0}$. So we obtain

$$
\frac{\mathrm{d} \eta}{\mathrm{d} \eta_{0}}=\frac{\Gamma^{*}(a)}{f(\eta)} e^{\frac{1}{2} a\left(\eta^{2}-\eta_{0}^{2}\right)}, \quad-\infty<\eta_{0}<\infty .
$$


Substitution of (6.16) gives the differential equation

$$
f\left(\eta_{0}+\varepsilon\right)\left[1+\frac{\mathrm{d} \varepsilon}{\mathrm{d} \eta_{0}}\right]=\Gamma^{*}(a) e^{a \varepsilon\left(\eta_{0}+\frac{1}{2} \varepsilon\right)},
$$

a relation between $\varepsilon$ and $\eta_{0}$, with $a$ as (large) parameter.

It is convenient to write $\eta$ in place of $\eta_{0}$. That is, we try to find the function $\varepsilon=\varepsilon(\eta, a)$ that satisfies the equation

$$
f(\eta+\varepsilon)\left[1+\frac{\mathrm{d} \varepsilon}{\mathrm{d} \eta}\right]=\Gamma^{*}(a) e^{a \varepsilon\left(\eta+\frac{1}{2} \varepsilon\right)} .
$$

When we have obtained the solution $\varepsilon(\eta, a)$ (in fact we find an approximation of the form (6.17)), we write it as $\varepsilon\left(\eta_{0}, a\right)$ and the final value of $\eta$ follows from (6.16). The parameters $\lambda$ and $x$ of the incomplete gamma function then follow from inversion of the relation for $\eta$ in (5.15).

\subsection{Determination of the coefficients $\varepsilon_{i}$}

For large values of $a$ we have $\Gamma^{*}(a)=1+\mathcal{O}\left(a^{-1}\right)$; see (5.26). Comparing dominant terms in (6.25), we infer that the first coefficient $\varepsilon_{1}$ in (6.17) is defined by

$$
f(\eta)=e^{\eta \varepsilon_{1}}
$$

giving

$$
\varepsilon_{1}=\frac{1}{\eta} \ln f(\eta)
$$

It is not difficult to verify that $f$ is positive on $\mathbb{R}, f(0)=1$, and that $f$ is analytic in a neighbourhood of $\eta=0$. It follows that $\varepsilon_{1}=\varepsilon_{1}(\eta)$ is an analytic function on $\mathbb{R}$. For small values of $\eta$ we have, using (6.21),

$$
\varepsilon_{1}=-\frac{1}{3}+\frac{1}{36} \eta+\frac{1}{1620} \eta^{2}+\cdots .
$$

The function $\varepsilon_{1}(\eta)$ is non-vanishing on $\mathbb{R}$ (and hence negative). To show this, consider the equation $f^{2}(\eta)=1$. From (6.20) and the relation for $\eta$ in (5.15), it follows that the corresponding $\lambda$-value should satisfy

$$
-\ln \lambda=(\lambda-1)(2 \lambda-3) .
$$

This equation has only one real solution $\lambda=1$, which gives $\eta=0$. However, for this value $\varepsilon_{1}$ equals $-\frac{1}{3}$.

Further coefficients in (6.17) are obtained by using standard perturbation methods. We need the expansion of $\Gamma^{*}(a)$ given in (5.26), and

$$
f(\eta+\varepsilon)=f(\eta)+\varepsilon f^{\prime}(\eta)+\frac{1}{2} \varepsilon^{2} f^{\prime \prime}(\eta)+\cdots,
$$

in which (6.17) is substituted to obtain an expansion in powers of $a^{-1}$. 
Putting all this into (6.25), we find by comparing terms with equal powers of $a^{-1}$

$$
\begin{gathered}
\varepsilon_{2}=\frac{1}{12 \eta f}\left(12 f \varepsilon_{1}^{\prime}+12 f^{\prime} \varepsilon_{1}-f-6 f \varepsilon_{1}^{2}\right) ; \\
\varepsilon_{3}=\frac{1}{288 \eta f}\left(288 f \varepsilon_{2}^{\prime}+288 f^{\prime} \varepsilon_{1} \varepsilon_{1}^{\prime}-24 f \varepsilon_{1}^{\prime}+288 f^{\prime} \varepsilon_{2}+144 f^{\prime \prime} \varepsilon_{1}^{2}\right. \\
\left.+f-288 f \varepsilon_{1} \varepsilon_{2}-144 f \varepsilon_{2}^{2} \eta^{2}-144 f \varepsilon_{2} \eta \varepsilon_{1}^{2}-36 f \varepsilon_{1}^{4}-24 f^{\prime} \varepsilon_{1}\right) .
\end{gathered}
$$

The derivatives $f^{\prime}$ and $\varepsilon_{j}^{\prime}$ are with respect to $\eta$, and evaluated at $\eta$. It will be obvious that the complexity for obtaining higher-order terms is considerable. The terms shown so far have been obtained by symbolic manipulation. For numerical evaluations it is very convenient to have representations free of derivatives.

The derivatives of $f$ can be eliminated by using

$$
\begin{gathered}
f^{\prime}=f\left(1-f^{2}-f \eta\right) / \eta \\
f^{\prime \prime}=f^{2}\left(-3 \eta-3 f+3 f^{3}+5 f^{2} \eta+2 \eta^{2} f\right) / \eta^{2} .
\end{gathered}
$$

The first relation easily follows from (6.20) and the relation between $\eta$ and $\lambda$. Using these relations in $\varepsilon_{i}$, and eliminating the derivatives of previous $\varepsilon_{j}$, it follows that we can write $\eta^{2 j-1} \varepsilon_{j}$ as a polynomial in $\eta, f, \varepsilon_{1}$. We have

$$
\begin{aligned}
& 12 \eta^{3} \varepsilon_{2}=+12-12 f^{2}-12 f \eta-12 f^{2} \eta \varepsilon_{1}-12 f \eta^{2} \varepsilon_{1}-\eta^{2}-6 \eta^{2} \varepsilon_{1}^{2}, \\
& 12 \eta^{5} \varepsilon_{3}=-30+12 f^{2} \eta \varepsilon_{1}+12 f \eta^{2} \varepsilon_{1}+24 f^{2} \eta^{3} \varepsilon_{1}+6 \varepsilon_{1}^{3} \eta^{3}-12 f^{2} \\
& \quad+31 f^{2} \eta^{2}+72 f^{3} \eta+42 f^{4}+18 f^{3} \eta^{3} \varepsilon_{1}^{2}+6 f^{2} \eta^{4} \varepsilon_{1}^{2}+36 f^{4} \eta \varepsilon_{1}+60 f^{3} \eta^{2} \varepsilon_{1} \\
& \quad+12 \varepsilon_{1}^{2} \eta^{3} f+12 \varepsilon_{1}^{2} \eta^{2} f^{2}-12 \eta \varepsilon_{1}+\eta^{3} \varepsilon_{1}+f \eta^{3}-12 f \eta+12 \varepsilon_{1}^{2} \eta^{2} f^{4}
\end{aligned}
$$

The coefficients $\varepsilon_{1}, \ldots, \varepsilon_{3}$ are bounded on $\mathbb{R}$. To show this one needs

$$
f(\eta) \sim-\eta, \quad \eta \rightarrow-\infty, \quad f(\eta) \sim 2 \eta^{-1}, \quad \eta \rightarrow+\infty,
$$

and the above representations of $\varepsilon_{i}$. We find

$$
\varepsilon_{1} \sim \mp \frac{\ln |\eta|}{\eta}, \quad \varepsilon_{2} \sim-\frac{1}{12 \eta}, \quad \varepsilon_{3} \sim \frac{\varepsilon_{1}}{12 \eta^{2}},
$$

as $\eta \rightarrow \pm \infty$. In deriving the behaviour at $-\infty$ one should take into account that (see (6.20) and the relation between $\eta$ and $\lambda$ )

$$
f(\eta)+\eta=\frac{\lambda \eta}{\lambda-1} \sim-\eta e^{-\frac{1}{2} \eta^{2}}, \quad \eta \rightarrow-\infty .
$$




\subsection{Expansions of the coefficients $\boldsymbol{\varepsilon}_{\boldsymbol{i}}$}

As explained in Section 5.2 the function $f$ is analytic in a strip $|\operatorname{Im} \eta|<\sqrt{2 \pi}$, and it can be expanded in a Taylor series around the origin with radius of convergence $2 \sqrt{\pi}$. All $\varepsilon_{i}$ have similar analytic properties. That is, the coefficients $\varepsilon_{i}$ can be expanded in series as follows:

$$
\varepsilon_{i}=\sum_{n=0}^{\infty} c_{i, n} \eta^{n}, \quad|\eta|<2 \sqrt{\pi}, \quad i=1,2,3, \ldots .
$$

The representations of $\varepsilon_{i}$ given in the previous section are not suitable for numerical computation, first because of the appearance of derivatives of $f$ and $\varepsilon_{i}$, second because of the complexity of the expressions. Therefore, to facilitate numerical evaluations of $\varepsilon_{1}, \ldots, \varepsilon_{3}$ we provide the following Taylor expansions:

$$
\begin{aligned}
\varepsilon_{1}= & -\frac{1}{3}+\frac{1}{36} \eta+\frac{1}{1620} \eta^{2}-\frac{7}{6480} \eta^{3}+\frac{5}{18144} \eta^{4}-\frac{11}{382725} \eta^{5}-\frac{101}{16329600} \eta^{6} \\
& +\frac{37}{9797760} \eta^{7}-\frac{454973}{498845952000} \eta^{8}+\frac{1231}{15913705500} \eta^{9}+\frac{2745493}{84737299046400} \eta^{10} \\
& -\frac{2152217}{127673385840000} \eta^{11}+\frac{119937661}{30505427656704000} \eta^{12} \\
& -\frac{449}{1595917323000} \eta^{13}-\frac{756882301459}{4455179048226816000000} \eta^{14} \\
+ & \frac{12699400547}{153146779782796800000} \eta^{15}-\frac{3224618478943}{170264214140233973760000} \eta^{16}+\cdots \\
\varepsilon_{2}= & -\frac{7}{405}-\frac{7}{2592} \eta+\frac{533}{204120} \eta^{2}-\frac{1579}{2099520} \eta^{3}+\frac{109}{1749600} \eta^{4}+\frac{10217}{251942400} \eta^{5} \\
& -\frac{9281803}{436490208000} \eta^{6}+\frac{919081}{185177664000} \eta^{7}-\frac{100824673}{571976768563200} \eta^{8} \\
& -\frac{311266223}{899963447040000} \eta^{9}+\frac{52310527831}{343186061137920000} \eta^{10}+\cdots \\
\varepsilon_{3}= & \frac{449}{102060}-\frac{63149}{20995200} \eta+\frac{29233}{36741600} \eta^{2}+\frac{346793}{5290790400} \eta^{3} \\
& -\frac{18442139}{130947062400} \eta^{4}+\frac{14408797}{246903552000} \eta^{5}-\frac{1359578327}{129994720128000} \eta^{6} \\
& -\frac{69980826653}{39598391669760000} \eta^{7}+\frac{987512909021}{514779091706880000} \eta^{8}+\cdots
\end{aligned}
$$

\subsection{Numerical examples}

When $p=q=\frac{1}{2}$, the asymptotics are quite simple. Then $\eta_{0}$ of (6.14) equals zero, and from (6.28) and the expansions in the previous section we obtain (6.16) and (6.17) in the form

$$
\eta \sim-\frac{1}{3} a^{-1}-\frac{7}{405} a^{-2}+\frac{449}{102060} a^{-3}+\cdots
$$


Table 6.8. Relative errors $\left|x_{a}-x\right| / x$ and $\left|Q\left(a, x_{a}\right)-q\right| / q$ for several values of $q$ and $a ; x_{a}$ is obtained by asymptotic expansion (6.17), $x$ is a more accurate value.

\begin{tabular}{l|cc|cc|cc}
$q$ & $a=1$ & $a=1$ & $a=5$ & $a=5$ & $a=10$ & $a=10$ \\
\hline 0.0001 & $2.3_{10}(-4)$ & $2.1_{10}(-3)$ & $1.1_{10}(-6)$ & $1.6_{10}(-5)$ & $9.4_{10}(-8)$ & $1.7_{10}(-6)$ \\
0.1 & $6.6_{10}(-4)$ & $1.5_{10}(-3)$ & $2.0_{10}(-6)$ & $9.3_{10}(-6)$ & $1.4_{10}(-7)$ & $8.8_{10}(-7)$ \\
0.3 & $8.7_{10}(-4)$ & $1.0_{10}(-3)$ & $2.3_{10}(-6)$ & $6.4_{10}(-6)$ & $1.6_{10}(-7)$ & $6.0_{10}(-7)$ \\
0.5 & $7.0_{10}(-4)$ & $4.8_{10}(-4)$ & $6.7_{10}(-7)$ & $1.2_{10}(-6)$ & $5.4_{10}(-8)$ & $1.4_{10}(-7)$ \\
0.7 & $4.9_{10}(-4)$ & $1.7_{10}(-4)$ & $2.7_{10}(-6)$ & $2.6_{10}(-6)$ & $1.7_{10}(-7)$ & $2.6_{10}(-7)$ \\
0.9 & $1.9_{10}(-3)$ & $2.0_{10}(-4)$ & $2.5_{10}(-6)$ & $8.8_{10}(-7)$ & $1.8_{10}(-7)$ & $9.3_{10}(-8)$ \\
0.9999 & $5.1_{10}(-3)$ & $5.1_{10}(-7)$ & $3.9_{10}(-6)$ & $1.8_{10}(-9)$ & $6.0_{10}(-8)$ & $4.8_{10}(-11)$ \\
\hline
\end{tabular}

In this case we give an expansion of the requested value $x$. Recall that $x=a \lambda$ and that $\lambda$ can be obtained from the relation between $\eta$ and $\lambda$ in (5.15) with $\eta$ given by (6.44). Inverting

$$
\frac{1}{2} \eta^{2}=\frac{1}{2}(\lambda-1)^{2}-\frac{1}{3}(\lambda-1)^{3}+\frac{1}{4}(\lambda-1)^{4}+\cdots
$$

we obtain

$$
\lambda=1+\eta+\frac{1}{3} \eta^{2}+\frac{1}{36} \eta^{3}-\frac{1}{270} \eta^{4}+\frac{1}{4320} \eta^{5}+\cdots .
$$

Substituting (6.44), we have

$$
x \sim a\left(1-\frac{1}{3} a^{-1}+\frac{8}{405} a^{-2}+\frac{184}{25515} a^{-3}+\cdots\right) .
$$

When $a=1, q=\frac{1}{2}$, the equations in (6.12) reduce to $e^{-x}=\frac{1}{2}$, with solution $x=\ln 2 \doteq 0.693147$, while expansion (6.47) gives $x \sim 0.693631$, an accuracy of 3 digits. When $a=2, q=\frac{1}{2}$, the equations in (6.12) become $(1+x) e^{-x}=\frac{1}{2}$, with solution $x \doteq 1.6783469$; in this case our expansion (6.47) gives $x \sim 1.6783461$, an accuracy of 6 significant digits. This shows that (6.47) is quite accurate for small values of the (large) parameter $a$. Computer experiments show that for other $q$-values the results are of the same kind. See Table 6.8.

In Table 6.8 we give more results of numerical experiments. We have used (6.17) with three terms. The first column under each $a$-value gives the relative accuracy $\left|x_{a}-x\right| / x$, where $x_{a}$ is the result of the asymptotic method, and $x$ is a more accurate value obtained by Newton's method. The second column under each $a$-value gives the relative errors $\left|Q\left(a, x_{a}\right)-q\right| / q$. 


\subsection{Generalizations}

The method described in the previous sections can be applied to other cumulative distribution functions. Consider the function

$$
F_{a}(\eta)=\sqrt{\frac{a}{2 \pi}} \int_{-\infty}^{\eta} e^{-\frac{1}{2} a \zeta^{2}} f(\zeta) \mathrm{d} \zeta
$$

where $a>0$ and $\eta \in \mathbb{R}$. We assume that $f$ is an analytic function in a domain containing the real axis, and that $f$ is positive on $\mathbb{R}$ with the normalization $f(0)=1$. In (Temme 1982) it is shown that several well-known distribution functions can be written in this form, including the incomplete gamma and beta functions. It is also shown that the following representation holds:

$$
F_{a}(\eta)=\frac{1}{2} \operatorname{erfc}(-\eta \sqrt{a / 2}) F_{a}(\infty)+R_{a}(\eta),
$$

where $R_{a}(\eta)$ can be expanded as in (5.16). $F_{a}(\infty)$ is the complete integral, and can be expanded in the form

$$
F_{a}(\infty) \sim \sum_{n=0}^{\infty} \frac{A_{n}}{a^{n}}, \quad \text { as } \quad a \rightarrow \infty, \quad A_{0}=1 .
$$

By dividing both sides of (6.48) by $F_{a}(\infty)$, we obtain a further normalization, which is typical for distribution functions.

The inversion of the equation $F_{a}(\eta) / F_{a}(\infty)=q$, with $q \in[0,1]$ and $a$ a given (large) number, can be performed as in the case of the incomplete gamma functions. As in (6.14), let $\eta_{0}$ be the real number satisfying the equation

$$
\frac{1}{2} \operatorname{erfc}\left(-\eta_{0} \sqrt{a / 2}\right)=q .
$$

Then the requested value $\eta$ is written as in (6.16), and an expansion like (6.17) can be obtained by deriving the differential equation (6.24), with $f$ of $(6.48)$ and $\Gamma^{*}(a)$ replaced with $F_{a}(\infty)$.

\subsection{High-order Newton-like methods}

Special functions usually satisfy a simple ordinary differential equation, and this equation can be used to construct Newton-like methods of high order.

Let $f(z)$ be the function, the zero $\zeta$ of which has to be computed. We put $\zeta=\zeta_{0}+h$, where $\zeta_{0}$ is an approximation of this zero and we assume that we can expand in a neighbourhood of this point

$$
f(\zeta)=f\left(\zeta_{0}+h\right)=f\left(\zeta_{0}\right)+h f_{1}+\frac{1}{2 !} h^{2} f_{2}+\frac{1}{3 !} f_{3}+\cdots,
$$

where $f_{k}$ denotes the $k$ th derivative of $f$ at $\zeta_{0}$. We assume that $f\left(\zeta_{0}\right)$ is small and we expand

$$
h=c_{1} f\left(\zeta_{0}\right)+c_{2} f^{2}\left(\zeta_{0}\right)+c_{3} f^{3}\left(\zeta_{0}\right)+\cdots .
$$


Substituting this expansion into (6.52), using that $f(\zeta)=0$, and comparing equal powers of $f\left(\zeta_{0}\right)$, we find, when $f_{1} \neq 0$,

$$
\begin{array}{ll}
c_{1}=-\frac{1}{f_{1}}, & c_{2}=-\frac{f_{2}}{2 f_{1}^{3}}, \\
c_{3}=\frac{-3 f_{2}^{2}+f_{3}^{3} f_{1}}{6 f_{1}^{5}}, & c_{4}=-\frac{f_{4} f_{1}^{2}+15 f_{2}^{3}-10 f_{2} f_{3} f_{1}}{24 f_{1}^{7}} .
\end{array}
$$

When we neglect in (6.53) the coefficients $c_{k}$ with $k \geq 2$ we obtain Newton's method, with $\zeta \doteq \zeta_{0}-f\left(\zeta_{0}\right) / f^{\prime}\left(\zeta_{0}\right)$.

When $f(z)$ satisfies a simple ordinary differential equation the higher derivatives can be replaced by combinations of lower derivatives.

Example 6.3. (The inversion of the incomplete gamma function) In Section 6.2 we consider the inversion of the equations $P(a, x)=p$, $Q(a, x)=q$, where $0<p<1,0<q<1$, for large positive values of $a$. When $a$ is small the asymptotic methods cannot be applied, although for $a=1$ the results can be used as a first approximation. We take $a \in(0,1], f(x)=P(a, x)-p$ and an initial value $x_{0}>0$. We derive from $f_{1}=f^{\prime}(x)=x^{a-1} e^{-x} / \Gamma(a)$ and (6.54) the values

$$
\begin{aligned}
& c_{1}=-x_{0}^{1-a} e^{x_{0}} \Gamma(a), \\
& c_{2}=\frac{x_{0}+1-a}{2 x_{0}} c_{1}^{2}, \\
& c_{3}=\frac{2 x_{0}^{2}+4 x_{0}(1-a)+2 a^{2}-3 a+1}{6 x_{0}^{2}} c_{1}^{3}, \\
& c_{4}=\frac{6 x_{0}^{3}+18 x_{0}^{2}(1-a)+x_{0}\left(18 a^{2}-29 a+11\right)-6 a^{3}+11 a^{2}-6 a+1}{24 x_{0}^{3}} c_{1}^{4} .
\end{aligned}
$$

For $a=1$ the equation $f(x)=0$ is simple, because $P(1, x)=1-e^{-x}$ and the solution of $f(x)=0$ is $x=-\ln (1-p)$. The values $c_{k}$ are in this case $c_{k}=(-1)^{k} e^{k x_{0}} / k, k=1,2,3, \ldots$, and $h$ of $(6.53)$ becomes

$$
h=\sum_{k=1}^{\infty} c_{k} f^{k}=-\ln \left(1+e^{x_{0}} f\left(x_{0}\right)\right) .
$$

Using this value of $h$ we obtain

$$
x=x_{0}+h=x_{0}-\ln \left(1+e^{x_{0}} f\left(x_{0}\right)\right)=-\ln (1-p),
$$

which gives the exact solution of $f(x)=0$, for any $x_{0}>0$.

For general $a \in(0,1]$ we derive a convenient starting value $x_{0}$. We observe that

$$
P(a, x)=\frac{1}{\Gamma(a)} \int_{0}^{x} t^{a-1} e^{-t} \mathrm{~d} t<\frac{1}{\Gamma(a)} \int_{0}^{x} t^{a-1} \mathrm{~d} t=\frac{x^{a}}{\Gamma(a+1)} .
$$


Hence, the solution $x_{0}$ of the equation $x^{a}=p \Gamma(a+1)$ satisfies $0<x_{0}<x$, where $x$ is the exact solution of $f(x)=0$.

The case $a=\frac{1}{2}$ is of special interest, because $P\left(\frac{1}{2}, x\right)=\operatorname{erf} \sqrt{x}$, the error function; see also Section 6.1. For a numerical example we take $p=0.5$. We have $x_{0}=\pi / 16=0.196349540849362$ and $f\left(x_{0}\right)=\operatorname{erf} \sqrt{x_{0}}-\frac{1}{2}=$ -0.030884051069941 . Using the values values $c_{1}, c_{2}, c_{3}, c_{4}$ from $(6.57)$, we have $h=0.0311185517296367$. This gives the new approximation $x \doteq x_{0}+$ $h=0.227468092579000$ and with this value we have $f(x)=-1.12 \cdots 10^{-7}$. It is easy to iterate and to obtain much higher accuracy.

\section{How to handle series with special functions}

When we have solved the problem of writing good software for a class of special functions for a wide range of the real or complex parameters, some application problems remain unsolved. We consider two examples in which special functions arise in representations of solutions of certain problems from probability theory and mathematical physics. We consider one series with incomplete gamma functions and one with modified Bessel functions.

\subsection{The non-central chi-squared distribution functions}

We start from (Abramowitz and Stegun 1964, Equation 26.4.25) and consider for positive $x, y, \mu$ the non-central chi-squared distribution functions (which are also called non-central gamma distributions)

$$
P_{\mu}(x, y)=e^{-x} \sum_{n=0}^{\infty} \frac{x^{n}}{n !} P(\mu+n, y), \quad Q_{\mu}(x, y)=e^{-x} \sum_{n=0}^{\infty} \frac{x^{n}}{n !} Q(\mu+n, y),
$$

in terms of the incomplete gamma functions, which are related to the standard chi-squared probability functions,

$$
P(a, x)=\frac{1}{\Gamma(a)} \int_{0}^{x} t^{a-1} e^{-t} \mathrm{~d} t, \quad Q(a, x)=\frac{1}{\Gamma(a)} \int_{x}^{\infty} t^{a-1} e^{-t} \mathrm{~d} t .
$$

Because $P(a, x)+Q(a, x)=1$ we also have

$$
P_{\mu}(x, y)+Q_{\mu}(x, y)=1
$$

Integral representations in terms of modified Bessel functions follow from replacing the incomplete gamma functions in (7.1) by their integral representations, and by using

$$
I_{\mu}(z)=\left(\frac{1}{2} z\right)^{\mu} \sum_{n=0}^{\infty} \frac{\left(\frac{1}{2} z\right)^{2 n}}{\Gamma(\mu+n+1) n !},
$$


which gives

$$
\begin{gathered}
P_{\mu}(x, y)=e^{-x} \int_{0}^{y}\left(\frac{t}{x}\right)^{\frac{1}{2}(\mu-1)} e^{-t} I_{\mu-1}(2 \sqrt{x t}) \mathrm{d} t \\
Q_{\mu}(x, y)=e^{-x} \int_{y}^{\infty}\left(\frac{t}{x}\right)^{\frac{1}{2}(\mu-1)} e^{-t} I_{\mu-1}(2 \sqrt{x t}) \mathrm{d} t .
\end{gathered}
$$

The function $Q_{\mu}(x, y)$ plays a role in physics and engineering, for instance in problems on radar communications, where it is called the generalized Marcum $Q$-function. In (Marcum 1960) the function is considered with $\mu=1$. The parameter $\mu$ is related to the degrees of freedom and $y$ to the non-centrality. The recurrence relations of the incomplete gamma functions

$$
P(a+1, x)=P(a, x)-\frac{x^{a} e^{-x}}{\Gamma(a+1)}, \quad Q(a+1, x)=Q(a, x)+\frac{x^{a} e^{-x}}{\Gamma(a+1)},
$$

give the recursions

$$
\begin{aligned}
& P_{\mu+1}(x, y)=P_{\mu}(x, y)-\left(\frac{y}{x}\right)^{\frac{1}{2} \mu} e^{-x} I_{\mu}(2 \sqrt{x y}) \\
& Q_{\mu+1}(x, y)=Q_{\mu}(x, y)+\left(\frac{y}{x}\right)^{\frac{1}{2} \mu} e^{-x} I_{\mu}(2 \sqrt{x y}) .
\end{aligned}
$$

We can eliminate the Bessel function in (7.9) using $I_{\mu-1}(z)=I_{\mu+1}(z)+$ $(2 \mu / z) I_{\mu}(z)$. This gives the homogeneous third-order recurrence relation:

$$
x Q_{\mu+2}(x, y)=(x-\mu) Q_{\mu+1}(x, y)+(y+\mu) Q_{\mu}(x, y)-y Q_{\mu-1}(x, y) .
$$

Because a constant satisfies this equation, it also holds for $P_{\mu}(x, y)$.

By using the integral representation of the modified Bessel function (see (Abramowitz and Stegun 1964, 29.3.81))

$$
\left(\frac{z}{x}\right)^{\frac{1}{2}(\mu-1)} I_{\mu-1}(2 \sqrt{x z})=\frac{1}{2 \pi \mathrm{i}} \int e^{s z+x / s} s^{-\mu} \mathrm{d} s, \quad \mu>0,
$$

where the path of integration may be any vertical line in the half-plane $\operatorname{Re} s>0$. The path may be deformed into a Hankel contour $\mathcal{L}$ shown in Figure 3.2. Substituting the loop integral into the integral in (7.6), we obtain

$$
Q_{\mu}(x, y)=\frac{e^{-x-y}}{2 \pi i} \int_{c-\mathrm{i} \infty}^{c+\mathrm{i} \infty} \frac{e^{x / s+y s}}{(1-s) s^{\mu}} \mathrm{d} s, \quad 0<c<1 .
$$

When we move the vertical line to the right, across the pole at $s=1$, and take into account the residue, we obtain

$$
P_{\mu}(x, y)=\frac{e^{-x-y}}{2 \pi i} \int_{c-\mathrm{i} \infty}^{c+\mathrm{i} \infty} \frac{e^{x / s+y s}}{(1-s) s^{\mu}} \mathrm{d} s, \quad c>1 .
$$


These representations are useful for deriving asymptotic expansions. In problems in radar communications very large values of $\mu, x, y$ are used, say, about 10,000 . Asymptotic analysis shows a transition when $y$ passes the value $x+\mu$. There is a fast transition from 0 to 1 . In fact we have

$$
Q_{\mu}(x, y) \sim\left\{\begin{array}{cc}
1 & \text { if } x+\mu>y, \\
\frac{1}{2} & \text { if } x+\mu=y, \\
0 & \text { if } x+\mu<y,
\end{array}\right.
$$

and complementary behaviour for $P_{\mu}(x, y) 1-Q_{\mu}(x, y)$.

In (Temme 1993, Temme 1996) details can be found on two types of asymptotic approximations, one for $x$ and $y$ large, with $\mu$ fixed, and one for all parameters large. In both approximations the complementary error function is used that describes the transition from 0 to 1 , as shown for $Q_{\mu}(x, y)$ in (7.14).

\section{Numerical aspects}

In applications it is of interest to have available algorithms for $Q_{\mu}(x, y)$ when $0<Q_{\mu}(x, y) \leq \frac{1}{2}($ see $(7.14))$ and for $P_{\mu}(x, y)$ otherwise.

The recurrence relation (7.9) is useful for computing $Q_{\mu}(x, y)$. It is numerically stable in the forward direction, since the right-hand side of (7.9) has positive terms. An algorithm for the modified Bessel function is needed. A point of warning: the recurrence relation for the modified Bessel function should not be used in the forward direction, see Example 4.2.

Observe that the function $P_{\mu}(x, y)$ satisfies the recursion

$$
P_{\mu}(x, y)=P_{\mu+1}(x, y)+\left(\frac{y}{x}\right)^{\mu / 2} e^{-x} I_{\mu}(2 \sqrt{x y}),
$$

which is stable in the backward direction.

In the homogeneous recurrence relation (7.10) Bessel functions do not occur. It is attractive to use this equation for $P_{\mu}(x, y)$ and $Q_{\mu}(x, y)$ in order to avoid the recursion of the Bessel functions. However, one needs to investigate the stability of (7.10) in more detail, and for several combinations of the parameters. We know three linearly independent solutions: $P_{\mu}(x, y)$, $Q_{\mu}(x, y)$ and the constant function (with respect to $\mu$. This indicates that it may be stable for $Q_{\mu}(x, y)$ in the forward direction and for $P_{\mu}(x, y)$ in the backward direction.

For small and moderate values of $x, y, \mu$ the expansions in (7.1) can be used. Both series have positive terms and both series require the evaluation of one incomplete gamma function. The series for $Q_{\mu}(x, y)$ requires the value $Q(\mu, y)$, and the remaining terms follow from the stable recursion in (7.7) for $Q(a, x)$.

The series in (7.1) for $P_{\mu}(x, y)$ requires an initial value of $P(a, x)$. The 
recursion in (7.7) should be used in the backward direction. Let $n_{0}$ be the (smallest) number such that

$$
P_{\mu}(x, y) \doteq e^{-x} \sum_{n=0}^{n_{0}} \frac{x^{n}}{n !} P(\mu+n, y)
$$

within the required relative accuracy. Then as starting value we need to compute $P\left(\mu+n_{0}, y\right)$, and the remaining values follow from the recursion in (7.7) for $P(a, x)$. To estimate $n_{0}$ we use

$$
P(\mu+n, y) \sim \frac{y^{n+\mu} e^{-y}}{\Gamma(\mu+n+1)}, \quad \mu+n \rightarrow \infty .
$$

For obtaining relative accuracy, we need an estimate of $P_{\mu}(x, y)$. One can use the value of the integrand of (7.5) at $t=y$, that is,

$$
P_{\mu}(x, y) \sim\left(\frac{y}{x}\right)^{\frac{1}{2} \mu} e^{-x-y} I_{\mu}(\xi), \quad \xi=2 \sqrt{x y},
$$

and we replace $I_{\mu}(\xi)$ by the dominant part of the uniform asymptotic approximation (Abramowitz and Stegun 1964, Equation 9.7.7). That is, we replace $I_{\mu}(\xi)$ by $e^{\mu \eta}$, where

$$
\eta=\operatorname{coth} \gamma-\gamma, \quad \gamma=\operatorname{arcsinh} \frac{\mu}{\xi} .
$$

Combining these estimates, and using the dominant factor in Stirling's approximation of the gamma function, we infer that the equation

$$
\frac{e^{-x} x^{n} P(\mu+n, y)}{n ! P_{\mu}(x, y)}=\varepsilon
$$

can be replaced by the equation

$$
n \log \frac{n}{e x}+(\mu+n) \log \frac{\mu+n}{e y}+\mu\left(\frac{1}{2} \log (y / x)+\operatorname{coth} \gamma-\gamma\right)+\log \varepsilon=0 .
$$

The left-hand side assumes a minimal value at $n=\frac{1}{2} \xi \exp (-\gamma)$. A Newton process (a safe starting value is $\xi \exp (-\gamma)$ ) gives the desired values of $n$, which is taken as the number $n_{0}$ in (7.16).

Table 7.9 shows the number of terms $n_{0}$ used in the series in (7.16), or a similar series for $Q_{\mu}(x, y)$, for several values of $x$. In all cases $\mu=$ 8192, $y=1.05 \mu$. These numbers are as in (Robertson 1969, Table I). For larger values of the parameters the computation can be based on asymptotic expansions, in particular when $y \sim x+\mu$. For these expansions we refer to (Temme 1993, Temme 1996). 
Table 7.9. The number $n_{0}$ is as in (7.16), or a similar series for $Q_{\mu}(x, y)$; $\mu=8192, y=1.05 \mu$; the relative accuracy is $10^{-10}$.

\begin{tabular}{||r|r|c|c||}
\hline$x / \mu$ & $n_{0}$ & $Q_{\mu}(x, y)$ & $P_{\mu}(x, y)$ \\
\hline 0.01 & 150 & $1.984527803_{10}(-04)$ & $9.998015472_{0}(-01)$ \\
0.03 & 355 & $4.000364970_{0}(-02)$ & $9.599963503_{10}(-01)$ \\
0.05 & 543 & $4.985354536_{10}(-01)$ & $5.014645464_{10}(-01)$ \\
0.07 & 727 & $9.556573418_{10}(-01)$ & $4.434265825_{10}(-02)$ \\
0.09 & 894 & $9.996249724_{10}(-01)$ & $3.750276164_{10}(-04)$ \\
0.11 & 1054 & $9.999997188_{10}(-01)$ & $2.811864384_{10}(-07)$ \\
0.13 & 1207 & $1.000000000_{0}(-00)$ & $1.999694515_{10}(-11)$ \\
\hline
\end{tabular}

\subsection{A series of modified Bessel function}

In the well-known Fourier series (Abramowitz and Stegun 1964, Eq. 9.6.34)

$$
e^{z \cos \theta}=I_{0}(z)+2 \sum_{n=1}^{\infty} I_{n}(z) \cos n \theta
$$

the Bessel coefficients are large when $\operatorname{Re} z$ is large and positive. We have

$$
I_{\nu}(z) \sim \frac{e^{z}}{\sqrt{2 \pi z}}, \quad \operatorname{Re} z \rightarrow+\infty .
$$

When $\cos \theta<0$, the left-hand side in (7.22) is very small when $\operatorname{Re} z \rightarrow$ $+\infty$. Hence a lot of cancellations occur in the series, and from a numerical point of view the summation of the series is a very unstable process. The same happens in the simpler expansion $e^{x}=\sum_{n=0}^{n} x^{n} / n$ ! when $x$ is a large negative number.

In a more general Fourier series

$$
a_{0} I_{0}(x)+2 \sum_{n=1}^{\infty} a_{n} I_{n}(x) \cos n \theta,
$$

where $a_{n}$ is slowly varying when $n$ is large, the same instabilities may occur, and in the numerical evaluation for large positive $x$ and $\cos \theta<0$ of such a series serious problems arise.

We have met such a series when solving a singular perturbation problem inside a circle. The equation is a second order elliptic equation with a small 
parameter multiplying the Laplace operator and reads

$$
\varepsilon\left(\frac{\partial^{2} \Phi}{\partial x^{2}}+\frac{\partial^{2} \Phi}{\partial y^{2}}\right)-\frac{\partial \Phi}{\partial y}=1, \quad x^{2}+y^{2}<1,
$$

with boundary condition

$$
\Phi(\cos \theta, \sin \theta)=0
$$

on the boundary of the circle $r=1$. We use polar coordinates

$$
x=r \cos \theta, \quad y=r \sin \theta .
$$

By separating the variables, using the differential equation of the modified Bessel function, and the series in (7.22) for fitting the boundary value, we obtain

$$
\Phi(x, y)=-y-e^{\omega r \sin \theta} \sum_{n=-\infty}^{\infty} \frac{I_{n}^{\prime}(\omega)}{I_{n}(\omega)} I_{n}(\omega r) \cos n(\theta+\pi / 2),
$$

where $\omega=\frac{1}{2 \varepsilon}$.

When $\varepsilon \stackrel{2 \varepsilon}{\rightarrow} 0$ the second-order elliptic operator in (7.25) reduces (in the limit $\varepsilon=0$ ) to a first-order operator. The solution of the reduced equation cannot satisfy the boundary condition on the whole circle. For small values of $\varepsilon$ the solution behaves like (the first-order operator in (7.25) has the upper hand)

$$
\Phi(x, y) \sim w_{0}(x, y), \quad w_{0}(x, y)=-y-\sqrt{1-x^{2}},
$$

which indeed solves the first-order part of the equation (7.25), and fits the boundary condition at the lower side of the circle, but not at the upper side. There is a sudden change from regular behaviour inside the circle to steep behaviour at the upper side of the circle. At this part of the circle a boundary layer occurs, see Figure 7.7. The boundary layer occurs at the upper part of the circle, and not at the lower part of it. This is because the linear operator in (7.25) has a minus sign.

It is quite easy to solve the singular perturbation problem in the lower part of the disk by using the singular perturbation method ( $c f$. for instance (Eckhaus and de Jager 1966)) When we substitute the formal series

$$
\Phi(x, y) \sim \sum_{n=0}^{\infty} \varepsilon^{n} w_{n}(x, y)
$$

into (7.25) and equate equal powers of $\varepsilon$, we find

$$
\frac{\partial w_{0}(x, y)}{\partial y}=-1, \quad \frac{\partial w_{n}(x, y)}{\partial y}=\Delta w_{n-1}(x, y), \quad n=1,2, \ldots,
$$

and all $w_{n}$ should vanish on the lower part of the unit circle. This gives $w_{0}$ 


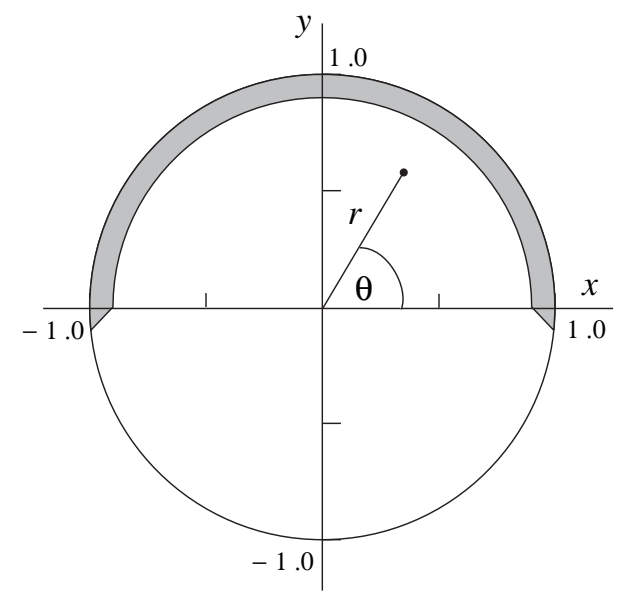

Figure 7.7. Boundary layer inside the circle along the upper boundary $r=1, y>0$ and near the points $( \pm 1,0)$.

as in (7.29) and

$$
w_{n}(x, y)=\int_{-\sqrt{1-x^{2}}}^{y} \Delta w_{n-1}(x, \eta) \mathrm{d} \eta, \quad n=1,2, \ldots, .
$$

It is easily verified that

$$
w_{1}(x, y)=\frac{y+R}{R^{3}}, \quad w_{2}(x, y)=\frac{y+R}{2 R^{7}}\left(3 y+12 y x^{2}+R\right),
$$

where $R=\sqrt{1-x^{2}}$. We observe that these $w_{n}$ become singular at the points $( \pm 1,0)$ and that they do not satisfy the boundary condition $w_{n}=0$ on the upper part of the unit circle. An expansion like (7.30) is called an outer expansion, because it is valid outside the boundary layer.

To satisfy the boundary conditions along the upper part of the unit circle the inner expansion is constructed with so-called boundary layer terms. These functions have the property of being of order $\mathcal{O}\left(\varepsilon^{n}\right)$ for all $n$ everywhere inside the unit circle, except for a small neighbourhood of the upper part of the circle. Following the construction of the boundary layer term given in (Eckhaus and de Jager 1966), we can write in first approximation

$$
\Phi(x, y)=-y-\sqrt{1-x^{2}}+2 \sin \theta e^{-\frac{1}{\varepsilon}(1-r) \sin \theta} \psi(x, y)+z_{0}(x, y, \varepsilon),
$$

where $z_{0}(x, y, \varepsilon)=\mathcal{O}(\varepsilon)$, uniformly inside the unit disk, with the exception of small neighbourhoods of the points $( \pm 1,0)$. The function $\psi$ is a $C^{\infty}$-function, a smoothing factor, on the disk, which equals unity on a neighbourhood of the upper part of the circle, say the domain given by 
$\frac{2}{3}<r \leq 1, y>y_{0}$, where $y_{0}$ is a fixed positive small number, and $\psi$ vanishes in the lower part of the disk.

It is not possible to describe with simple expansions the behaviour of the solution $\Phi(x, y)$ near the points $( \pm 1,0)$. At these points the characteristics of the linear operator touch the boundary of the domain, and it is known that the boundary layers near these points are rather complicated; see (Grasman 1971, Eckhaus and de Jager 1966).

Our interest in this type of simple equations is the following.

(1) Can we find the outer expansion given in (7.30) directly from the exact solution given in (7.28)? Observe that it is quite simple to construct this expansion by using perturbation analysis.

(2) Can we find the inner expansion directly from the exact solution that holds near the upper part of the unit circle? Although it is more complicated than for the outer expansion, the construction of the inner expansion is quite straightforward by using singular perturbation analysis.

(3) Even more challenging, can we find approximations that are valid near the points $( \pm 1,0)$ ?

(4) Can we use the exact solution given in (7.28) to compute the solution in all parts of the unit disk when $\varepsilon \downarrow 0$ with high accuracy? This is of interest for research in numerical methods for singular perturbation problems when numerical algorithms for more general problems need test problems with known exact solution, and where the test problems, although being quite simple, contain essential difficult elements for numerical and asymptotic analysis.

The first two points are considered in a recent paper, Temme (2006). It turns out that the asymptotic analysis can be based on transforming the series by means of the Poisson summation formula (3.50) of which the first term is an integral with respect to the order of the modified Bessel function. By using certain asymptotic approximations of the Bessel functions we can obtain the outer expansion from this first term.

\section{Software for computing special functions}

- For web links to software packages for evaluating special functions we refer to the repository GAMS: Guide to Available Mathematical Software, http://gams.nist.gov/

- In 1994 a complete survey of the available software was published: Lozier and Olver (1994). The latest update of this project appeared in December 2000.

See http://math.nist.gov/mcsd/Reports/2001/nesf/paper.pdf 
- Many interactive systems based on computer algebra, suchas MatlaB, Maple, and Mathematica, have a vast collection of special functions, for symbolic and numerical purposes.

- Mathematical libraries: NETLIB, SPECFUNC, CALGO, SLATEC, CERN, IMSL, NAG. Some are available on a commercial basis, whilst others are freely available (consult the GAMS repository). See also the software published in the journals Computer Physics Communications and Applied Statistics.

- Books, usually with software: (Baker 1992, Moshier 1989, Press, Teukolsky, Vetterling and Flannery 2002, Thompson 1997, Wang and Guo 1989, Zhang and Jin 1996).

\section{Concluding remarks}

This paper deals with special functions that can be represented by an integral, a differential or difference equation, or by other standard ways. We focus on the functions that are useful in applications and we forget about the more exotic functions such as the Fox H-function, the Meijer G-function, and so on, which may be useful in certain analytic descriptions, but are too general to handle from a numerical point of view they.

Also, my choice of the numerical aspects is rather personal, and frequently based on my experience in complex analysis and (uniform) asymptotic approximations. Many other topics are left out, such as continued fractions, best rational approximations, Padé-type approximations, sequence transformations, to name a few, not because they are not of mathematical or computational interest, but simply as a matter of selection for the present paper.

\section{Acknowledgements}

The author acknowledges financial support from the Spanish Ministry of Education and Science (Project MTM2004-01367).

The author thanks SIAM, the Society for Industrial and Applied Mathematics, for allowing this paper to have several topics, parts, and examples in common with the book entitled Numerical Methods for Special Functions written by Amparo Gil, Javier Segura, and the present author, to be published by SIAM in 2007.

\section{REFERENCES}

M. Abramowitz and I. A. Stegun (1964), Handbook of mathematical functions with formulas, graphs, and mathematical tables, Vol. 55 of National Bureau of Standards Applied Mathematics Series, US Printing Office. 
G. E. Andrews, R. Askey and R. Roy (1999), Special functions, Vol. 71 of Encyclopedia of Mathematics and its Applications, Cambridge University Press, Cambridge.

L. Baker (1992), C Mathematical Function Handbook, Programming Tools For Engineers and Scientists, McGraw-Hill, Inc., New York.

W. G. Bickley, L. J. Comrie, J. C. P. Miller, D. H. Sadler and A. J. Thompson (1952), Bessel functions. Part II. Functions of positive integer order, British Association for the Advancement of Science, Mathematical Tables, vol. X, University Press, Cambridge.

J. M. Blair, C. A. Edwards and J. H. Johnson (1976), 'Rational Chebyshev approximations for the inverse of the error function', Math. Comp. 30, 7-68.

M. Blakemore, G. A. Evans and J. Hyslop (1976), 'Comparison of some methods for evaluating infinite range oscillatory integrals', J. Computational Phys. 22(3), 352-376.

W. Bühring (1987), 'An analytic continuation of the hypergeometric series', SIAM J. Math. Anal. 18(3), 884-889.

W. W. Clendenin (1966), 'A method for numerical calculation of Fourier integrals', Numer. Math. 8(5), 422-436.

C. W. Clenshaw (1957), 'The numerical solution of linear differential equations in Chebyshev series', Proc. Cambridge Philos. Soc. 53(1), 134-149.

P. J. Davis and P. Rabinowitz (1984), Methods of numerical integration, Academic Press Inc., Orlando, Fla.

N. G. de Bruijn (1981), Asymptotic methods in analysis, third edn, Dover Publications Inc., New York.

A. Deaño and J. Segura (2006), 'Transitory minimal solutions of hypergeometric recursions and pseudoconvergence of associated continued fractions', Math. Comput. Accepted for publication.

W. Eckhaus and E. M. de Jager (1966), 'Asymptotic solutions of singular perturbation problems for linear differential equations of elliptic type', Arch. Rational Mech. Anal. 23, 26-86.

N. Eggert and J. Lund (1989), 'The trapezoidal rule for analytic functions of rapid decrease', J. Comput. Appl. Math. 27(3), 389-406.

S. Elaydi (2005), An introduction to difference equations, Undergraduate Texts in Mathematics, third edn, Springer, New York.

D. Elliott (1998/99), 'Sigmoidal transformations and the trapezoidal rule', J. Austral. Math. Soc. Ser. B 40((E)), E77-E137 (electronic).

C. Ferreira, J. L. López and E. Pérez Sinusía (2005), 'Incomplete gamma functions for large values of their variables', Adv. in Appl. Math. 34(3), 467-485.

L. N. G. Filon (1928), 'On a quadrature formula for trigonometric integrals', Proc. Roy. Soc. Edinburgh 49(1), 38-47.

R. C. Forrey (1997), 'Computing the hypergeometric function', J. Comput. Phys. 137(1), 79-100.

W. Gautschi (1967), 'Computational aspects of three-term recurrence relations', SIAM Rev. 9(1), 24-82.

W. Gautschi (1977), 'Anomalous convergence of a continued fraction for ratios of Kummer functions', Math. Comp. 31(140), 994-999. 
W. Gautschi (2004), Orthogonal polynomials: computation and approximation, Numerical Mathematics and Scientific Computation, Oxford University Press, New York. Oxford Science Publications.

A. Gil and J. Segura (1997), 'Evaluation of Legendre functions of argument greater than one', Comput. Phys. Comm. 105(2-3), 273-283.

A. Gil and J. Segura (2000), 'Evaluation of toroidal harmonics', Comput. Phys. Comm. 124, 104-122.

A. Gil, J. Segura and N. M. Temme (2006a), 'The ABC of hyper recursions', J. Comput. Appl. Math. 190(1-2), 270-286.

A. Gil, J. Segura and N. M. Temme (2006b), 'Numerically satisfactory solutions of hypergeometric recursions', Math. Comput. Accepted for publication.

A. Gil, J. Segura and N. M. Temme (2007), Numerical methods for special functions, SIAM, Philadelphia, PA. To appear.

E. T. Goodwin (1949), 'The evaluation of integrals of the form $\int_{-\infty}^{\infty} f(x) e^{-x^{2}} \mathrm{~d} x$ ', Proc. Cambridge Philos. Soc. 45(2), 241-245.

J. Grasman (1971), On the birth of boundary layers, Mathematisch Centrum, Amsterdam. No. 36 of Mathematical Centre Tracts.

S. Haber (1977), 'The tanh rule for numerical integration', SIAM J. Numer. Anal. 14(4), 668-685.

A. Iserles (2004), 'On the numerical quadrature of highly-oscillating integrals. I. Fourier transforms', IMA J. Numer. Anal. 24(3), 365-391.

A. Iserles (2005), 'On the numerical quadrature of highly-oscillating integrals. II. Irregular oscillators', IMA J. Numer. Anal. 25(1), 25-44.

A. Iserles and S. P. Nørsett (2005), 'Efficient quadrature of highly oscillatory integrals using derivatives', Proc. R. Soc. Lond. Ser. A Math. Phys. Eng. Sci. 461(2057), 1383-1399.

R. Kreß (1972), 'On the general Hermite cardinal interpolation', Math. Comp. 26, 925-933.

R. Kress and E. Martensen (1970), 'Anwendung der rechteckregel auf die reelle hilbertransformation mit unendlichem intervall', Z. Angew. Math. Mech. $\mathbf{5 0}(1-4)$, T61-T64.

H. Krumhaar (1965), 'Error estimates for Luke's approximation formulas for Bessel and Hankel functions', Z. Angew. Math. Mech. 45, 245-255.

I. M. Longman (1956), 'Note on a method for computing infinite integrals of oscillatory functions', Proc. Cambridge Philos. Soc. 52(4), 764-768.

D. W. Lozier and F. W. J. Olver (1994), Numerical evaluation of special functions, in Mathematics of Computation 1943-1993: a half-century of computational mathematics (Vancouver, BC, 1993), Vol. 48 of Proc. Sympos. Appl. Math., Amer. Math. Soc., Providence, RI, pp. 79-125. Updates are available at http://math.nist.gov/mcsd/Reports/2001/nesf/.

S. K. Lucas and H. A. Stone (1995), 'Evaluating infinite integrals involving Bessel functions of arbitrary order', J. Comput. Appl. Math. 64(3), 217-231.

Y. L. Luke (1969a), The special functions and their approximations I, Vol. 53 of Mathematics in Science and Engineering, Academic Press, New York.

Y. L. Luke (1969b), The special functions and their approximations II, Vol. 53 of Mathematics in Science and Engineering, Academic Press, New York. 
J. N. Lyness (1985), Integrating some infinite oscillating tails, in Proc. International conference on computational and applied mathematics (Leuven, 1984), Vol. $12 / 13$, pp. 109-117.

J. I. Marcum (1960), 'A statistical theory of target detection by pulsed radar', Trans. IRE IT-6, 59-267.

F. Matta and A. Reichel (1971), 'Uniform computation of the error function and other related functions', Math. Comp. 25, 339-344.

M. Mori (1974), 'On the superiority of the trapezoidal rule for the integration of periodic analytic functions', Mem. Numer. Math. 1, 11-19.

S. L. B. Moshier (1989), Methods and programs for mathematical functions, Ellis Horwood Series: Mathematics and its Applications, Ellis Horwood Ltd., Chichester.

A. Murli and M. Rizzardi (1990), 'Algorithm 682: Talbot's method for the Laplace inversion problem', ACM Trans. Math. Software 16(2), 158-168.

F. W. J. Olver (1967), 'Numerical solution of second-order linear difference equations', J. Res. Nat. Bur. Standards Sect. B 71B(2 and 3), 111-129.

F. W. J. Olver (1997), Asymptotics and special functions, AKP Classics, A K Peters Ltd., Wellesley, MA. Reprint of the 1974 original (Academic Press, New York).

R. B. Paris (2002), 'A uniform asymptotic expansion for the incomplete gamma function', J. Comput. Appl. Math. 148(2), 323-339.

T. N. L. Patterson (1976/77), 'On high precision methods for the evaluation of Fourier integrals with finite and infinite limits', Numer. Math. 27(1), 41-52.

W. H. Press, S. A. Teukolsky, W. T. Vetterling and B. P. Flannery (2002), Numerical recipes in $\mathrm{C}++$, Cambridge University Press, Cambridge. The art of scientific computing, Second edition, updated for $\mathrm{C}++$.

S. O. Rice (1973), 'Efficient evaluation of integrals of analytic functions by the trapezoidal rule', Bell System Tech. J. 52, 707-722.

M. Rizzardi (1995), 'A modification of Talbot's method for the simultaneous approximation of several values of the inverse Laplace transform', ACM Trans. Math. Software 21(4), 347-371.

G. Robertson (1969), 'Computation of the noncentral chi-square distribution', Bell. System Tech. J. 48, 201-207.

W. Squire (1976a), 'An efficient iterative method for numerical evaluation of integrals over a semi-infinite range', Internat. J. Numer. Methods Engrg. $\mathbf{1 0}(2), 478-484$.

W. Squire (1976b), 'A quadrature method for finite intervals', Internat. J. Numer. Methods Engrg. 11, 708-712.

A. Strecok (1968), 'On the calculation of the inverse of the error function', Math. Comp. 22, 144-158.

H. Takahasi and M. Mori (1970), 'Error estimation in the numerical integration of analytic functions', Rep. Comput. Centre Univ. Tokyo 3, 41-108.

H. Takahasi and M. Mori (1971), 'Estimation of errors in the numerical quadrature of analytic functions.', Applicable Anal. 1(3), 201-229.

H. Takahasi and M. Mori (1973/74), 'Quadrature formulas obtained by variable transformation', Numer. Math. 21, 206-219. 
A. Talbot (1979), 'The accurate numerical inversion of Laplace transforms', J. Inst. Math. Appl. 23(1), 97-120.

N. M. Temme (1982), 'The uniform asymptotic expansion of a class of integrals related to cumulative distribution functions', SIAM J. Math. Anal. 13(2), 239253.

N. M. Temme (1992a), 'Asymptotic inversion of incomplete gamma functions', Math. Comp. 58(198), 755-764.

N. M. Temme (1992b), 'Asymptotic inversion of the incomplete beta function', $J$. Comput. Appl. Math. 41(1-2), 145-157.

N. M. Temme (1993), 'Asymptotic and numerical aspects of the noncentral chisquare distribution', Comput. Math. Appl. 25(5), 55-63.

N. M. Temme (1996), Special functions, Wiley-Interscience, New York. An introduction to the classical functions of mathematical physics.

N. M. Temme (1997), 'Numerical algorithms for uniform Airy-type asymptotic expansions', Numer. Algorithms 15(2), 207-225.

N. M. Temme (2006), 'Analytical methods for an elliptic singular perturbation problem in a circle', J. Comput. Appl. Math. Accepted for publication.

W. Thompson (1997), An Atlas for Computing Mathematical Functions: An Illustrated Guide for Practitioners, with program in Fortran 90 and Mathematica, Wiley-Interscience, New York.

L. N. Trefethen, J. Weideman and T. Schmelzer (2005), Talbot quadrature and rational approximations, Technical report, Oxford University Computing Laboratory Numerical Analysis Group.

R. Vidunas and N. M. Temme (2002), 'Symbolic evaluation of coefficients in Airytype asymptotic expansions', J. Math. Anal. Appl. 269(1), 317-331.

Z. X. Wang and D. R. Guo (1989), Special functions, World Scientific, Teaneck, NJ. Translated from the Chinese by Guo and X. J. Xia.

J. Weideman (2002), 'Numerical integration of periodic functions: a few examples', Amer. Math. Monthly 109(1), 21-36.

J. Weideman (2005), Optimizing Talbot's contours for the inversion of the Laplace transform, Technical Report NA 05/05, Oxford U. Computing Lab.

E. J. Weniger (1989), 'Nonlinear sequence transformations for the acceleration of convergence and the summation of divergent series', Computer Physics Reports 10(5,6), 189-371.

J. Wimp (1984), Computation with recurrence relations, Applicable Mathematics Series, Pitman (Advanced Publishing Program), Boston, MA.

R. Wong (1982), 'Quadrature formulas for oscillatory integral transforms', Numer. Math. 39(3), 351-360.

R. Wong (2001), Asymptotic approximations of integrals, Vol. 34 of Classics in Applied Mathematics, SIAM, Philadelphia, PA. Corrected reprint of the 1989 original.

R. Wong and H. Li (1992a), 'Asymptotic expansions for second-order linear difference equations', J. Comput. Appl. Math. 41(1-2), 65-94.

R. Wong and H. Li (1992b), 'Asymptotic expansions for second-order linear difference equations. II', Stud. Appl. Math. 87(4), 289-324.

S. Zhang and J. Jin (1996), Computation of special functions, Wiley-Interscience Publication, New York. 